\title{
Cause and Cure - Deterioration in Accuracy of CFD Simulations with Use of High-Aspect-Ratio Triangular/Tetrahedral Grids
}

\author{
Sin-Chung Chang * \\ NASA Glenn Research Center, Cleveland, OH, 44135 \\ Chau-Lyan Chang ${ }^{\dagger}$ \\ NASA Langley Research Center, Hampton, VA, 23681 \\ and \\ Balaji Shankar Venkatachari ${ }^{\ddagger}$ \\ National Institute of Aerospace, Hampton, VA, 23666
}

\begin{abstract}
Traditionally high-aspect ratio triangular/tetrahedral meshes are avoided by CFD researchers in the vicinity of a solid wall, as it is known to reduce the accuracy of gradient computations in those regions. Although for certain complex geometries, the use of highaspect ratio triangular/tetrahedral elements in the vicinity of a solid wall can be replaced by quadrilateral/prismatic elements, ability to use triangular/tetrahedral elements in such regions without any degradation in accuracy can be beneficial from a mesh generation point of view. The benefits also carry over to numerical frameworks such as the spacetime conservation element and solution element (CESE), where simplex elements are the mandatory building blocks. With the requirement of the CESE method in mind, a rigorous mathematical framework that clearly identifies the reason behind the difficulties in use of such high-aspect ratio simplex elements is formulated using two different approaches and presented here. Drawing insights from the analysis, a potential solution to avoid that pitfall is also provided as part of this work. Furthermore, through the use of numerical simulations of practical viscous problems involving high-Reynolds number flows, how the gradient evaluation procedures of the CESE framework can be effectively used to produce accurate and stable results on such high-aspect ratio simplex meshes is also showcased.
\end{abstract}

\section{Introduction}

In the multi-dimensional space-time conservation element and solution element ${ }^{1-6}$ (CESE) method, triangles and tetrahedral mesh elements turn out to be the most natural building blocks for 2D and 3D spatial grids, respectively. As such, the CESE method is naturally compatible with the simplest 2D and 3D unstructured grids and thus can be easily applied to solve problems with complex geometries. However, because (a) accurate solution of a high-Reynolds number flow field near a solid wall requires that the grid intervals along the direction normal to the wall be much finer than those in a direction parallel to the wall and, as such, the use of grid cells with extremely high aspect ratio $\left(10^{3}\right.$ to $\left.10^{6}\right)$ may become mandatory, and (b) unlike quadrilateral/hexahedral grids, it is well-known that accuracy of gradient computations involving triangular/tetrahedral grids tends to deteriorate rapidly as cell aspect ratio increases, the use of triangular/ tetrahedral grid cells near a solid wall has long been deemed impractical by CFD researchers. ${ }^{7}$

In view of (a) the critical role played by triangular / tetrahedral grids in the CESE development, and (b) the importance of accurate resolution of high-Reynolds number flow field near a solid wall, a comprehensive and rigorous mathematical framework that clearly identifies the reasons behind the accuracy deterioration

*Aerospace Engineer, Inlet and Nozzzles Branch.

${ }^{\dagger}$ Aerospace Technologist, Computational AeroSciences Branch, Associate Fellow, AIAA

${ }^{\ddagger}$ Sr.Research Engineer, Senior Member, AIAA 
as described above has been developed for the 2D case involving triangular cells. By avoiding the pitfalls identified by the 2D framework, and its 3D extension, it has been shown numerically that the accuracy deterioration phenomenon identified above can indeed be overcome completely.

\section{A Summary of Key Theoretical Results}

Here, we provide a summary of the key practical results obtained from the current mathematical development. The mathematically-inclined reader is referred to Section III for the more rigorous mathematical treatment and details.

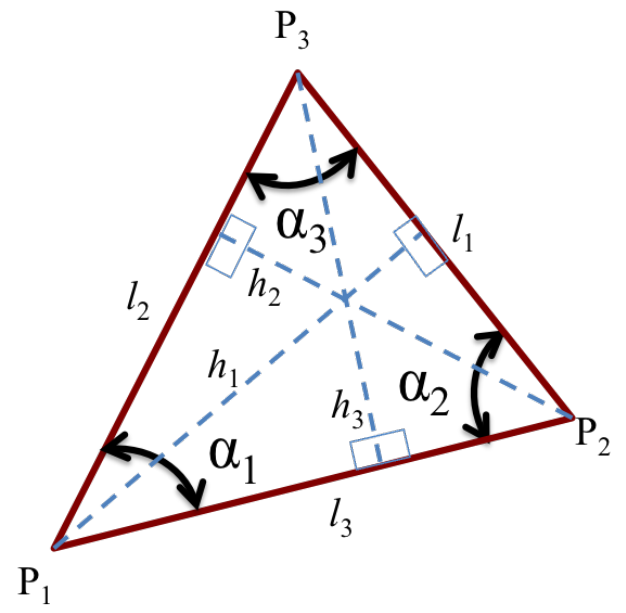

Figure 1. General notations of the vertices, internal angles, lengths of the sides and altitudes of a triangle.

To proceed, let (i) $\Delta P_{1} P_{2} P_{3}$, depicted in Fig.1, be a triangle lying on the $x-y$ plane with its area,

$$
A\left(\Delta P_{1} P_{2} P_{3}\right)>0
$$

and (ii) for each $k=1,2,3$, let $\alpha_{k}$ be the internal angle associated with the vertex, $P_{k}, l_{k}$ be the length of the side facing the vertex $P_{k}$, and $h_{k}$ be the length of the altitude originating from $P_{k}$. Then, (i)

$$
A=\frac{1}{2} l_{k} \cdot h_{k}>0, k=1,2,3
$$

(ii)

$$
l_{k}>0 \text { and } h_{k}>0, k=1,2,3
$$

and (iii)

$$
\pi>\alpha_{1}, \alpha_{2}, \alpha_{3}>0 \text { and } \alpha_{1}+\alpha_{2}+\alpha_{3}=\pi
$$

Because of Eq.(2.3), the aspect ratio $\eta$ of $\Delta P_{1} P_{2} P_{3}$ can be defined as

$$
\eta \stackrel{\text { def }}{=} \max \left\{\frac{l_{1}}{h_{1}}, \frac{l_{2}}{h_{2}}, \frac{l_{3}}{h_{3}}\right\}>0
$$

i.e., $\eta$ is the maximal value of the three positive ratios enclosed within the braces. In turn, by substituting a result of Eq.(2.2), i.e., $h_{k}=\frac{2 A}{l_{k}}, \quad k=1,2,3$ into Eq.(2.5), one has

$$
\eta=\max \left\{\frac{l_{1}}{\left(2 A / l_{1}\right)}, \frac{l_{2}}{\left(2 A / l_{2}\right)}, \frac{l_{3}}{\left(2 A / l_{3}\right)}\right\}=\frac{1}{2 A} \max \left\{\left(l_{1}\right)^{2},\left(l_{2}\right)^{2},\left(l_{3}\right)^{2}\right\}>0
$$

Let $\left(k_{1}, k_{2}, k_{3}\right)$ be a permutation of $(1,2,3)$ such that $l_{k 1} \geq l_{k 2} \geq l_{k 3}$. Then, with the aid of Eq.(2.3), we have

$$
l_{k 1} \geq l_{k 2} \geq l_{k 3}>0
$$


As an example, in the case where $l_{2}>l_{1}>l_{3}, k_{1}=2, k_{2}=1$, and $k_{3}=3$. However in the case where $l_{1}=l_{2}>l_{3}$, one has either (i) $k_{1}=1, k_{2}=2$, and $k_{3}=3$, or (ii) $k_{1}=2, k_{2}=1$ and $k_{3}=3$. With the aid of Eqs.(2.2) and (2.7), Eq.(2.6) now implies that

$$
\eta=\frac{\left(l_{k 1}\right)^{2}}{2 A}=\frac{\left(l_{k 1}\right)^{2}}{l_{k 1} \cdot h_{k 1}}=\frac{l_{k 1}}{h_{k 1}}>0
$$

Note that Eqs.(2.2) and (2.7) imply that $l_{k 1}$ and $h_{k 1}$ respectively, are the lengths of the largest side and the shortest altitude of $\Delta P_{1} P_{2} P_{3}$. According to Eq.(2.8), the ratio of these two lengths is the aspect ratio $\eta$ of $\Delta P_{1} P_{2} P_{3}$.

To recast $\eta$ as a function of the internal angles $\alpha_{1}, \alpha_{2}$, and $\alpha_{3}$, note that, by observing Fig.1 and using Eqs.(2.3) and (2.4), one has the following relations:

$$
\begin{aligned}
h_{1} & =l_{2} \sin \alpha_{3}=l_{3} \sin \alpha_{2}>0 \\
h_{2} & =l_{3} \sin \alpha_{1}=l_{1} \sin \alpha_{3}>0 \\
\text { and } h_{3} & =l_{1} \sin \alpha_{2}=l_{2} \sin \alpha_{1}>0
\end{aligned}
$$

Next, by using Eq.(2.9) and the definition of $k_{1}, k_{2}$, and $k_{3}$, one concludes that

$$
h_{k 1}=l_{k 2} \sin \alpha_{k 3}=l_{k 3} \sin \alpha_{k 2}>0
$$

In turn, by substituting Eq.(2.10) into Eq.(2.8), one has

$$
\eta=\frac{l_{k 1}}{l_{k 2} \sin \alpha_{k 3}}=\frac{l_{k 1}}{l_{k 3} \sin \alpha_{k 2}}>0
$$

Moreover, with the aid of a result of Eq.(2.4), i.e.,

$$
1 \geq \sin \alpha_{k}>0, k=1,2,3
$$

Eq.(2.3) and the law of the sines imply that there exists a constant coefficient $\beta>0$ such that

$$
\frac{l_{k 1}}{\sin \alpha_{k 1}}=\frac{l_{k 2}}{\sin \alpha_{k 2}}=\frac{l_{k 3}}{\sin \alpha_{k 3}}=\beta>0
$$

i.e.,

$$
l_{k 1}=\beta \sin \alpha_{k 1}>0, l_{k 2}=\beta \sin \alpha_{k 2}>0, \text { and } l_{k 3}=\beta \sin \alpha_{k 3}>0
$$

As a result of Eqs.(2.7), (2.12) and (2.14), we have

$$
1 \geq \sin \alpha_{k 1} \geq \sin \alpha_{k 2} \geq \sin \alpha_{k 3}>0
$$

Moreover, by substituting Eq.(2.14) into Eq.(2.11), one can cast $\eta$ as a function of $\alpha_{k 1}, \alpha_{k 2}$ and $\alpha_{k 3}$, i.e.,

$$
\eta=\frac{\sin \alpha_{k 1}}{\sin \alpha_{k 2} \sin \alpha_{k 3}}
$$

As will be shown immediately, $\eta$ can also be expressed as a function involving only $\alpha_{k 2}$ and $\alpha_{k 3}$.

With the aid of Eq.(2.4) and the definition of $k_{1}, k_{2}$, and $k_{3}$, we have

$$
\pi>\alpha_{k 1}, \alpha_{k 2}, \alpha_{k 3}>0 \text { and } \alpha_{k 1}+\alpha_{k 2}+\alpha_{k 3}=\pi
$$

Thus, with the aid of some trigonometric identities, we have

$$
\sin \alpha_{k 1}=\sin \left(\pi-\alpha_{k 2}-\alpha_{k 3}\right) \equiv \sin \left(\alpha_{k 2}+\alpha_{k 3}\right) \equiv\left(\sin \alpha_{k 2}\right)\left(\cos \alpha_{k 3}\right)+\left(\sin \alpha_{k 3}\right)\left(\cos \alpha_{k 2}\right)
$$

Substituting Eq.(2.18) into Eq.(2.16) and using Eq.(2.17), we have

$$
\eta=\cot \alpha_{k 2}+\cot \alpha_{k 3}, \quad 0<\alpha_{k 2}, \alpha_{k 3}<\pi \text { and } \alpha_{k 2}+\alpha_{k 3}<\pi
$$


With the above preliminaries, the following proposition is proved in Appendix A.

Proposition 1: Assuming Eq.(2.17), Eq.(2.15) implies that either

$$
\frac{\pi}{2}>\alpha_{k 1} \geq \alpha_{k 2} \geq \alpha_{k 3}>0 \quad(\text { case Eq.(2.17)) }
$$

or

$$
\pi>\alpha_{k 1} \geq \frac{\pi}{2} \text { and } \frac{\pi}{2} \geq \pi-\alpha_{k 1}>\alpha_{k 2} \geq \alpha_{k 3}>0 \quad \text { (case Eq.(2.17)) }
$$

must be true. In turn, Eqs.(2.16) and (2.19)-(2.21) imply that: (i)

$$
\eta \geq \eta_{\text {min }} \stackrel{\text { def }}{=} \frac{2}{\sqrt{3}}
$$

(ii)

$$
\eta=\eta_{\min } \text { if and only if } \alpha_{1}=\alpha_{2}=\alpha_{3}=\frac{\pi}{3}
$$

$$
\lim _{\alpha_{k 3} \rightarrow 0^{+}} \eta=+\infty
$$

and (iv)

$$
\eta \gg 1 \text { if and only if } \min \left\{\alpha_{1}, \alpha_{2}, \alpha_{3}\right\}=\alpha_{k 3} \ll 1
$$

According to the above proposition, the aspect ratio $\eta$ (i) attains its minimal value $2 / \sqrt{3}$ if and only if $\Delta P_{1} P_{2} P_{3}$ is equilateral, and (ii) attains a very large value if and only if the value of one of the internal angle of $\Delta P_{1} P_{2} P_{3}$ has a very small value.

Next, let the "shape factor" $\gamma$ of $\Delta P_{1} P_{2} P_{3}$ be defined by

$$
\gamma \stackrel{\text { def }}{=} \sin ^{2} \alpha_{1}+\sin ^{2} \alpha_{2}+\sin ^{2} \alpha_{3}
$$

It is shown in section III and Appendix B that (i)

$$
0<\gamma \leq \frac{9}{4}
$$

(ii)

$$
\gamma=\gamma_{\max } \stackrel{\text { def }}{=} \frac{9}{4} \Leftrightarrow \alpha_{1}=\alpha_{2}=\alpha_{3}=\frac{\pi}{3} \Leftrightarrow l_{1}=l_{2}=l_{3}>0
$$

$\gamma \rightarrow 0^{+} \Leftrightarrow \max \left\{\alpha_{1}, \alpha_{2}, \alpha_{3}\right\} \rightarrow \pi^{-}$

and (iv)

$$
\max \left\{\alpha_{1}, \alpha_{2}, \alpha_{3}\right\}=\frac{\pi}{2} \Rightarrow \gamma=2
$$

Hereafter, (i) the symbol " $\Leftrightarrow$ " is used to indicate that the statements given on its left and right sides are equivalent, while the symbol " $\Rightarrow$ " is used to indicate that the statement given on its left side implies that given on the right side; (ii) as an example, $\gamma \rightarrow 0^{+}$states that $\gamma$ approaches 0 from the range $>$ 0 while $\max \left\{\alpha_{1}, \alpha_{2}, \alpha_{3}\right\} \rightarrow \pi^{-}$states that $\max \left\{\alpha_{1}, \alpha_{2}, \alpha_{3}\right\}$ approaches $\pi$ from the range $<\pi$; (iii) as an example, the symbol $\left\{a_{1}, a_{2}, a_{3}\right\}$ denotes a set containing elements $a_{1}, a_{2}$, and $a_{3}$, and, as a result, $\left\{a_{1}, a_{2}, a_{3}\right\}=\left\{b_{1}, b_{2}, b_{3}\right\}$ indicates that the collection of elements $a_{1}, a_{2}$, and $a_{3}$ is identical to that of the elements $b_{1}, b_{2}$, and $b_{3}$; and (iv) as an example, $\left(a_{1}, a_{2}, a_{3}\right) \stackrel{\text { def }}{=}\left\{a_{1},\left\{a_{1}, a_{2}\right\},\left\{a_{1}, a_{2}, a_{3}\right\}\right\}$ so that $\left(a_{1}, a_{2}, a_{3}\right)=$ $\left(b_{1}, b_{2}, b_{3}\right) \Leftrightarrow a_{k}=b_{k}, k=1,2,3$.

According to Eqs.(2.27)-(2.30), (i) $\gamma$ attains its maximal value $9 / 4$ if and only if $\Delta P_{1} P_{2} P_{3}$ is equilateral; (ii) $\gamma$ approaches its minimal value 0 if and only if the largest internal angle of $\Delta P_{1} P_{2} P_{3}$ approaches $\pi^{-}$(and therefore its two other internal angles approach $0^{+}$), e.g., the highly obtuse triangle case depicted in Fig.2(a); and (iii) $\gamma \approx 2$ and thus has a value relatively close to $\gamma_{\max }$ if one of the internal angles of $\Delta P_{1} P_{2} P_{3}$ is close to the right angle even if one of its internal angles approaches $0^{+}$such as the case depicted in Fig.2(b). Note 


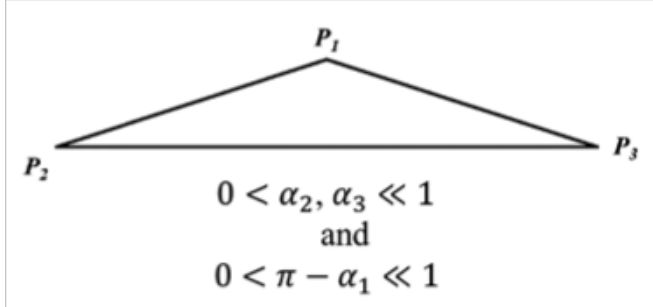

(a)

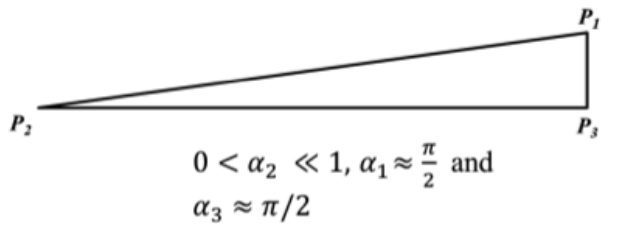

(b)

Figure 2. Representations of two kinds of high-aspect ratio cells: (a) highly obtuse, and (b) nearly right-angle triangle.

that $l_{2} / l_{3}=\sin \alpha_{2} / \sin \alpha_{3} \approx \alpha_{2} / \alpha_{3}$ if $0<\alpha_{2}, \alpha_{3} \ll 1$. Thus, depending on the ratio $\alpha_{2} / \alpha_{3}$, the ratio of the lengths of the two sides $\overline{P_{1} P_{3}}$ and $\overline{P_{1} P_{2}}$ can take any value $>0$ even for the highly obtuse triangle case depicted in Fig.2(a).

Even though the two triangles depicted in Fig.2 have shape factors of vastly different values - one of them approaches the minimal value while another is relatively close to the maximal value of $9 / 4$, both triangles have large aspect ratio. This is due to the fact that both meet the condition that $\min \left\{\alpha_{1}, \alpha_{2}, \alpha_{3}\right\} \ll 1$ which, according to Eq.(2.25) is the necessary and sufficient condition for $\eta \gg 1$.

In Section III, the relation between accuracy deterioration of gradient computation and the shape factor $\gamma$ is established using a procedure briefly described below. First, for each $k=1,2,3$, and at any given time level, let (i) $\phi_{k}$ be the numerical value of a scalar function $\phi$ assigned to the vertex $P_{k}$ of a given triangle $\Delta P_{1} P_{2} P_{3}$ lying on the $x-y$ plane, and (ii) $\left(x_{k}, y_{k}\right)$ be the coordinates of the point $P_{k}$. Second, let $\vec{\nabla} \phi$, the gradient vector of $\phi$ associated with $\Delta P_{1} P_{2} P_{3}$, be evaluated in terms of $\phi_{k}, x_{k}, y_{k}, \quad k=1,2,3$, using the standard linear interpolation procedure defined by Eqs. (3.2), (3.5), (3.6) and (3.21). Third, for each $k=1,2,3$, let $\Delta \phi_{k}$ denote the numerical error of $\phi_{k}$ at the given time level introduced through a timemarching scheme while it is assumed that the coordinates $\left(x_{k}, y_{k}\right), k=1,2,3$, be specified values with no numerical errors. Fourth, with the above assumptions it is shown in Eqs.(3.24), (3.35)-(3.37) and (3.40) that the numerical errors $\epsilon_{1}, \epsilon_{2}$ and $\epsilon_{3}$ of the directional derivatives along the side directions $\overrightarrow{P_{2} P_{3}}, \overrightarrow{P_{3} P_{1}}$, and $\overrightarrow{P_{1} P_{2}}$ of $\Delta P_{1} P_{2} P_{3}$, respectively, are dependent only on $l_{k}$ and $\Delta \phi_{k}, k=1,2,3$, while, according to Eqs. (3.26), (3.40)-(3.43), (3.46), (3.56), (3.57) and (3.66)-(3.69), the numerical errors $\Delta \nu_{x}$ and $\Delta \nu_{y}$ of $\partial \phi / \partial x$ and $\partial \phi / \partial y$, respectively, are functions of $\Delta \phi_{k}, x_{k}$ and $y_{k}, k=1,2,3$ only. Fifth, the error norm for $\epsilon_{1}, \epsilon_{2}$ and $\epsilon_{3}$ is defined as $\sqrt{\left(\epsilon_{1}^{2}+\epsilon_{2}^{2}+\epsilon_{3}^{2}\right) / 3}$ while that for $\Delta \nu_{x}$ and $\Delta \nu_{y}$ are defined as $\sqrt{\left[\left(\Delta \nu_{x}\right)^{2}+\left(\Delta \nu_{y}\right)^{2}\right] / 2}$. Sixth, as shown in Eq.(3.208), the error amplification factor $R$ for gradient computation in turn is defined as the ratio between the error norm for $\Delta \nu_{x}$ and $\Delta \nu_{y}$ and that for $\epsilon_{1}, \epsilon_{2}$ and $\epsilon_{3}$. Seventh, according to Eq.(3.209) and other associated equations given in Section III, it turns out that the factor $R$ is a function of the internal angles $\alpha_{1}, \alpha_{2}$ and $\alpha_{3}$, and the errors $\epsilon_{1}, \epsilon_{2}$ and $\epsilon_{3}$ only. In fact, according to Eqs.(3.167)-(3.170), the $2 \times 2$ real symmetric matrix $H_{1}$ which appears in Eq.(3.209) is a function of $\alpha_{1}, \alpha_{2}$ and $\alpha_{3}$ only, while, according to Eqs.(3.54), (3.108), (3.112), (3.115) and (3.118), the $2 \times 1$ real column matrix $\hat{\psi}_{1}$ which also appears in Eq.(3.209) is a function of $\alpha_{1}, \alpha_{2}, \alpha_{3}, \epsilon_{2}$ and $\epsilon_{3}$ (note: $\epsilon_{1}, \epsilon_{2}, \epsilon_{3}, \alpha_{1}, \alpha_{2}$ and $\alpha_{3}$ are linked by Eq.(3.89), as such, given a set of $\alpha_{1}, \alpha_{2}$ and $\alpha_{3}$, only two of $\epsilon_{1}, \epsilon_{2}$ and $\epsilon_{3}$ are independent parameters). Finally by using Eq.(3.209) and the Rayleigh-Ritz theorem(Ref.[8], p.43), one concludes that for, any combination of $\epsilon_{1}, \epsilon_{2}$ and $\epsilon_{3},(\mathrm{i})$

$$
\sqrt{\sigma_{-}(\gamma)} \leq R \leq \sqrt{\sigma_{+}(\gamma)}, 0<\gamma \leq 9 / 4
$$

where,

$$
\sigma_{ \pm}(\gamma) \stackrel{\text { def }}{=} \frac{3}{4 \gamma}[3 \pm 2 \sqrt{(9 / 4)-\gamma}], 0<\gamma \leq 9 / 4
$$

are the two eigenvalues of $H_{1}$, and (ii) each of the lower and upper bounds $\sqrt{\sigma_{-}(\gamma)}$ and $\sqrt{\sigma_{+}(\gamma)}$ in Eq.(2.31) can be attained by $R$ by some special combinations of $\epsilon_{1}, \epsilon_{2}$ and $\epsilon_{3}$. As such $\sqrt{\sigma_{-}(\gamma)}$ and $\sqrt{\sigma_{+}(\gamma)}$ are the greatest lower bound and the least upper bound of $R$, respectively.

According to Eq.(2.32), both eigenvalues of $H_{1}$ are functions of the shape factor $\gamma$ only, even though $H_{1}$ itself is a complicated matrix function of $\alpha_{1}, \alpha_{2}$ and $\alpha_{3}$ defined by Eqs.(3.167)-(3.170). Also, by using Eqs.(2.27) and (2.32), it is shown in Section III that, as the value of $\gamma$ decreases from its maximal value $9 / 4$ to its minimal limit $0^{+}$, (i) the value of $\sigma_{+}(\gamma)$ increases monotonically from 
1 to $+\infty$; and (ii) the value of $\sigma_{-}(\gamma)$ decreases monotonically from 1 toward the limit value of 1/2. Thus, with the aid of Eqs.(2.28) and (2.29), one has (i)

$$
\begin{array}{r}
\sigma_{-}(\gamma)=1 \Leftrightarrow \sigma_{+}(\gamma)=1 \Leftrightarrow H_{1}=I_{2} \stackrel{\text { def }}{=} \text { the } 2 \times 2 \text { identity matrix } \\
\Leftrightarrow \gamma=\frac{9}{4} \Leftrightarrow \alpha_{1}=\alpha_{2}=\alpha_{3}=\frac{\pi}{3} \Leftrightarrow l_{1}=l_{2}=l_{3}>0
\end{array}
$$

(ii)

$$
\frac{1}{2}<\sigma_{-}(\gamma)<1<\sigma_{+}(\gamma), \quad \text { if } 0<\gamma<\frac{9}{4}
$$

$$
\sigma_{-}(\gamma) \rightarrow\left(\frac{1}{2}\right)^{+} \Leftrightarrow \sigma_{+}(\gamma) \rightarrow+\infty \Leftrightarrow \gamma \rightarrow 0^{+} \Leftrightarrow \max \left\{\alpha_{1}, \alpha_{2}, \alpha_{3}\right\} \rightarrow \pi^{-}
$$

and (iv)

$$
\max \left\{\alpha_{1}, \alpha_{2}, \alpha_{3}\right\}=\frac{\pi}{2} \Rightarrow \gamma=2 \Rightarrow \sqrt{\sigma_{+}(\gamma)}=\sqrt{\frac{3}{2}} \approx 1.225
$$

With the aid of Eqs.(2.33)-(2.36), Eq.(2.31) implies that, (i) the error amplification $R$ for gradient computation has the constant value 1 for any combination of $\epsilon_{1}, \epsilon_{2}$ and $\epsilon_{3}$, if and only if $\Delta P_{1} P_{2} P_{3}$ is equilateral - as such the most accurate gradient computation occurs in the case where the triangular grid is built from equilateral triangles, (ii) for the case in which the grid is built from triangles similar to that depicted in Fig.2(b), the least upper bound of $R$ is only slightly greater than 1 - as such accuracy deterioration of gradient computation is rather slight for this case, and (iii) because $\sqrt{\sigma_{+}(\gamma)}$ approaches $+\infty$ as $\gamma \rightarrow 0^{+}$, accuracy deterioration of gradient computation progressively becomes worse when triangles used in grid construction become more obtuse and it would reach an unacceptable level if these triangles become highly obtuse, similar to the triangle depicted in Fig.2(a). Fortunately, the triangular grids suitable for twodimensional viscous simulations near a solid-wall boundary can always be constructed using triangles similar to that depicted in Fig.2(b). As will be shown in Section IV, for grids containing triangles similar to that depicted in Fig.2(b), the gradient evaluation procedures within the CESE method can be effectively used to improve numerical stability while maintaining numerical accuracy.

Note that, as will be shown in a future paper, the mathematical development presented here for triangular grid can be extended in a straightforward manner to a tetrahedral-grid case, albeit the algebra becomes more complicated. In particular, the square of the error amplification factor $R$ for a tetrahedral-grid case can still be cast into the form given on the extreme right side of Eq.(3.209) with the understanding that $H_{1}$ is a $3 \times 3$ real symmetric and positive-definite matrix [Ref.9, p.250] while $\hat{\psi}_{1}$ is a $3 \times 1$ real column matrix. In fact, it can be shown that, for a regular tetrahedron (i.e., a tetrahedron with all its four faces being equilateral triangles), $H_{1}$ is reduced to the $3 \times 3$ identity matrix, and as such the factor $R=1$ for all possible combinations of the numerical errors associated with the directional derivatives evaluated along all six edge-directions of the tetrahedron. In other words, no accuracy deterioration of gradient computation occurs for a grid built from regular tetrahedrons.

Moreoever, it can be shown that, for a tetrahedron in which three right internal angles share a common vertex (trirectangular tetrahedron), the least upper bound of $R$ is $\sqrt{2} \approx 1.414$, a number slightly larger than 1 . As such accuracy deterioration of gradient computation will be mild for a grid constructed from such tetrahedrons.

\section{A Mathematical Framework for Identifying the Cause and Cure of Accuracy Deterioration Associated with Triangular Grids with High Apsect Ratio}

As a preliminary consider Fig.3(a). Here points $P_{1}, P_{2}$, and $P_{3}$ are the vertices of a triangle lying on the $x-y$ plane with their coordinates, relative to the Cartesian $x-y$ coordinate system shown in Fig.3(b), being $\left(x_{1}, y_{1}\right),\left(x_{2}, y_{2}\right)$, and $\left(x_{3}, y_{3}\right)$ respectively. Let (i)

$$
A\left(\Delta P_{1} P_{2} P_{3}\right) \stackrel{\text { def }}{=} \text { the area of the triangle } \Delta P_{1} P_{2} P_{3}>0
$$




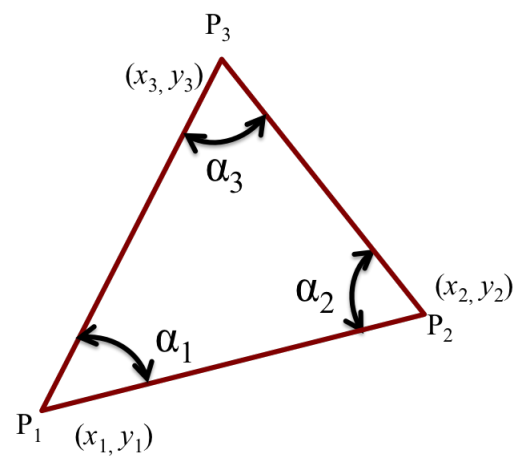

(a)

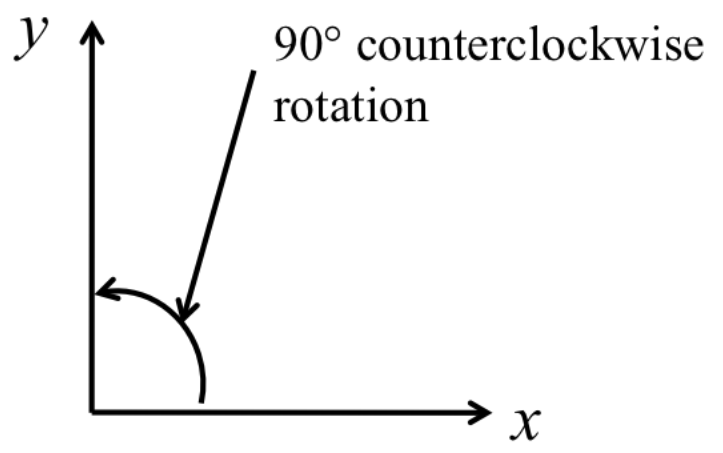

(b)

Figure 3. General representation of (a) the triangle, along with its vertices, coordinates and internal angles; and (b) Cartesian coordinate system.

(ii) the determinant of a square matrix $\mathrm{M}$ be denoted by $|M|$, (iii)

$$
\delta \stackrel{\text { def }}{=}\left|\begin{array}{ll}
x_{2}-x_{1} & y_{2}-y_{1} \\
x_{3}-x_{1} & y_{3}-y_{1}
\end{array}\right|=\left(x_{2}-x_{1}\right)\left(y_{3}-y_{1}\right)-\left(x_{3}-x_{1}\right)\left(y_{2}-y_{1}\right)
$$

and (iv) the rotation from the positive direction of the $x$-axis to that of $y$-axis is in the counterclockwise direction with an angle of $90^{\circ}$. Then it can be shown that: (i)

$$
\delta=2 \cdot A\left(\Delta P_{1} P_{2} P_{3}\right)>0
$$

for the case in which the boundary of $\Delta P_{1} P_{2} P_{3}$ traced in the $P_{1} \rightarrow P_{2} \rightarrow P_{3} \rightarrow P_{1}$ direction forms a counterclockwise loop, and (ii)

$$
\delta=2 \cdot A\left(\Delta P_{1} P_{2} P_{3}\right)<0
$$

for the case in which the boundary of $\Delta P_{1} P_{2} P_{3}$ traced in the $P_{1} \rightarrow P_{2} \rightarrow P_{3} \rightarrow P_{1}$ direction forms a clockwise loop.

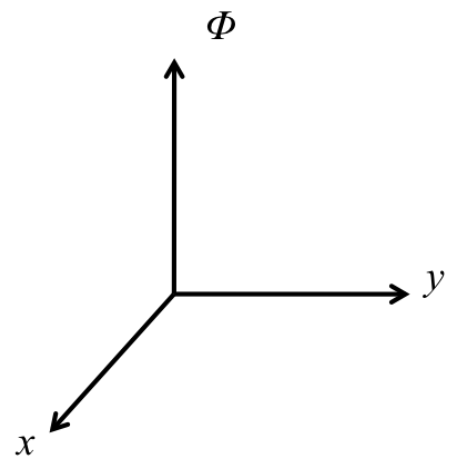

Figure 4. Representation of the $x-y-\phi$ space.

Next, for each $k=1,2,3$, let (i) $\phi_{k}$ be a scalar value assigned to the spatial point $P_{k}$, and (ii) $\hat{P}_{k}$ denote a point in the $x-y-\phi$ space depicted in Fig.4 with the coordinates $\left(x_{k}, y_{k}, \phi_{k}\right)$. In the following, first we will show that points $\hat{P}_{1}, \hat{P}_{2}$, and $\hat{P}_{3}$ lie on the plane in the $x-y-\phi$ space represented by

$$
\phi=a x+b y+c
$$

where, 


$$
a=\frac{\left|\begin{array}{ll}
\phi_{2}-\phi_{1} & y_{2}-y_{1} \\
\phi_{3}-\phi_{1} & y_{3}-y_{1}
\end{array}\right|}{\delta}, \quad b=\frac{\left|\begin{array}{ll}
x_{2}-x_{1} & \phi_{2}-\phi_{1} \\
x_{3}-x_{1} & \phi_{3}-\phi_{1}
\end{array}\right|}{\delta}, \text { and } \quad c=\frac{\left|\begin{array}{lll}
x_{1} & y_{1} & \phi_{1} \\
x_{2} & y_{2} & \phi_{2} \\
x_{3} & y_{3} & \phi_{3}
\end{array}\right|}{\delta}
$$

Proof: By definition, a plane in the $x-y-\phi$ space is represented by

$$
d_{1} x+d_{2} y+d_{3} \phi+d_{4}=0
$$

where $d_{1}, d_{2}, d_{3}$, and $d_{4}$ are real constants with

$$
\left(d_{1}, d_{2}, d_{3}\right) \neq(0,0,0)
$$

i.e., at least one of $d_{1}, d_{2}$, and $d_{3}$ is non-zero.

Next we will show that $\hat{P}_{1}, \hat{P}_{2}$, and $\hat{P}_{3}$ can only lie on a plane represented by Eq.(3.7) with $d_{3} \neq 0$. To prove by contradiction, let $\hat{P}_{1}, \hat{P}_{2}$, and $\hat{P}_{3}$ lie on a plane represented by Eq.(3.7) with $d_{3}=0$. Then

$$
\begin{aligned}
& d_{1} x_{1}+d_{2} y_{1}+d_{4}=0 \\
& d_{1} x_{2}+d_{2} y_{2}+d_{4}=0
\end{aligned}
$$

and

$$
d_{1} x_{3}+d_{2} y_{3}+d_{4}=0
$$

By subtracting Eq.(3.9) from Eqs.(3.10) and (3.11), respectively, one has

$$
\begin{aligned}
\left(x_{2}-x_{1}\right) d_{1}+\left(y_{2}-y_{1}\right) d_{2} & =0 \\
\text { and }\left(x_{3}-x_{1}\right) d_{1}+\left(y_{3}-y_{1}\right) d_{2} & =0
\end{aligned}
$$

On the other hand, according to Eqs.(3.2)-(3.4), we have

$$
\delta \stackrel{\text { def }}{=}\left|\begin{array}{ll}
x_{2}-x_{1} & y_{2}-y_{1} \\
x_{3}-x_{1} & y_{3}-y_{1}
\end{array}\right| \neq 0
$$

In turn, according to elementary algebra, Eqs.(3.12) and (3.13) $\Rightarrow d_{1}=d_{2}=0$. Because Eq.(3.7) does not represent a plane in the $x-y-\phi$ space if $d_{1}=d_{2}=d_{3}=0$, points $\hat{P}_{1}, \hat{P}_{2}$, and $\hat{P}_{3}$ can only lie in a plane represented by Eq. (3.7) with $d_{3} \neq 0$.

Let $d_{3} \neq 0$. Then Eq.(3.7) $\Leftrightarrow$ (i.e., is true if and only if)

$$
\phi=a^{\prime} x+b^{\prime} y+c^{\prime}
$$

where

$$
a^{\prime} \stackrel{\text { def }}{=}-\frac{d_{1}}{d_{3}}, b^{\prime} \stackrel{\text { def }}{=}-\frac{d_{2}}{d_{3}}, \text { and } c^{\prime} \stackrel{\text { def }}{=}-\frac{d_{4}}{d_{3}}
$$

Note that, according to the above derivation of Eq.(3.14), the plane intersecting any three points $\hat{P}_{1}, \hat{P}_{2}$, and $\hat{P}_{3}$ with $A\left(\Delta P_{1} P_{2} P_{3}\right) \neq 0$ (i.e., $P_{1}, P_{2}$, and $P_{3}$ are not collinear points on the $x-y$ plane) must be represented by Eq.(3.14) with the coefficients $a^{\prime}, b^{\prime}$, and $c^{\prime}$ satisfying the conditions

$$
\text { ran } \begin{aligned}
a^{\prime} x_{1}+b^{\prime} y_{1}+c^{\prime} & =\phi_{1} \\
a^{\prime} x_{2}+b^{\prime} y_{2}+c^{\prime} & =\phi_{2} \\
\text { and } \quad a^{\prime} x_{1}+b^{\prime} y_{3}+c^{\prime} & =\phi_{3}
\end{aligned}
$$

Because (i) Eq.(3.16) represents a system of three linear equations for the coefficients $a^{\prime}, b^{\prime}$, and $c^{\prime}$, and (ii) with the aid of Eq.(3.13), we have

$$
\left|\begin{array}{lll}
x_{1} & y_{1} & 1 \\
x_{2} & y_{2} & 1 \\
x_{3} & y_{3} & 1
\end{array}\right| \equiv\left|\begin{array}{ccc}
x_{1} & y_{1} & 1 \\
x_{2}-x_{1} & y_{2}-y_{1} & 0 \\
x_{3}-x_{1} & y_{3}-y_{1} & 0
\end{array}\right| \equiv\left|\begin{array}{cc}
x_{2}-x_{1} & y_{2}-y_{1} \\
x_{3}-x_{1} & y_{3}-y_{1}
\end{array}\right|=\delta \neq 0
$$


according to elementary theory of linear system of equations, the coefficients $a^{\prime}, b^{\prime}$, and $c^{\prime}$ can be uniquely determined in terms of $x_{k}, y_{k}$, and $\phi_{k}, k=1,2,3$. In fact, with the aid of Eqs.(3.6) and (3.17), we have (i)

$$
a^{\prime}=\frac{\left|\begin{array}{lll}
\phi_{1} & y_{1} & 1 \\
\phi_{2} & y_{2} & 1 \\
\phi_{3} & y_{3} & 1
\end{array}\right|}{\delta}=\frac{\left|\begin{array}{ccc}
\phi_{1} & y_{1} & 1 \\
\phi_{2}-\phi_{1} & y_{2}-y_{1} & 0 \\
\phi_{3}-\phi_{1} & y_{3}-y_{1} & 0
\end{array}\right|}{\delta}=\frac{\left|\begin{array}{ll}
\phi_{2}-\phi_{1} & y_{2}-y_{1} \\
\phi_{3}-\phi_{1} & y_{3}-y_{1}
\end{array}\right|}{\delta}=a
$$

(ii)

$$
b^{\prime}=\frac{\left|\begin{array}{lll}
x_{1} & \phi_{1} & 1 \\
x_{2} & \phi_{2} & 1 \\
x_{3} & \phi_{3} & 1
\end{array}\right|}{\delta}=\frac{\left|\begin{array}{ccc}
x_{1} & \phi_{1} & 1 \\
x_{2}-x_{1} & \phi_{2}-\phi_{1} & 0 \\
x_{3}-x_{1} & \phi_{3}-\phi_{1} & 0
\end{array}\right|}{\delta}=\frac{\left|\begin{array}{ll}
x_{2}-x_{1} & \phi_{2}-\phi_{1} \\
x_{3}-x_{1} & \phi_{3}-\phi_{1}
\end{array}\right|}{\delta}=b
$$

and (iii)

$$
c^{\prime}=\frac{\left|\begin{array}{lll}
x_{1} & y_{1} & \phi_{1} \\
x_{2} & y_{2} & \phi_{2} \\
x_{3} & y_{3} & \phi_{3}
\end{array}\right|}{\delta}=c
$$

Thus the plane defined by Eqs.(3.5) and (3.6) is the same plane defined by Eqs.(3.14) and (3.18)-(3.20). QED.

Hereafter let the plane defined by Eq.(3.5) and (3.6) be denoted by $\Gamma$. Then, on $\Gamma$, Eq.(3.5) implies that the gradient vector

$$
\vec{\nabla} \phi \stackrel{\text { def }}{=} \frac{\partial \phi}{\partial x} \overrightarrow{e_{x}}+\frac{\partial \phi}{\partial y} \overrightarrow{e_{y}}=a \overrightarrow{e_{x}}+b \overrightarrow{e_{y}}(\text { on } \Gamma)
$$

where $\overrightarrow{e_{x}}$, and $\overrightarrow{e_{y}}$ are the unit vectors in the $x$ - and $y$ - directions respectively. As such, on $\Gamma$, (a) $\vec{\nabla} \phi$ is a constant vector; and (b) both $\partial \phi / \partial x$ and $\partial \phi / \partial y$ are constant coefficients.

Next, for each $k=1,2,3$, let

$$
\overrightarrow{P_{k}}=x_{k} \overrightarrow{e_{x}}+y_{k} \overrightarrow{e_{y}}, k=1,2,3
$$

i.e., $\overrightarrow{P_{k}}$ is the position vector of point $P_{k}$ on the $x-y$ plane. Also, for each $\left(k_{1}, k_{2}\right)$ with $k_{1} \neq k_{2}$ and $k_{1}, k_{2}=1,2,3$, let

$$
\overrightarrow{P_{k_{1}} P_{k_{2}}} \stackrel{\text { def }}{=} \overrightarrow{P_{k_{2}}}-\overrightarrow{P_{k_{1}}}=\left(x_{k_{2}}-x_{k_{1}}\right) \overrightarrow{e_{x}}+\left(y_{k_{2}}-y_{k_{1}}\right) \overrightarrow{e_{y}}, \quad k_{1} \neq k_{2} \text { and } k_{1}, k_{2}=1,2,3
$$

and

$$
s_{k_{1}, k_{2}} \stackrel{\text { def }}{=}\left|\overrightarrow{P_{k_{1}} P_{k_{2}}}\right|=\sqrt{\left(x_{k_{2}}-x_{k_{1}}\right)^{2}+\left(y_{k_{2}}-y_{k_{1}}\right)^{2}}, k_{1} \neq k_{2} \text { and } k_{1}, k_{2}=1,2,3
$$

(Note here $k_{1}$ and $k_{2}$ are defined independent of Eq.(2.7)). According to Eq.(2.23), $\overrightarrow{P_{k 1} P_{k 2}}$ is the displacement vector joining points $P_{k_{1}}$ and $P_{k_{2}}$ and pointing towards $P_{k_{2}}$ from $P_{k_{1}}$. Also by combining Eqs.(3.1) and (3.24), one concludes that

$$
s_{k_{1}, k_{2}}=s_{k_{2}, k_{1}}>0, k_{1} \neq k_{2} \text { and } k_{1}, k_{2}=1,2,3
$$

As a result, one can define

$$
\vec{e}_{k_{1}, k_{2}} \stackrel{\text { def }}{=} \frac{\overrightarrow{P_{k_{1}} P_{k_{2}}}}{s_{k_{1}, k_{2}}} \quad \text { and } \quad \mu_{k_{1}, k_{2}} \stackrel{\text { def }}{=} \frac{\phi_{k_{2}}-\phi_{k_{1}}}{s_{k_{1}, k_{2}}}, k_{1} \neq k_{2} \text { and } k_{1}, k_{2}=1,2,3
$$

Moreover, Eqs.(3.23)-(3.26) $\Rightarrow$ (a) each $\vec{e}_{k_{1}, k_{2}}$ is an unit vector, i.e.,

$$
\left|\vec{e}_{k_{1}, k_{2}}\right|=1, k_{1} \neq k_{2} \text { and } k_{1}, k_{2}=1,2,3
$$

and (b)

$$
\vec{e}_{k_{2}, k_{1}}=-\vec{e}_{k_{1}, k_{2}} \text { and } \mu_{k_{2}, k_{1}}=-\mu_{k_{1}, k_{2}}, k_{1} \neq k_{2} \text { and } k_{1}, k_{2}=1,2,3
$$


As an example, consider the plane $\Gamma$ which intersects points $\hat{P}_{1}, \hat{P}_{2}$, and $\hat{P}_{3}$. Let $\vec{\nabla} \phi$ be the constant gradient vector on $\Gamma$. Then Eqs. (3.21), and (3.23) $\Rightarrow \vec{\nabla} \phi \bullet \overrightarrow{P_{k_{1}} P_{k_{2}}}=\phi_{k_{2}}-\phi_{k_{1}}$ which, with the aid of Eq. (3.26) in turn $\Rightarrow$

$$
\vec{\nabla} \phi \bullet \vec{e}_{k_{1}, k_{2}}=\mu_{k_{1}, k_{2}}, k_{1} \neq k_{2} \text { and } k_{1}, k_{2}=1,2,3 \quad \text { (on plane } \Gamma \text { ) }
$$

In other words, for each pair of $k_{1}$ and $k_{2}$ with $k_{1} \neq k_{2}$ and $k_{1}, k_{2}=1,2,3, \mu_{k_{1}, k_{2}}$ is the directional derivative of $\phi$ along the $\vec{e}_{k_{1}, k_{2}}$ direction. The value of $\mu_{k_{1}, k_{2}}$ can also be considered as the "slope" of $\overrightarrow{P_{k_{1}} P_{k_{2}}}$ in the $x-y-\phi$ space.

Moreover, Eqs.(3.25), (3.26) and (3.28) imply

$$
\begin{aligned}
& -\mu_{2,1}=\mu_{1,2}=\frac{\phi_{2}-\phi_{1}}{s_{1,2}} \\
& -\mu_{3,2}=\mu_{2,3}=\frac{\phi_{3}-\phi_{2}}{s_{2,3}}
\end{aligned}
$$

and

$$
-\mu_{1,3}=\mu_{3,1}=\frac{\phi_{1}-\phi_{3}}{s_{3,1}}
$$

In turn, Eqs.(3.30)-(3.32) imply

$$
s_{1,2} \cdot \mu_{1,2}+s_{2,3} \cdot \mu_{2,3}+s_{3,1} \cdot \mu_{3,1}=0
$$

and

$$
\mu_{2,1}+\mu_{1,2}=\mu_{3,2}+\mu_{2,3}=\mu_{1,3}+\mu_{3,1}=0
$$

Because the line segments $\overline{P_{1} P_{2}}, \overline{P_{2} P_{3}}$ and $\overline{P_{3} P_{1}}$ are the three sides of $\Delta P_{1} P_{2} P_{3}$, given the scalar values $\phi_{1}, \phi_{2}$ and $\phi_{3}, \mu_{1,2}, \mu_{2,1}, \mu_{3,2}, \mu_{2,3}, \mu_{1,3}$ and $\mu_{3,1}$ represent all possible directional derivatives of $\phi$ which can be evaluated along the spatial directions aligned with the three sides of $\Delta P_{1} P_{2} P_{3}$. Because the six directional derivatives are linked by four independent conditions given in Eqs.(3.33) and (3.34), any one of them can be determined in terms of any two independent directional derivatives among them. In fact, any two of them can be chosen as the independent directional derivatives, as long as they are evaluated along two different sides of $\Delta P_{1} P_{2} P_{3}$ and thus are not linked by one of the three conditions given in Eq. (3.34).

For each $k=1,2,3$, let $\Delta \phi_{k}$ denote the numerical error of $\phi_{k}$ at some given time level introduced through a time-marching procedure. Moreover, (i) let the coordinates $\left(x_{k}, y_{k}\right)$ of point $P_{k}, k=1,2,3$. be given fixed values that do not vary during the time-marching procedure, i.e., the numerical errors $\left(\Delta x_{k}, \Delta y_{k}\right)$ of $\left(x_{k}, y_{k}\right), k=1,2,3$, are assumed to be zero, and (ii) for any pair of $k_{1}$ and $k_{2}$ with $k_{1} \neq k_{2}$ and $k_{1}, k_{2}=1,2,3$, let $\Delta \mu_{k_{1}, k_{2}}$ denote the error of $\mu_{k_{1}, k_{2}}$ introduced as a result of the errors $\Delta \phi_{k_{1}}$ and $\Delta \phi_{k_{2}}$ of $\phi_{k_{1}}$ and $\phi_{k_{2}}$, respectively. Then Eqs.(3.30)-(3.34) $\Rightarrow$

$$
\begin{gathered}
-\Delta \mu_{2,1}=\Delta \mu_{1,2}=\frac{\Delta \phi_{2}-\Delta \phi_{1}}{s_{1,2}} \\
-\Delta \mu_{3,2}=\Delta \mu_{2,3}=\frac{\Delta \phi_{3}-\Delta \phi_{2}}{s_{2,3}} \\
-\Delta \mu_{1,3}=\Delta \mu_{3,1}=\frac{\Delta \phi_{1}-\Delta \phi_{3}}{s_{3,1}} \\
s_{1,2} \cdot \Delta \mu_{1,2}+s_{2,3} \cdot \Delta \mu_{2,3}+s_{3,1} \cdot \Delta \mu_{3,1}=0
\end{gathered}
$$

and

$$
\Delta \mu_{2,1}+\Delta \mu_{1,2}=\Delta \mu_{3,2}+\Delta \mu_{2,3}=\Delta \mu_{1,3}+\Delta \mu_{3,1}=0
$$


Because the six numerical errors $\Delta \mu_{k_{1}, k_{2}}, k_{1} \neq k_{2}$ and $k_{1}, k_{2}=1,2,3$ are linked by four independent conditions given in Eqs.(3.38) and (3.39), any one of them can be determined in terms of any two independent errors among themselves. Any two of these errors can be chosen as independent errors as long as they are not linked by one of the three conditions given in Eq.(3.39), i.e., the numerical errors of any two directional derivatives evaluated along two different sides of $\Delta P_{1} P_{2} P_{3}$ can be chosen as the two independent numerical errors.

To simplify the following developments, let

$$
\epsilon_{1} \stackrel{\text { def }}{=} \Delta \mu_{2,3}, \epsilon_{2} \stackrel{\text { def }}{=} \Delta \mu_{3,1} \text { and } \epsilon_{3} \stackrel{\text { def }}{=} \Delta \mu_{1,2}
$$

and

$$
\vec{e}_{1} \stackrel{\text { def }}{=} \vec{e}_{2,3}, \vec{e}_{2} \stackrel{\text { def }}{=} \vec{e}_{3,1} \text { and } \vec{e}_{3} \stackrel{\text { def }}{=} \vec{e}_{1,2}
$$

Then, with the aid of Eqs.(3.28) and (3.39), Eqs.(3.40) and (3.41) $\Rightarrow$

$$
\Delta \mu_{3,2}=-\epsilon_{1}, \Delta \mu_{1,3}=-\epsilon_{2} \text { and } \Delta \mu_{2,1}=-\epsilon_{3}
$$

and

$$
\vec{e}_{3,2}=-\vec{e}_{1}, \vec{e}_{1,3}=-\vec{e}_{2} \text { and } \vec{e}_{2,1}=-\vec{e}_{3}
$$

At this juncture, note that the integers 1, 2, 3, appear in Eq.(3.40) as component-identification indices. Moreover under the index permutation,

$$
(1,2,3) \rightarrow(2,3,1)
$$

(i.e., the integers 1, 2, and 3, respectively are replaced by 2, 3, and 1), Eq.(3.40) will turn into the form

$$
\epsilon_{2} \stackrel{\text { def }}{=} \Delta \mu_{3,1}, \epsilon_{3} \stackrel{\text { def }}{=} \Delta \mu_{1,2} \text { and } \epsilon_{1} \stackrel{\text { def }}{=} \Delta \mu_{2,3}
$$

i.e., with the exception of them being presented in different order, the definitions given in Eq.(3.40)a are identical to those given in Eq.(3.40). Hereafter, an equation or a set of equations that possesses such a property is said to be invariant under the index permutation of Eq.(3.44). In fact, each similar definition set to be given in Eqs.(3.109)-(3.111) is also invariant under the same index permutation.

Note that, because the numerical errors $\left(\Delta x_{k}, \Delta y_{k}\right)$ of $\left(x_{k}, y_{k}\right), k=1,2,3$ are assumed to be zero in a time-marching procedure, according to Eqs.(3.22)-(3.26), for each pair of $k_{1}$ and $k_{2}$ with $k_{1} \neq k_{2}$ and $k_{1}, k_{2}=1,2,3$, the unit vector $\vec{e}_{k_{1}, k_{2}}$ is a constant vector with no numerical error during a time-marching procedure. As such with the aid of Eqs.(3.5), (3.21) and (3.40)-(3.43), and the definitions: (i)

$$
\nu_{x} \stackrel{\text { def }}{=} \frac{\partial \phi}{\partial x}=a \text { and } \nu_{y} \stackrel{\text { def }}{=} \frac{\partial \phi}{\partial y}=b \quad(\text { on } \Gamma)
$$

and (ii)

$$
\left.\overrightarrow{\Delta \nu} \stackrel{\text { def }}{=} \Delta \nu_{x} \vec{e}_{x}+\Delta \nu_{y} \vec{e}_{y} \quad \text { (on } \Gamma\right)
$$

where $\Delta \nu_{x}$ and $\Delta \nu_{y}$, respectively, denote the numerical errors of $\nu_{x}$ and $\nu_{y}$. One concludes from Eq.(3.29) that

$$
\overrightarrow{\Delta \nu} \bullet \vec{e}_{k}=\epsilon_{k}, k=1,2,3 \quad(\text { on } \Gamma)
$$

Also, because of the assumption Eq.(3.1), (i) Eqs.(3.26) and (3.41) $\Rightarrow$

$$
\vec{e}_{k_{1}} \neq \pm \vec{e}_{k_{2}} \text { if } k_{1} \neq k_{2} \text { and } k_{1}, k_{2}=1,2,3
$$

and (ii) the internal angles $\alpha_{1}, \alpha_{2}$ and $\alpha_{3}$ of $\Delta P_{1} P_{2} P_{3}$ depicted in Fig.3(a) must satisfy the condition (Note: the symbol " $\in$ " $\Leftrightarrow$ "belongs to" ):

$$
\left(\alpha_{1}, \alpha_{2}, \alpha_{3}\right) \in D_{\alpha}
$$

where 


$$
D_{\alpha} \stackrel{\text { def }}{=}\left\{\left(\alpha_{1}, \alpha_{2}, \alpha_{3}\right) \mid 0<\alpha_{1}, \alpha_{2}, \alpha_{3}<\pi \text { and } \alpha_{1}+\alpha_{2}+\alpha_{3}=\pi\right\}
$$

Moreover, with the aid of Eq.(3.46), we have

$$
|\overrightarrow{\Delta \nu}| \stackrel{\text { def }}{=} \sqrt{\overrightarrow{\Delta \nu} \bullet \overrightarrow{\Delta \nu}}=\sqrt{\left(\Delta \nu_{x}\right)^{2}+\left(\Delta \nu_{y}\right)^{2}}
$$

and thus

$$
(|\overrightarrow{\Delta \nu}|)^{2}=\left(\Delta \nu_{x}\right)^{2}+\left(\Delta \nu_{y}\right)^{2}
$$

With the above preliminary, we will prove the following theorem:

Theorem 1: Let (i) $\alpha_{1}, \alpha_{2}$, and $\alpha_{3}$ be the internal angles of $\Delta P_{1} P_{2} P_{3}$, shown in Fig.3(a) and thus $\left(\alpha_{1}, \alpha_{2}, \alpha_{3}\right) \in D_{\alpha}$, (ii)

$$
S(\alpha) \stackrel{\text { def }}{=} \frac{1}{\sin ^{2} \alpha}\left(\begin{array}{cc}
1 & \cos \alpha \\
\cos \alpha & 1
\end{array}\right), \quad 0<\alpha<\pi
$$

and (iii)

$$
\hat{\epsilon}_{1} \stackrel{\text { def }}{=}\left(\begin{array}{c}
\epsilon_{2} \\
\epsilon_{3}
\end{array}\right), \hat{\epsilon}_{2} \stackrel{\text { def }}{=}\left(\begin{array}{l}
\epsilon_{3} \\
\epsilon_{1}
\end{array}\right) \text { and } \hat{\epsilon}_{3} \stackrel{\text { def }}{=}\left(\begin{array}{c}
\epsilon_{1} \\
\epsilon_{2}
\end{array}\right)
$$

Then

$$
(|\overrightarrow{\Delta \nu}|)^{2}=\left(\hat{\epsilon}_{k}\right)^{t} S\left(\alpha_{k}\right) \hat{\epsilon}_{k}, \text { for each } k=1,2,3
$$

where for each $k=1,2,3,\left(\hat{\epsilon}_{k}\right)^{t}$ is the transpose of $\hat{\epsilon}_{k}$.

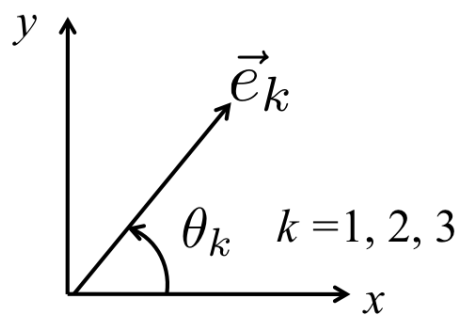

Figure 5. Representation of a unit vector in $x-y$ space.

Proof: First note that, for each $k=1,2,3$, (i) $\vec{e}_{k}$ is the unit vector defined by Eqs.(3.26) and (3.41), and (ii) the angle $\theta_{k}$, shown in Fig.5, is uniquely defined by $\vec{e}_{k}$ through the conditions:

$$
\vec{e}_{k}=\left(\cos \theta_{k}\right) \vec{e}_{x}+\left(\sin \theta_{k}\right) \vec{e}_{y}, \quad k=1,2,3
$$

and

$$
\pi \geq \theta_{k}>-\pi, \quad k=1,2,3
$$

Also it follows from Eq.(3.56) that

$$
\vec{e}_{k_{1}} \bullet \vec{e}_{k_{2}}=\left(\cos \theta_{k_{1}}\right)\left(\cos \theta_{k_{2}}\right)+\left(\sin \theta_{k_{1}}\right)\left(\sin \theta_{k_{2}}\right) \equiv \cos \left(\theta_{k_{2}}-\theta_{k_{1}}\right), k_{1}, k_{2}=1,2,3
$$

Because $\vec{e}_{k}, k=1,2,3$, are unit vectors, Eqs.(3.48) and (3.58) $\Rightarrow$

$$
\left(\vec{e}_{k_{1}} \bullet \vec{e}_{k_{2}}\right)=\left|\cos \left(\theta_{k_{2}}-\theta_{k_{1}}\right)\right|<1 \text { if } k_{1} \neq k_{2} \text { and } k_{1}, k_{2}=1,2,3
$$

which, in turn, $\Rightarrow$

$$
\sin \left(\theta_{k_{2}}-\theta_{k_{1}}\right) \neq 0 \text { if } k_{1} \neq k_{2} \text { and } k_{1}, k_{2}=1,2,3
$$


Note that, with the aid of Eq.(3.59), it can be shown that Eq.(3.48) $\Leftrightarrow$ Eq.(3.60). Moreover, because Eq.(3.57) $\Rightarrow$

$$
2 \pi>\theta_{k_{2}}-\theta_{k_{1}}>-2 \pi, \text { if } k_{1} \neq k_{2} \text { and } k_{1}, k_{2}=1,2,3
$$

As such, assuming Eq.(3.57), Eq.(3.48) $\Leftrightarrow$ Eq.(3.60) $\Leftrightarrow$

$$
\theta_{k_{2}}-\theta_{k_{1}} \neq \pm \pi \text { and } \theta_{k_{2}}-\theta_{k_{1}} \neq 0 \text { if } k_{1} \neq k_{2} \text { and } k_{1}, k_{2}=1,2,3
$$

Substituting Eqs.(3.46) and Eq.(3.56) into Eq.(3.47), one has

$$
\left(\cos \theta_{k}\right) \Delta \nu_{x}+\left(\sin \theta_{k}\right) \Delta \nu_{y}=\epsilon_{k} \text { for any } k=1,2,3
$$

On the other hand, given any pair of $k_{1}$ and $k_{2}$ with $k_{1} \neq k_{2}$ and $k_{1}, k_{2}=1,2,3$, in turn Eq.(3.63) $\Rightarrow$

$$
\left.\begin{array}{l}
\left(\cos \theta_{k_{1}}\right) \Delta \nu_{x}+\left(\sin \theta_{k_{1}}\right) \Delta \nu_{y}=\epsilon_{k_{1}} \\
\left(\cos \theta_{k_{2}}\right) \Delta \nu_{x}+\left(\sin \theta_{k_{2}}\right) \Delta \nu_{y}=\epsilon_{k_{2}}
\end{array}\right\}, k_{1} \neq k_{2} \text { and } k_{1}, k_{2}=1,2,3
$$

Because, with the aid of Eq.(3.60) one has

$$
\begin{array}{r}
\left|\begin{array}{rr}
\cos \theta_{k_{1}} & \sin \theta_{k_{1}} \\
\cos \theta_{k_{2}} & \sin \theta_{k_{2}}
\end{array}\right|=\left(\sin \theta_{k_{2}}\right)\left(\cos \theta_{k_{1}}\right)-\left(\cos \theta_{k_{2}}\right)\left(\sin \theta_{k_{1}}\right)=\sin \left(\theta_{k_{2}}-\theta_{k_{1}}\right) \neq 0 \\
\text { if } k_{1} \neq k_{2} \text { and } k_{1}, k_{2}=1,2,3
\end{array}
$$

Eq.(3.64) can be inverted to yield the following result

$$
\hat{\delta}=M\left(k_{1}, k_{2}\right) \hat{\epsilon}\left(k_{1}, k_{2}\right) \quad \text { if } k_{1} \neq k_{2} \text { and } k_{1}, k_{2}=1,2,3
$$

where (i)

$$
\hat{\delta} \stackrel{\text { def }}{=}\left(\begin{array}{c}
\Delta \nu_{x} \\
\Delta \nu_{y}
\end{array}\right)
$$

(ii)

$$
M\left(k_{1}, k_{2}\right) \stackrel{\text { def }}{=} \frac{1}{\sin \left(\theta_{k_{2}}-\theta_{k_{1}}\right)}\left(\begin{array}{cc}
\sin \theta_{k_{2}} & -\sin \theta_{k_{1}} \\
-\cos \theta_{k_{2}} & \cos \theta_{k_{1}}
\end{array}\right), k_{1} \neq k_{2} \text { and } k_{1}, k_{2}=1,2,3
$$

and (iii)

$$
\hat{\epsilon}\left(k_{1}, k_{2}\right) \stackrel{\text { def }}{=}\left(\begin{array}{c}
\epsilon_{k_{1}} \\
\epsilon_{k_{2}}
\end{array}\right), k_{1} \neq k_{2} \text { and } k_{1}, k_{2}=1,2,3
$$

Note that: (i) Eqs.(3.54) and (3.69) $\Rightarrow$

$$
\hat{\epsilon}_{1}=\hat{\epsilon}(2,3), \hat{\epsilon}_{2}=\hat{\epsilon}(3,1) \text { and } \hat{\epsilon}_{3}=\hat{\epsilon}(1,2)
$$

(ii) let $\left[M\left(k_{1}, k_{2}\right)\right]^{t}$ be the transpose of $M\left(k_{1}, k_{2}\right)$, then Eq.(3.68) $\Rightarrow$

$$
\begin{array}{r}
T\left(k_{1}, k_{2}\right) \stackrel{\text { def }}{=}\left[M\left(k_{1}, k_{2}\right)\right]^{t} M\left(k_{1}, k_{2}\right)=\frac{1}{\sin ^{2}\left(\theta_{k_{2}}-\theta_{k_{1}}\right)}\left(\begin{array}{cc}
1 & -\cos \left(\theta_{k_{2}}-\theta_{k_{1}}\right) \\
-\cos \left(\theta_{k_{2}}-\theta_{k_{1}}\right) & 1
\end{array}\right) \\
\text { for any }\left(k_{1}, k_{2}\right) \text { with } k_{1} \neq k_{2} \text { and } k_{1}, k_{2}=1,2,3
\end{array}
$$

and (iii) Eqs.(3.52), (3.66), (3.67) and (3.71) $\Rightarrow$

$$
\begin{array}{r}
(|\overrightarrow{\Delta \nu}|)^{2}=\left(\Delta \nu_{x}\right)^{2}+ \\
\left.\left.\left.=\left[\Delta \nu_{y}\right)^{2}=(\hat{\delta})^{t} \hat{\delta}=\left[\hat{\epsilon}\left(k_{1}, k_{2}\right)\right]^{t}\left[M\left(k_{1}\right)\right]^{t} T\left(k_{2}\right)\right]^{t} M\left(k_{2}\right)\right] \hat{\epsilon}\left(k_{1}, k_{2}\right), k_{2}\right) \hat{\epsilon}\left(k_{1}, k_{2}\right) \\
=k_{2} \text { and } k_{1}, k_{2}=1,2,3
\end{array}
$$

where, $\hat{\delta}^{t}$ denotes the transpose of $\hat{\delta}$. 
Note that: (i) Eq.(3.69) $\Rightarrow$

$$
\hat{\epsilon}\left(k_{2}, k_{1}\right)=P^{\wedge} \epsilon\left(k_{1}, k_{2}\right) \text {, for any }\left(k_{1}, k_{2}\right) \text { with } k_{1} \neq k_{2} \text { and } k_{1}, k_{2}=1,2,3
$$

where the $2 \times 2$ permutation matrix

$$
P \stackrel{\text { def }}{=}\left(\begin{array}{ll}
0 & 1 \\
1 & 0
\end{array}\right)
$$

has the property

$$
P^{-1}=P^{t}=P
$$

where $P^{-1}$ is the inverse of $P$; (ii) Eq.(3.71) $\Rightarrow$

$$
T\left(k_{2}, k_{1}\right)=T\left(k_{1}, k_{2}\right), \quad k_{1} \neq k_{2} \text { and } k_{1}, k_{2}=1,2,3
$$

(iii) with the aid of Eqs.(3.74) and (3.75), Eq.(3.71) $\Rightarrow$

$$
P^{t} T\left(k_{1}, k_{2}\right) P=T\left(k_{1}, k_{2}\right), \quad k_{1} \neq k_{2} \text { and } k_{1}, k_{2}=1,2,3
$$

and (iv) Eqs.(3.73), (3.76), and (3.77) $\Rightarrow$

$$
\begin{array}{r}
\left.\left[\hat{\epsilon}\left(k_{2}, k_{1}\right)\right]^{t} T\left(k_{2}, k_{1}\right)\right] \hat{\epsilon}\left(k_{2}, k_{1}\right)=\left[\hat{\epsilon}\left(k_{1}, k_{2}\right)\right]^{t} T\left(k_{1}, k_{2}\right) \hat{\epsilon}\left(k_{1}, k_{2}\right) \\
k_{1} \neq k_{2} \text { and } k_{1}, k_{2}=1,2,3
\end{array}
$$

Because of the relation Eq. (3.78), Eq.(3.72) represents only three independent relations. As such, with the aid of Eq.(3.70), one concludes that Eq.(3.72) $\Leftrightarrow$

$$
\left(|\overrightarrow{\Delta \nu}|^{2}\right)=\left(\hat{\epsilon}_{1}\right)^{t} T(2,3) \hat{\epsilon}_{1}=\left(\hat{\epsilon}_{2}\right)^{t} T(3,1) \hat{\epsilon}_{2}=\left(\hat{\epsilon}_{3}\right)^{t} T(1,2) \hat{\epsilon}_{3}
$$

By comparing Eq.(3.79) with Eq.(3.55) it is seen that the proof of Theorem 1 is completed if one can show that

$$
T(2,3)=S\left(\alpha_{1}\right), T(3,1)=S\left(\alpha_{2}\right) \text { and } T(1,2)=S\left(\alpha_{3}\right)
$$

To prove Eq.(3.80), note that Eqs.(3.25), (3.26), and (3.41) $\Rightarrow$

$$
\overrightarrow{e_{1}}=\frac{\overrightarrow{P_{2} P_{3}}}{\left|\overrightarrow{P_{2} P_{3}}\right|}, \overrightarrow{e_{2}}=\frac{\overrightarrow{P_{3} P_{1}}}{\left|\overrightarrow{P_{3} P_{1}}\right|} \text { and } \overrightarrow{e_{3}}=\frac{\overrightarrow{P_{1} P_{2}}}{\left|\overrightarrow{P_{1} P_{2}}\right|}
$$

In turn, with the aid of Fig.3(a) and Eq.(3.81), one has

$$
-\vec{e}_{2} \bullet \vec{e}_{3}=\frac{\overrightarrow{P_{1} P_{3}} \bullet \overrightarrow{P_{1} P_{2}}}{\left|\overrightarrow{P_{1} P_{3}}\right|\left|\overrightarrow{P_{1} P_{2}}\right|}=\frac{\left|\overrightarrow{P_{1} P_{3}}\right|\left|\overrightarrow{P_{1} P_{2}}\right| \cos \alpha_{1}}{\left|\overrightarrow{P_{1} P_{3}}\right|\left|\overrightarrow{P_{1} P_{2}}\right|}=\cos \alpha_{1}
$$

On the other hand, by using Eqs.(3.58) and elementary trigonometry, one has

$$
-\vec{e}_{2} \bullet \vec{e}_{3}=-\cos \left(\theta_{3}-\theta_{2}\right)=-\cos \left(\theta_{2}-\theta_{3}\right)
$$

By using Eqs.(3.82) and (3.83) along with elementary trigonometry, one has

$$
-\cos \left(\theta_{2}-\theta_{3}\right)=\cos \alpha_{1} \text { and } \sin ^{2}\left(\theta_{2}-\theta_{3}\right)=\sin ^{2} \alpha_{1}
$$

Moreover, by using Eqs.(3.60), (3.71) and (3.76), one concludes that (i) $\sin \left(\theta_{2}-\theta_{3}\right) \neq 0$, and (ii)

$$
T(2,3)=T(3,2)=\frac{1}{\sin ^{2}\left(\theta_{2}-\theta_{3}\right)}\left(\begin{array}{cc}
1 & -\cos \left(\theta_{2}-\theta_{3}\right) \\
-\cos \left(\theta_{2}-\theta_{3}\right) & 1
\end{array}\right)
$$

Combining Eqs.(3.53), (3.84), and (3.85) one arrives at the first part of Eq.(3.80), i.e., $T(2,3)=S\left(\alpha_{1}\right)$. Moreover, because the new version of proof emerging from the above proof for $T(2,3)=S\left(\alpha_{1}\right)$ 
after it undergoes the index permutation of Eq.(3.44) remains valid, one will arrive at the second part of Eq.(3.80). Similarly one can prove the third part of Eq.(3.80). Because Eqs.(3.79) and (3.80) $\Rightarrow$ Eq.(3.55), the proof of Theorem 1 is completed. QED.

As a preliminary for the following developments, note that, by using (i) Fig.3(a), (ii) Eq.(3.25), and (iii) the law of the sines, one concludes that: (i)

$$
\sin \alpha_{k}>0, k=1,2,3, \text { for each }\left(\alpha_{1}, \alpha_{2}, \alpha_{3}\right) \in D_{\alpha}
$$

and (ii) there exists a parameter $\beta>0$ such that

$$
\frac{s_{2,3}}{\sin \alpha_{1}}=\frac{s_{3,1}}{\sin \alpha_{2}}=\frac{s_{1,2}}{\sin \alpha_{3}}=\beta>0 \text { for each }\left(\alpha_{1}, \alpha_{2}, \alpha_{3}\right) \in D_{\alpha}
$$

where $D_{\alpha}$ is defined in Eq.(3.50). Next, by using Eq.(3.40) and an equivalent of Eq.(3.87), i.e.,

$$
s_{2,3}=\beta \sin \alpha_{1}, s_{3,1}=\beta \sin \alpha_{2} \text { and } s_{1,2}=\beta \sin \alpha_{3} \quad\left(\beta>0 \text { and }\left(\alpha_{1}, \alpha_{2}, \alpha_{3}\right) \in D_{\alpha}\right)
$$

Eq.(3.38) can be recast as

$$
\left(\sin \alpha_{1}\right) \epsilon_{1}+\left(\sin \alpha_{2}\right) \epsilon_{2}+\left(\sin \alpha_{3}\right) \epsilon_{3}=0,\left(\alpha_{1}, \alpha_{2}, \alpha_{3}\right) \in D_{\alpha}
$$

Next, for a given $\left(\alpha_{1}, \alpha_{2}, \alpha_{3}\right) \in D_{\alpha}$, let

$$
\begin{array}{r}
\Lambda\left(\alpha_{1}, \alpha_{2}, \alpha_{3}\right)=\left\{\left(\epsilon_{1}, \epsilon_{2}, \epsilon_{3}\right) \mid \epsilon_{1}, \epsilon_{2} \text { and } \epsilon_{3}\right. \text { be real parameters } \\
\text { satisfying Eq.(3.89) }\} \quad\left(\alpha_{1}, \alpha_{2}, \alpha_{3}\right) \in D_{\alpha}
\end{array}
$$

i.e., for a given $\left(\alpha_{1}, \alpha_{2}, \alpha_{3}\right) \in D_{\alpha}, \Lambda\left(\alpha_{1}, \alpha_{2}, \alpha_{3}\right)$ contains all the elements $\left(\epsilon_{1}, \epsilon_{2}, \epsilon_{3}\right)$ which satisfy Eq.(3.89). In turn, with the aid of Eq.(3.86), one concludes that, for any $\left(\epsilon_{1}, \epsilon_{2}, \epsilon_{3}\right) \in \Lambda\left(\alpha_{1}, \alpha_{2}, \alpha_{3}\right)$ with $\left(\alpha_{1}, \alpha_{2}, \alpha_{3}\right) \in D_{\alpha}$, any one of $\epsilon_{1}, \epsilon_{2}$, and $\epsilon_{3}$, can be uniquely determined in terms of the other two by using Eq.(3.89). In fact, for any $\left(\hat{\epsilon}_{1}, \hat{\epsilon}_{2}, \hat{\epsilon}_{3}\right)$ defined from any given $\left(\epsilon_{1}, \epsilon_{2}, \epsilon_{3}\right) \in \Lambda\left(\alpha_{1}, \alpha_{2}, \alpha_{3}\right)$ using Eq.(3.54), Eq.(3.89) $\Leftrightarrow$ any one of the following six relations:

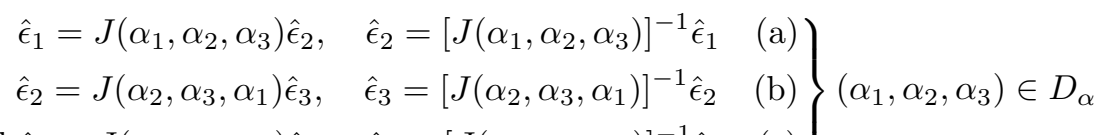

$$
\begin{aligned}
& \text { and } \left.\hat{\epsilon}_{3}=J\left(\alpha_{3}, \alpha_{1}, \alpha_{2}\right) \hat{\epsilon}_{1}, \quad \hat{\epsilon}_{1}=\left[J\left(\alpha_{3}, \alpha_{1}, \alpha_{2}\right)\right]^{-1} \hat{\epsilon}_{3} \quad(\mathrm{c})\right)
\end{aligned}
$$

Here, for any $\left(\alpha_{1}, \alpha_{2}, \alpha_{3}\right) \in D_{\alpha}\left[\right.$ which $\left.\Leftrightarrow\left(\alpha_{2}, \alpha_{3}, \alpha_{1}\right) \in D_{\alpha} \Leftrightarrow\left(\alpha_{3}, \alpha_{1}, \alpha_{2}\right) \in D_{\alpha}\right]$, (i)

$$
J\left(\alpha_{1}, \alpha_{2}, \alpha_{3}\right) \stackrel{\text { def }}{=} \frac{1}{\sin \alpha_{2}}\left(\begin{array}{cc}
-\sin \alpha_{3} & -\sin \alpha_{1} \\
\sin \alpha_{2} & 0
\end{array}\right),\left(\alpha_{1}, \alpha_{2}, \alpha_{3}\right) \in D_{\alpha}
$$

and (ii) $\left[J\left(\alpha_{1}, \alpha_{2}, \alpha_{3}\right)\right]^{-1}$, is explicitly given by

$$
\left[J\left(\alpha_{1}, \alpha_{2}, \alpha_{3}\right)\right]^{-1}=\frac{1}{\sin \alpha_{1}}\left(\begin{array}{cc}
0 & \sin \alpha_{1} \\
-\sin \alpha_{2} & -\sin \alpha_{3}
\end{array}\right),\left(\alpha_{1}, \alpha_{2}, \alpha_{3}\right) \in D_{\alpha}
$$

As a preliminary for a key future development, given any $\left(\alpha_{1}, \alpha_{2}, \alpha_{3}\right) \in D_{\alpha}$, next we will prove the following relations:

$$
\begin{aligned}
& S\left(\alpha_{1}\right)=\left[\left(J\left(\alpha_{1}, \alpha_{2}, \alpha_{3}\right)\right)^{-1}\right]^{t} S\left(\alpha_{2}\right)\left(J\left(\alpha_{1}, \alpha_{2}, \alpha_{3}\right)\right)^{-1} \\
& S\left(\alpha_{2}\right)=\left[\left(J\left(\alpha_{2}, \alpha_{3}, \alpha_{1}\right)\right)^{-1}\right]^{t} S\left(\alpha_{3}\right)\left(J\left(\alpha_{2}, \alpha_{3}, \alpha_{1}\right)\right)^{-1} \\
& \text { and } S\left(\alpha_{3}\right)=\left[\left(J\left(\alpha_{3}, \alpha_{1}, \alpha_{2}\right)\right)^{-1}\right]^{t} S\left(\alpha_{1}\right)\left(J\left(\alpha_{3}, \alpha_{1}, \alpha_{2}\right)\right)^{-1}
\end{aligned}
$$

Proof for Eq.(3.94): By using Eqs.(3.53) and (3.93), one has

$$
\begin{array}{r}
{\left[\left(J\left(\alpha_{1}, \alpha_{2}, \alpha_{3}\right)\right)^{-1}\right]^{t} S\left(\alpha_{2}\right)\left(J\left(\alpha_{1}, \alpha_{2}, \alpha_{3}\right)\right)^{-1}} \\
\left(\begin{array}{cc}
\sin ^{2} \alpha_{2} & \frac{1}{\sin ^{2} \alpha_{1} \cdot \sin ^{2} \alpha_{2}} \times \\
\sin \alpha_{2}\left(\sin \alpha_{3}-\sin \alpha_{1} \cos \alpha_{2}\right) \\
\sin \alpha_{2}\left(\sin \alpha_{3}-\sin \alpha_{1} \cos \alpha_{2}\right) & \sin ^{2} \alpha_{1}+\sin \alpha_{3}\left(\sin \alpha_{3}-2 \sin \alpha_{1} \cos \alpha_{2}\right)
\end{array}\right),\left(\alpha_{1}, \alpha_{2}, \alpha_{3}\right) \in D_{\alpha}
\end{array}
$$


To simplify Eq.(3.95) note that Eqs.(3.49) and (3.50) $\Rightarrow \alpha_{3}=\pi-\alpha_{1}-\alpha_{2}$ and thus

$$
\sin \alpha_{3}=\sin \left(\pi-\alpha_{1}-\alpha_{2}\right)=\sin \left(\alpha_{1}+\alpha_{2}\right)=\sin \alpha_{1} \cos \alpha_{2}+\sin \alpha_{2} \cos \alpha_{1}
$$

In turn, by using Eq.(3.96) and some trignometric identities, one has

$$
\sin \alpha_{2}\left(\sin \alpha_{3}-\sin \alpha_{1} \cos \alpha_{2}\right)=\left(\sin ^{2} \alpha_{2}\right) \cos \alpha_{1}
$$

and

$$
\sin ^{2} \alpha_{1}+\sin \alpha_{3}\left(\sin \alpha_{3}-2 \sin \alpha_{1} \cos \alpha_{2}\right)=\sin ^{2} \alpha_{2}
$$

Next, by substituting Eqs.(3.97) and (3.98) into Eq.(3.95) and then using Eq.(3.53) again, one arrives at Eq.(3.94)(a). Because Eq.(3.94)(b) is the image of Eq.(3.94)(a) under the index permutation of Eq.(3.44), by carrying out this permutation over the proof for Eq.(3.94)(a), one has the proof for Eq.(3.94)(b). Similarly one can prove Eq.(3.94)(c). QED.

Note that an immediate result of Eq.(3.55) is

$$
\left(\hat{\epsilon}_{1}\right)^{t} S\left(\alpha_{1}\right) \hat{\epsilon}_{1}=\left(\hat{\epsilon}_{2}\right)^{t} S\left(\alpha_{2}\right) \hat{\epsilon}_{2}=\left(\hat{\epsilon}_{3}\right)^{t} S\left(\alpha_{3}\right) \hat{\epsilon}_{3},\left(\alpha_{1}, \alpha_{2}, \alpha_{3}\right) \in D_{\alpha}
$$

In the following, Eq.(3.99) is proved directly using Eqs.(3.91) and (3.94).

Proof for Eq.(3.99): By using the second part of Eq.(3.91)(a), one has

$$
\left(\hat{\epsilon}_{2}\right)^{t} S\left(\alpha_{2}\right) \hat{\epsilon}_{2}=\left(\hat{\epsilon}_{1}\right)^{t}\left[\left(J\left(\alpha_{1}, \alpha_{2}, \alpha_{3}\right)\right)^{-1}\right]^{t} S\left(\alpha_{2}\right)\left(J\left(\alpha_{1}, \alpha_{2}, \alpha_{3}\right)\right)^{-1} \hat{\epsilon}_{1}
$$

In turn, by substituting Eq.(3.94)(a) into Eq.(3.100), one has

$$
\left(\hat{\epsilon}_{2}\right)^{t} S\left(\alpha_{2}\right) \hat{\epsilon}_{2}=\left(\hat{\epsilon}_{1}\right)^{t} S\left(\alpha_{1}\right) \hat{\epsilon}_{1}
$$

i.e., the validity of the first equality sign in Eq.(3.99) has been proved. Similary, one can prove the validity of the second equality sign. QED.

Moreover, Eq.(3.91) implies that any $\left(\hat{\epsilon}_{1}, \hat{\epsilon}_{2}, \hat{\epsilon}_{3}\right)$ which are defined in terms of the same $\left(\epsilon_{1}, \epsilon_{2}, \epsilon_{3}\right) \in$ $\Lambda\left(\alpha_{1}, \alpha_{2}, \alpha_{3}\right)$ using Eq.(3.54) has the property that $\hat{\epsilon}_{k}=0$ for any $k=1,2,3 \Leftrightarrow \hat{\epsilon}_{1}=\hat{\epsilon}_{2}=\hat{\epsilon}_{3}=0$. As such, any $\left(\hat{\epsilon}_{1}, \hat{\epsilon}_{2}, \hat{\epsilon}_{3}\right)$ defined using the same $\left(\epsilon_{1}, \epsilon_{2}, \epsilon_{3}\right) \in \Lambda\left(\alpha_{1}, \alpha_{2}, \alpha_{3}\right)$ must meet one of the following two mutually exclusive conditions:

$$
\text { (a) } \hat{\epsilon}_{k}=0 \text {, for each } k=1,2,3
$$

and

$$
\text { (b) } \hat{\epsilon}_{k} \neq 0 \text {, i.e., }\left(\hat{\epsilon}_{k}\right)^{t} \hat{\epsilon}_{k}>0 \text {, for each } k=1,2,3
$$

With the aid of Eq.(3.54), it is seen that Eq.(3.102) $\Leftrightarrow$

$$
\epsilon_{1}=\epsilon_{2}=\epsilon_{3}=0
$$

According to Eqs.(3.35)-(3.37) and (3.40), Eq.(3.104) implies that the numerical errors of the directional derivatives of $\phi$ evaluated along the three sides of $\Delta P_{1} P_{2} P_{3}$ are all zero.

Because (i) Eq.(3.102) $\Leftrightarrow$ Eq.(3.104), (ii) Eq.(3.104) and the condition

$$
\epsilon^{*} \stackrel{\text { def }}{=} \sqrt{\left(\epsilon_{1}\right)^{2}+\left(\epsilon_{2}\right)^{2}+\left(\epsilon_{3}\right)^{2}}>0
$$

are mutually exclusive, and (iii) any $\left(\hat{\epsilon}_{1}, \hat{\epsilon}_{2}, \hat{\epsilon}_{3}\right)$ defined in terms of the same $\left(\epsilon_{1}, \epsilon_{2}, \epsilon_{3}\right) \in \Lambda\left(\alpha_{1}, \alpha_{2}, \alpha_{3}\right)$ must belong to one of the two exclusive cases, Eqs.(3.102) and (3.103), one concludes that Eq.(3.103) $\Leftrightarrow$ Eq.(3.105).

Because the case Eq.(3.104) is numerically unrealistic and, according to Eqs.(3.54) and (3.55), it leads to the trivial result $\Delta \nu_{x}=\Delta \nu_{y}=0$ (which, according to Eqs.(3.40)-(3.43) and (3.45)-(3.47), simply states that the numerical error of the vector $\vec{\nabla} \phi$ on the plane $\Gamma$ is zero if the numerical errors $\epsilon_{1}, \epsilon_{2}$ and $\epsilon_{3}$ of the directional derivatives of $\phi$ evaluated along the three sides of $\Delta P_{1} P_{2} P_{3}$ are all zero), hereafter, for any given $\left(\alpha_{1}, \alpha_{2}, \alpha_{3}\right) \in D_{\alpha}$, unless specified otherwise, we will consider only the case Eq.(3.103), i.e., only the case

$$
\left(\epsilon_{1}, \epsilon_{2}, \epsilon_{3}\right) \in \Lambda^{\prime}\left(\alpha_{1}, \alpha_{2}, \alpha_{3}\right), \quad\left(\alpha_{1}, \alpha_{2}, \alpha_{3}\right) \in D_{\alpha}
$$


where,

$$
\Lambda^{\prime}\left(\alpha_{1}, \alpha_{2}, \alpha_{3}\right) \stackrel{\text { def }}{=}\left\{\left(\epsilon_{1}, \epsilon_{2}, \epsilon_{3}\right) \mid\left(\epsilon_{1}, \epsilon_{2}, \epsilon_{3}\right) \in \Lambda\left(\alpha_{1}, \alpha_{2}, \alpha_{3}\right)\right. \text { and also satisfies }
$$

the condition Eq.(3.103) or its equivalent $\left.\epsilon^{*}>0\right\}$ for any $\left(\alpha_{1}, \alpha_{2}, \alpha_{3}\right) \in D_{\alpha}$

Next, with the aid of Eqs.(3.86), (3.89) and (3.105), we will prove the following theorem:

Theorem 2: Let (a) $\left(\alpha_{1}, \alpha_{2}, \alpha_{3}\right) \in D_{\alpha}$, and thus Eq.(3.86) $\Rightarrow$

$$
C_{k, l} \stackrel{\text { def }}{=} \frac{\sin \alpha_{l}}{\sin \alpha_{k}}>0, k \neq l \text { and } k, l=1,2,3
$$

(b)

$$
\begin{aligned}
& E_{1} \stackrel{\text { def }}{=}\left(\begin{array}{cc}
1+\left(C_{1,2}\right)^{2} & C_{1,2} \cdot C_{1,3} \\
C_{1,2} \cdot C_{1,3} & 1+\left(C_{1,3}\right)^{2}
\end{array}\right) \\
& E_{2} \stackrel{\text { def }}{=}\left(\begin{array}{cc}
1+\left(C_{2,3}\right)^{2} & C_{2,3} \cdot C_{2,1} \\
C_{2,3} \cdot C_{2,1} & 1+\left(C_{2,1}\right)^{2}
\end{array}\right)
\end{aligned}
$$

and

$$
E_{3} \stackrel{\text { def }}{=}\left(\begin{array}{cc}
1+\left(C_{3,1}\right)^{2} & C_{3,1} \cdot C_{3,2} \\
C_{3,1} \cdot C_{3,2} & 1+\left(C_{3,2}\right)^{2}
\end{array}\right)
$$

(c)

$$
\begin{aligned}
& \lambda_{1+} \stackrel{\text { def }}{=} 1+\left(C_{1,2}\right)^{2}+\left(C_{1,3}\right)^{2}, \lambda_{1-} \stackrel{\text { def }}{=} 1 \\
& \lambda_{2+} \stackrel{\text { def }}{=} 1+\left(C_{2,3}\right)^{2}+\left(C_{2,1}\right)^{2}, \lambda_{2-} \stackrel{\text { def }}{=} 1
\end{aligned}
$$

and

$$
\lambda_{3+} \stackrel{\text { def }}{=} 1+\left(C_{3,1}\right)^{2}+\left(C_{3,2}\right)^{2}, \lambda_{3-} \stackrel{\text { def }}{=} 1
$$

(d)

$$
\begin{aligned}
& W_{1} \stackrel{\text { def }}{=} \frac{1}{\sqrt{\left(C_{1,2}\right)^{2}+\left(C_{1,3}\right)^{2}}}\left(\begin{array}{cc}
\sqrt{\lambda_{1+}} C_{1,2} & \sqrt{\lambda_{1+}} C_{1,3} \\
C_{1,3} & -C_{1,2}
\end{array}\right) \\
& W_{2} \stackrel{\text { def }}{=} \frac{1}{\sqrt{\left(C_{2,3}\right)^{2}+\left(C_{2,1}\right)^{2}}}\left(\begin{array}{cc}
\sqrt{\lambda_{2+}} C_{2,3} & \sqrt{\lambda_{2+}} C_{2,1} \\
C_{2,1} & -C_{2,3}
\end{array}\right)
\end{aligned}
$$

and

$$
W_{3} \stackrel{\text { def }}{=} \frac{1}{\sqrt{\left(C_{3,1}\right)^{2}+\left(C_{3,2}\right)^{2}}}\left(\begin{array}{cc}
\sqrt{\lambda_{3+}} C_{3,1} & \sqrt{\lambda_{3+}} C_{3,2} \\
C_{3,2} & -C_{3,1}
\end{array}\right)
$$

and (e)

$$
\hat{\psi}_{k} \stackrel{\text { def }}{=} W_{k} \hat{\epsilon}_{k}, k=1,2,3
$$

where $\hat{\epsilon}_{k}, k=1,2,3$, are defined by Eq.(3.54) and by assumption, belong to the case Eq.(3.103). Then we have:

(a) The real symmetric matrices $E_{1}, E_{2}$ and $E_{3}$ are all positive definite [Ref.9, p.250].

$$
\left(\epsilon^{*}\right)^{2}=\left(\hat{\epsilon}_{k}\right)^{t} E_{k} \hat{\epsilon}_{k}>0 \text { for each } k=1,2,3 \text { and each }\left(\epsilon_{1}, \epsilon_{2}, \epsilon_{3}\right) \in \Lambda^{\prime}\left(\alpha_{1}, \alpha_{2}, \alpha_{3}\right)
$$

(c)

$$
E_{k}=\left(W_{k}\right)^{t} W_{k}, k=1,2,3
$$

and $(\mathrm{d})$

$$
\left(\epsilon^{*}\right)^{2}=\left(\hat{\psi}_{k}\right)^{t} \hat{\psi}_{k}>0 \text { for each } k=1,2,3, \text { and each }\left(\epsilon_{1}, \epsilon_{2}, \epsilon_{3}\right) \in \Lambda^{\prime}\left(\alpha_{1}, \alpha_{2}, \alpha_{3}\right)
$$

Proof: Note that, for each $k=1,2,3, \lambda_{k+}$ and $\lambda_{k-}$ defined in one of Eqs.(3.112)-(3.114) are the eigenvalues of the real symmetric matrix $E_{k}$, defined in one of Eqs.(3.109)-(3.111). On the other hand, according to Eqs.(3.108) and (3.112)-(3.114), we have

$$
\lambda_{k+}>\lambda_{k-}=1, \text { for each } k=1,2,3
$$


Thus, for each $k=1,2,3$, the eigenvalues of $E_{k}$ are positive. As a result, by definition [Ref.9, p.250], for each $k=1,2,3$, the real symmetric matrix $E_{k}$ is positive definite. In turn, this coupled with Eq.(3.103) implies that [Ref.9, p.250]:

$$
\left(\hat{\epsilon}_{k}\right)^{t} E_{k} \hat{\epsilon}_{k}>0 \text { for each } k=1,2,3, \text { and each }\left(\epsilon_{1}, \epsilon_{2}, \epsilon_{3}\right) \in \Lambda^{\prime}\left(\alpha_{1}, \alpha_{2}, \alpha_{3}\right)
$$

Next, note that Eqs.(3.89) and (3.108) $\Rightarrow$

$$
\epsilon_{1}=-\left(C_{1,2} \epsilon_{2}+C_{1,3} \epsilon_{3}\right), \text { with } C_{1,2}>0 \text { and } C_{1,3}>0
$$

Then, by using Eqs.(3.54), (3.105), (3.109), (3.123) and (3.124), one concludes that

$$
\begin{array}{r}
\left(\epsilon^{*}\right)^{2}=\left[1+\left(C_{1,2}\right)^{2}\right]\left(\epsilon_{2}\right)^{2}+\left[1+\left(C_{1,3}\right)^{2}\right]\left(\epsilon_{3}\right)^{2}+2 C_{1,2} \cdot C_{1,3} \cdot\left(\epsilon_{2} \epsilon_{3}\right) \\
\equiv\left(\hat{\epsilon}_{1}\right)^{t} E_{1} \hat{\epsilon}_{1}>0 \text { for each }\left(\epsilon_{1}, \epsilon_{2}, \epsilon_{3}\right) \in \Lambda^{\prime}\left(\alpha_{1}, \alpha_{2}, \alpha_{3}\right)
\end{array}
$$

i.e., the $k=1$ case of Eq.(3.119) has been proved. To prove the $k=1$ case of Eqs.(3.120) and (3.121), note that (i)

$$
\hat{\epsilon}_{1+} \stackrel{\text { def }}{=} \frac{1}{\sqrt{\left(C_{1,2}\right)^{2}+\left(C_{1,3}\right)^{2}}}\left(\begin{array}{c}
C_{1,2} \\
C_{1,3}
\end{array}\right) \text { and } \hat{\epsilon}_{1-} \stackrel{\text { def }}{=} \frac{1}{\sqrt{\left(C_{1,2}\right)^{2}+\left(C_{1,3}\right)^{2}}}\left(\begin{array}{c}
C_{1,3} \\
-C_{1,2}
\end{array}\right)
$$

are the eigenvectors of $E_{1}$ with eigenvalues $\lambda_{1+}$ and $\lambda_{1-}$ respectively; and (ii)

$$
\left(\hat{\epsilon}_{1+}\right)^{t} \hat{\epsilon}_{1+}=\left(\hat{\epsilon}_{1-}\right)^{t} \hat{\epsilon}_{1-}=1
$$

Moreover, as expected from Eq.(3.122), and a matrix theorem, i.e., two eigenvectors of a real symmetric matrix with different eigenvalues must be orthogonal to each other [Ref.9, p.222], we have

$$
\left(\hat{\epsilon}_{1+}\right)^{t} \hat{\epsilon}_{1-}=\left(\hat{\epsilon}_{1-}\right)^{t} \hat{\epsilon}_{1+}=0
$$

Because $\hat{\epsilon}_{1+}$ and $\hat{\epsilon}_{1-}$ satisfy Eqs. (3.127) and (3.128), by definition [Ref.9, p.122], they form a pair of orthonormal eigenvectors of the matrix $E_{1}$.

Let,

$$
U_{1} \stackrel{\text { def }}{=} \frac{1}{\sqrt{\left(C_{1,2}\right)^{2}+\left(C_{1,3}\right)^{2}}}\left(\begin{array}{cc}
C_{1,2} & C_{1,3} \\
C_{1,3} & -C_{1,2}
\end{array}\right)
$$

i.e., the eigenvector $\hat{\epsilon}_{1+}$ and $\hat{\epsilon}_{1-}$ of $E_{1}$ respectively, are the first and second column of matrix $U_{1}$. By using orthorormal property of $\hat{\epsilon}_{1+}$ and $\hat{\epsilon}_{1-}$, it can be shown that

$$
\left(U_{1}\right)^{t} U_{1}=U_{1}\left(U_{1}\right)^{t}=\left(U_{1}\right)^{2}=I_{2} \stackrel{\text { def }}{=} \text { the } 2 \times 2 \text { identity matrix }
$$

Thus $U_{1}$ is a real symmetric orthogonal matrix [Ref.9, p.126] satisfying the relation

$$
\left(U_{1}\right)^{-1}=\left(U_{1}\right)^{t}=U_{1}
$$

Moreover, because $U_{1}$ is formed using two orthonormal eigenvectors $\hat{\epsilon}_{1+}$ and $\hat{\epsilon}_{1-}$ of $E_{1}$, the latter can be diagonalized through a similarity transformation involving $U_{1}$ [Ref.9, p.223], i.e.,

$$
\left(U_{1}\right)^{-1} E_{1} U_{1}=\left(\begin{array}{cc}
\lambda_{1+} & 0 \\
0 & \lambda_{1-}
\end{array}\right)=\left(\begin{array}{cc}
\lambda_{1+} & 0 \\
0 & 1
\end{array}\right)
$$

where $\lambda_{1+}$ and $\lambda_{1-}$ are the eigenvalues of $E_{1}$ defined in Eq.(3.112). Moreover, because $\lambda_{1+}>\lambda_{1-}=1$ and thus $\sqrt{\lambda_{1+}}>1$, Eqs.(3.131) and (3.132) $\Rightarrow$

$$
E_{1}=U_{1}\left(\begin{array}{cc}
\lambda_{1+} & 0 \\
0 & 1
\end{array}\right)\left(U_{1}\right)^{-1}=\left(U_{1}\right)^{t}\left(\begin{array}{cc}
\sqrt{\lambda_{1+}} & 0 \\
0 & 1
\end{array}\right)^{2} U_{1}
$$

Moreover, because Eqs.(3.115) and (3.129) $\Rightarrow$

$$
W_{1}=\left(\begin{array}{cc}
\sqrt{\lambda_{1+}} & 0 \\
0 & 1
\end{array}\right) U_{1}
$$

\section{8 of 38}


Eq.(3.133) now implies the $k=1$ case of Eq.(3.120), i.e.,

$$
E_{1}=\left(U_{1}\right)^{t}\left(\begin{array}{cc}
\sqrt{\lambda_{1+}} & 0 \\
0 & 1
\end{array}\right)\left(\begin{array}{cc}
\sqrt{\lambda_{1+}} & 0 \\
0 & 1
\end{array}\right) U_{1}=\left(W_{1}\right)^{t} W_{1}
$$

Next, by combining Eqs. (3.125), (3.135) and (3.118), one has

$$
\left(\epsilon^{*}\right)^{2}=\left(\hat{\epsilon}_{1}\right)^{t} E_{1} \hat{\epsilon}_{1}=\left(\hat{\epsilon}_{1}\right)^{t}\left(W_{1}\right)^{t} W_{1} \hat{\epsilon}_{1}=\left(W_{1} \hat{\epsilon}_{1}\right)^{t} W_{1} \hat{\epsilon}_{1}=\left(\hat{\psi}_{1}\right)^{t} \hat{\psi}_{1}>0 \text { for the case Eq. (3.103) }
$$

i.e, the $k=1$ case of Eq.(3.121) has been proved.

To prove Eqs.(3.119)-(3.121) for the $k=2$ case, note that new version of proof emerging from the above proof for the $k=1$ case after its component-identifications indices 1, 2, and 3 undergo the index permutation of Eq.(3.44) remain valid. In particular, after this permutation, Eqs.(3.125), (3.135) and (3.136), respectively, become

$$
\left(\epsilon^{*}\right)^{2}=\left(\hat{\epsilon}_{2}\right)^{t} E_{2} \hat{\epsilon}_{2}>0, E_{2}=\left(W_{2}\right)^{t}\left(W_{2}\right) \text { and }\left(\epsilon^{*}\right)^{2}=\left(\hat{\psi}_{2}\right)^{t} \hat{\psi}_{2}>0
$$

i.e., the $k=2$, case of Eqs.(3.119)-(3.121) has been proved. Similarly, the $k=3$ case can be proved by carrying out the index permutation of Eq.(3.44) over the proof for $k=2$ case. As such, Theorem 2 has been proved. QED.

Next, the following corollary follows directly from Eqs.(3.86), (3.108) and (3.112)-(3.118). Corollary to Theorem 2: Let $\left(\alpha_{1}, \alpha_{2}, \alpha_{3}\right) \in D_{\alpha}$. Then, (a)

$$
\lambda_{k+}=\frac{\gamma}{\sin ^{2} \alpha_{k}}>1 \text { and thus } \sqrt{\lambda_{k+}}=\frac{\sqrt{\gamma}}{\sin \alpha_{k}}>1, k=1,2,3
$$

where

$$
\gamma\left(\alpha_{1}, \alpha_{2}, \alpha_{3}\right) \stackrel{\text { def }}{=} \sin ^{2} \alpha_{1}+\sin ^{2} \alpha_{2}+\sin ^{2} \alpha_{3}>0,\left(\alpha_{1}, \alpha_{2}, \alpha_{3}\right) \in D_{\alpha}
$$

is referred to as the shape factor of $\Delta P_{1} P_{2} P_{3}$, hereafter; (b)

$$
\left|W_{k}\right| \stackrel{\text { def }}{=} \text { the determinant of } W_{k}=-\sqrt{\lambda_{k+}}=-\frac{\sqrt{\gamma}}{\sin \alpha_{k}}<-1, k=1,2,3
$$

Thus, for each $k=1,2,3,\left(W_{k}\right)^{-1}$ exists; (c) Eq.(3.118) $\Leftrightarrow$

$$
\hat{\epsilon}_{k}=\left(W_{k}\right)^{-1} \hat{\psi}_{k}, k=1,2,3
$$

and therefore

$$
\text { Eq. }(3.103) \Leftrightarrow \hat{\psi}_{k} \neq\left(\begin{array}{l}
0 \\
0
\end{array}\right) \text {, i.e., }\left(\hat{\psi}_{k}\right)^{t} \hat{\psi}_{k}>0 \text {, for each } k=1,2,3
$$

and $(\mathrm{d})$

$$
\begin{aligned}
& \left(W_{1}\right)^{-1}=\frac{1}{\sqrt{\left(C_{1,2}\right)^{2}+\left(C_{1,3}\right)^{2}}}\left(\begin{array}{cc}
\frac{C_{1,2}}{\sqrt{\lambda_{1+}}} & C_{1,3} \\
\frac{C_{1,3}}{\sqrt{\lambda_{1+}}} & -C_{1,2}
\end{array}\right) \\
& \left(W_{2}\right)^{-1}=\frac{1}{\sqrt{\left(C_{2,3}\right)^{2}+\left(C_{2,1}\right)^{2}}}\left(\begin{array}{cc}
\frac{C_{2,3}}{\sqrt{\lambda_{2+}}} & C_{2,1} \\
\frac{C_{2,1}}{\sqrt{\lambda_{2+}}} & -C_{2,3}
\end{array}\right)
\end{aligned}
$$

and

$$
\left(W_{3}\right)^{-1}=\frac{1}{\sqrt{\left(C_{3,1}\right)^{2}+\left(C_{3,2}\right)^{2}}}\left(\begin{array}{cc}
\frac{C_{3,1}}{\sqrt{\lambda_{3+}}} & C_{3,2} \\
\frac{C_{3,2}}{\sqrt{\lambda_{3+}}} & -C_{3,1}
\end{array}\right)
$$

As a preliminary for a key future development, note that, by using Eqs.(3.93), (3.108), (3.115)-(3.117), (3.138)-(3.140) and (3.143)-(3.145), one has

$$
\begin{aligned}
G_{1} \stackrel{\text { def }}{=} W_{3}\left(J\left(\alpha_{2}, \alpha_{3}, \alpha_{1}\right)\right)^{-1}\left(W_{2}\right)^{-1} & \\
& =\frac{1}{\sqrt{\left(\sin ^{2} \alpha_{2}+\sin ^{2} \alpha_{1}\right)\left(\sin ^{2} \alpha_{3}+\sin ^{2} \alpha_{1}\right)}}\left(\begin{array}{cc}
-\sin \alpha_{2} \cdot \sin \alpha_{3} & -\sqrt{\gamma} \sin \alpha_{1} \\
\sqrt{\gamma} \sin \alpha_{1} & -\sin \alpha_{2} \cdot \sin \alpha_{3}
\end{array}\right)
\end{aligned}
$$




$$
\begin{aligned}
G_{2} \stackrel{\text { def }}{=} W_{1}\left(J\left(\alpha_{3}, \alpha_{1}, \alpha_{2}\right)\right)^{-1}\left(W_{3}\right)^{-1} & \\
& =\frac{1}{\sqrt{\left(\sin ^{2} \alpha_{3}+\sin ^{2} \alpha_{2}\right)\left(\sin ^{2} \alpha_{1}+\sin ^{2} \alpha_{2}\right)}}\left(\begin{array}{cc}
-\sin \alpha_{3} \cdot \sin \alpha_{1} & -\sqrt{\gamma} \sin \alpha_{2} \\
\sqrt{\gamma} \sin \alpha_{2} & -\sin \alpha_{3} \cdot \sin \alpha_{1}
\end{array}\right)
\end{aligned}
$$

and

$$
\begin{aligned}
G_{3} \stackrel{\text { def }}{=} & W_{2}\left(J\left(\alpha_{1}, \alpha_{2}, \alpha_{3}\right)\right)^{-1}\left(W_{1}\right)^{-1} \\
& =\frac{1}{\sqrt{\left(\sin ^{2} \alpha_{1}+\sin ^{2} \alpha_{3}\right)\left(\sin ^{2} \alpha_{2}+\sin ^{2} \alpha_{3}\right)}}\left(\begin{array}{cc}
-\sin \alpha_{1} \cdot \sin \alpha_{2} & -\sqrt{\gamma} \sin \alpha_{3} \\
\sqrt{\gamma} \sin \alpha_{3} & -\sin \alpha_{1} \cdot \sin \alpha_{2}
\end{array}\right)
\end{aligned}
$$

Hereafter, only cases with $\left(\alpha_{1}, \alpha_{2}, \alpha_{3}\right) \in D_{\alpha}$ will be considered.

Next, with the aid of Eqs.(3.146)-(3.148) and the identities:

$$
\begin{aligned}
& \left(\sin ^{2} \alpha_{2}+\sin ^{2} \alpha_{1}\right)\left(\sin ^{2} \alpha_{3}+\sin ^{2} \alpha_{1}\right) \equiv \sin ^{2} \alpha_{2} \cdot \sin ^{2} \alpha_{3}+\gamma \sin ^{2} \alpha_{1} \\
& \left(\sin ^{2} \alpha_{3}+\sin ^{2} \alpha_{2}\right)\left(\sin ^{2} \alpha_{1}+\sin ^{2} \alpha_{2}\right) \equiv \sin ^{2} \alpha_{3} \cdot \sin ^{2} \alpha_{1}+\gamma \sin ^{2} \alpha_{2}
\end{aligned}
$$

and

$$
\left(\sin ^{2} \alpha_{1}+\sin ^{2} \alpha_{3}\right)\left(\sin ^{2} \alpha_{2}+\sin ^{2} \alpha_{3}\right) \equiv \sin ^{2} \alpha_{1} \cdot \sin ^{2} \alpha_{2}+\gamma \sin ^{2} \alpha_{3}
$$

One has

$$
\left(G_{k}\right)^{t}\left(G_{k}\right)=I_{2}, \quad k=1,2,3
$$

Thus, for each $k=1,2,3, G_{k}$ is a real orthogonal matrix [Ref.9, p.126], i.e.,

$$
\left(G_{k}\right)^{-1}=\left(G_{k}\right)^{t}, \quad k=1,2,3
$$

Next, by using Eqs.(3.91)(a)-(3.91)(c), (3.118), (3.141) and (3.146)-(3.148) one can show that

$$
\hat{\psi}_{2}=G_{3} \hat{\psi}_{1}, \hat{\psi}_{3}=G_{1} \hat{\psi}_{2} \text { and } \hat{\psi}_{1}=G_{2} \hat{\psi}_{3}
$$

Proof for Eq.(3.154): By using Eqs.(3.118), (3.91)(a), (3.141) and (3.148) one has

$$
\hat{\psi}_{2}=W_{2} \hat{\epsilon}_{2}=W_{2}\left(J\left(\alpha_{1}, \alpha_{2}, \alpha_{3}\right)\right)^{-1} \hat{\epsilon}_{1}=W_{2}\left(J\left(\alpha_{1}, \alpha_{2}, \alpha_{3}\right)\right)^{-1}\left(W_{1}\right)^{-1} \hat{\psi}_{1}=G_{3} \hat{\psi}_{1}
$$

i.e., the first part of Eq.(3.154) has been proved. Similary, by using Eqs.(3.118), (3.91)(b), (3.141), (3.146) and (3.147), one can prove the second and third parts of Eq.(3.154). QED.

Note that, by using Eq.(3.153), it can be shown that Eq.(3.154) $\Leftrightarrow$

$$
\hat{\psi}_{1}=\left(G_{3}\right)^{-1} \hat{\psi}_{2}=\left(G_{3}\right)^{t} \hat{\psi}_{2}, \hat{\psi}_{2}=\left(G_{1}\right)^{-1} \hat{\psi}_{3}=\left(G_{1}\right)^{t} \hat{\psi}_{3} \text { and } \hat{\psi}_{3}=\left(G_{2}\right)^{-1} \hat{\psi}_{1}=\left(G_{2}\right)^{t} \hat{\psi}_{1}
$$

Also, by using Eqs.(3.106), (3.107) and (3.142), one concludes that, the current basic assumption that $\left(\epsilon_{1}, \epsilon_{2}, \epsilon_{3}\right) \in \Lambda^{\prime}\left(\alpha_{1}, \alpha_{2}, \alpha_{3}\right) \Leftrightarrow$

$$
\hat{\psi}_{k} \neq\left(\begin{array}{l}
0 \\
0
\end{array}\right) \text {, i.e., }\left(\hat{\psi}_{k}\right)^{t} \hat{\psi}_{k}>0 \text { for each } k=1,2,3 \text { and each }\left(\alpha_{1}, \alpha_{2}, \alpha_{3}\right) \in D_{\alpha}
$$

At this juncture, also note that a result of Eq.(3.121) is

$$
\left(\hat{\psi}_{1}\right)^{t} \hat{\psi}_{1} \equiv\left(\hat{\psi}_{2}\right)^{t} \hat{\psi}_{2} \equiv\left(\hat{\psi}_{3}\right)^{t} \hat{\psi}_{3}
$$

which can also be proved directly using Eqs.(3.153) and (3.154). Recall that Eqs.(3.153) and (3.154) are derived using (i) the relations given in Eqs.(3.91)(a)-(3.91)(c) which are derived from Eq.(3.89), (ii) the definition of Eq.(3.118) and its inverse relation Eq.(3.141), and (iii) the definitions $G_{1}, G_{2}$ and $G_{3}$ given in Eqs.(3.146)-(3.148). In other words, Eq.(3.157) represents a set of identity relations which follow directly 
from Eq.(3.89). As such, with the aid of the fact that $\left(\epsilon_{1}, \epsilon_{2}, \epsilon_{3}\right) \in \Lambda^{\prime}\left(\alpha_{1}, \alpha_{2}, \alpha_{3}\right) \Leftrightarrow$ Eq.(3.156), Eq.(3.121) $\mathrm{c}$ an be replaced by a simpler equivalent form, i.e.,

$$
\left(\epsilon^{*}\right)^{2}=\left(\hat{\psi}_{1}\right)^{t} \hat{\psi}_{1} \text { for each } \hat{\psi}_{1} \neq\left(\begin{array}{l}
0 \\
0
\end{array}\right) \text {, i.e., }\left(\hat{\psi}_{1}\right)^{t} \hat{\psi}_{1}>0
$$

Next, by using Eqs.(3.55) and (3.141), one has

$$
\frac{3}{2}(|\overrightarrow{\nabla \nu}|)^{2}=\frac{3}{2}\left[\left(W_{k}\right)^{-1} \hat{\psi}_{k}\right]^{t} S\left(\alpha_{k}\right)\left(W_{k}\right)^{-1} \hat{\psi}_{k}=(\hat{\psi})^{t} H_{k} \hat{\psi}, k=1,2,3
$$

where

$$
H_{k} \stackrel{\text { def }}{=} \frac{3}{2}\left[\left(W_{k}\right)^{-1}\right]^{t} S\left(\alpha_{k}\right)\left(W_{k}\right)^{-1}, k=1,2,3 \text { and }\left(\alpha_{1}, \alpha_{2}, \alpha_{3}\right) \in D_{\alpha}
$$

According to Eq.(3.53), for each $k=1,2,3, S\left(\alpha_{k}\right)$ is a real symmetric matrix. In turn, Eq.(3.160) $\Rightarrow$ for each $k=1,2,3, H_{k}$ is also a real symmetric matrix. As such, for each $k=1,2,3$, the eigenvalues of $H_{k}$ are all real [Ref.9, p.222]. Moreover, as will be shown, the matrices $H_{1}, H_{2}$ and $H_{3}$ are similar [Ref.9, p.232], i.e., they are related by the following similarity transformations:

$$
H_{1}=\left(G_{3}\right)^{-1} H_{2} G_{3}, H_{2}=\left(G_{1}\right)^{-1} H_{3} G_{1} \text { and } H_{3}=\left(G_{2}\right)^{-1} H_{1} G_{2}
$$

where $G_{1}, G_{2}$ and $G_{3}$ are the matrices defined in Eqs.(3.146)-(3.148), respectively. According to a matrix theorem [Ref.9, p.232], similar matrices such as $H_{1}, H_{2}$ and $H_{3}$ have the same eigenvalues with the same multiplicities. In the following, first we prove Eq.(3.161).

Proof of Eq.(3.161): By using Eqs.(3.160), (3.94)(a), (3.148) and (3.153) one has

$$
\begin{aligned}
H_{1} & =\frac{3}{2}\left[\left(W_{1}\right)^{-1}\right]^{t} S\left(\alpha_{1}\right)\left(W_{1}\right)^{-1}=\frac{3}{2}\left[\left(W_{1}\right)^{-1}\right]^{t}\left[\left(J\left(\alpha_{1}, \alpha_{2}, \alpha_{3}\right)\right)^{-1}\right]^{t} S\left(\alpha_{2}\right)\left(J\left(\alpha_{1}, \alpha_{2}, \alpha_{3}\right)\right)^{-1}\left(W_{1}\right)^{-1} \\
& =\frac{3}{2}\left[\left(W_{1}\right)^{-1}\right]^{t}\left[\left(J\left(\alpha_{1}, \alpha_{2}, \alpha_{3}\right)\right)^{-1}\right]^{t}\left(W_{2}\right)^{t}\left[\left(W_{2}\right)^{-1}\right]^{t} S\left(\alpha_{2}\right)\left(W_{2}\right)^{-1} W_{2}\left(J\left(\alpha_{1}, \alpha_{2}, \alpha_{3}\right)\right)^{-1}\left(W_{1}\right)^{-1} \\
& =\left[W_{2}\left(J\left(\alpha_{1}, \alpha_{2}, \alpha_{3}\right)\right)^{-1}\left(W_{1}\right)^{-1}\right]^{t} \frac{3}{2}\left[\left(W_{2}\right)^{-1}\right]^{t} S\left(\alpha_{2}\right)\left(W_{2}\right)^{-1}\left[W_{2}\left(J\left(\alpha_{1}, \alpha_{2}, \alpha_{3}\right)\right)^{-1}\left(W_{1}\right)^{-1}\right] \\
& =\left(G_{3}\right)^{t} H_{2} G_{3}=\left(G_{3}\right)^{-1} H_{2} G_{3}
\end{aligned}
$$

i.e., the first part of Eq.(3.161) has been proved. Next, with the aid of Eqs.(3.160), (3.94)(b), (3.146) and (3.153), it can be shown that the new version of Eq.(3.162) after it undergoes the index permutation of Eq.(3.44) is also valid, i.e., the second part of Eq.(3.161) is also valid. Similarly, once can also prove the last part. QED.

Obviously, Eq.(3.161) can be cast in the following equivalent form:

$$
H_{2}=G_{3} H_{1}\left(G_{3}\right)^{-1}, H_{3}=G_{1} H_{2}\left(G_{1}\right)^{-1} \text { and } H_{1}=G_{2} H_{3}\left(G_{2}\right)^{-1}
$$

Moreover, by using Eqs.(3.153), (3.154) and (3.163), one can show that

$$
\left(\hat{\psi}_{1}\right)^{t} H_{1} \hat{\psi}_{1} \equiv\left(\hat{\psi}_{2}\right)^{t} H_{2} \hat{\psi}_{2} \equiv\left(\hat{\psi}_{3}\right)^{t} H_{3} \hat{\psi}_{3}
$$

In turn, with the aid of Eq.(3.164), Eq.(3.159) can be replaced by a simpler form

$$
\frac{3}{2}(|\overrightarrow{\nabla \nu}|)^{2}=\left(\hat{\psi}_{1}\right)^{t} H_{1} \hat{\psi}_{1}
$$

To study the eigenvalues of $H_{1}$, note that, with the aid of Eqs.(3.108) and (3.138), Eq.(3.143) $\Rightarrow$

$$
\left(W_{1}\right)^{-1}=\frac{\sin \alpha_{1}}{\sqrt{\sin ^{2} \alpha_{2}+\sin ^{2} \alpha_{3}}}\left(\begin{array}{cc}
\frac{\sin \alpha_{2}}{\sqrt{\gamma}} & \frac{\sin \alpha_{3}}{\sin \alpha_{1}} \\
\frac{\sin \alpha_{3}}{\sqrt{\gamma}} & -\frac{\sin \alpha_{2}}{\sin \alpha_{1}}
\end{array}\right),\left(\alpha_{1}, \alpha_{2}, \alpha_{3}\right) \in D_{\alpha}
$$

In turn, by substituting Eq.(3.53), (3.139) and (3.166) into Eq.(3.160), one has

$$
H_{1}=\frac{3}{2}\left(\begin{array}{ll}
d_{1} & e_{1} \\
e_{1} & f_{1}
\end{array}\right),\left(\alpha_{1}, \alpha_{2}, \alpha_{3}\right) \in D_{\alpha}
$$


where,

$$
\begin{gathered}
d_{1} \stackrel{\text { def }}{=} \frac{\sin ^{2} \alpha_{2}+\sin ^{2} \alpha_{3}+2\left(\cos \alpha_{1}\right)\left(\sin \alpha_{2}\right)\left(\sin \alpha_{3}\right)}{\left(\sin ^{2} \alpha_{2}+\sin ^{2} \alpha_{3}\right)\left(\sin ^{2} \alpha_{1}+\sin ^{2} \alpha_{2}+\sin ^{2} \alpha_{3}\right)}, \quad\left(\alpha_{1}, \alpha_{2}, \alpha_{3}\right) \in D_{\alpha} \\
e_{1} \stackrel{\text { def }}{=} \frac{\cos \alpha_{1}\left(\sin ^{2} \alpha_{3}-\sin ^{2} \alpha_{2}\right)}{\sin \alpha_{1}\left(\sin ^{2} \alpha_{2}+\sin ^{2} \alpha_{3}\right) \sqrt{\left(\sin ^{2} \alpha_{1}+\sin ^{2} \alpha_{2}+\sin ^{2} \alpha_{3}\right)}}, \quad\left(\alpha_{1}, \alpha_{2}, \alpha_{3}\right) \in D_{\alpha}
\end{gathered}
$$

and

$$
f_{1} \stackrel{\text { def }}{=} \frac{\sin ^{2} \alpha_{2}+\sin ^{2} \alpha_{3}-2\left(\cos \alpha_{1}\right)\left(\sin \alpha_{2}\right)\left(\sin \alpha_{3}\right)}{\sin ^{2} \alpha_{1}\left(\sin ^{2} \alpha_{2}+\sin ^{2} \alpha_{3}\right)}, \quad\left(\alpha_{1}, \alpha_{2}, \alpha_{3}\right) \in D_{\alpha}
$$

By definition, an eigenvalue $\sigma$ of $H_{1}$ is a root of the equation:

$$
\text { the determinant of the matrix }\left(H_{1}-\sigma I_{2}\right)=0
$$

Substituting Eq.(3.167) into Eq.(3.171), one has

$$
\sigma^{2}-\frac{3}{2}\left(d_{1}+f_{1}\right) \sigma+\frac{9}{4}\left[d_{1} \cdot f_{1}-\left(e_{1}\right)^{2}\right]=0
$$

Thus,

$$
\sigma=\sigma_{1+} \text { or } \sigma=\sigma_{1-}
$$

where

$$
\sigma_{1 \pm} \stackrel{\text { def }}{=} \frac{3}{4}\left\{d_{1}+f_{1} \pm \sqrt{\left(d_{1}+f_{1}\right)^{2}-4\left[d_{1} \cdot f_{1}-\left(e_{1}\right)^{2}\right]}\right\}
$$

Note that, because

$$
\left(d_{1}+f_{1}\right)^{2}-4\left[d_{1} \cdot f_{1}-\left(e_{1}\right)^{2}\right]=\left(d_{1}-f_{1}\right)^{2}+4\left(e_{1}\right)^{2} \geq 0
$$

the expression under the radical sign in Eq.(3.174) is non-negative, Thus, for any $\left(\alpha_{1}, \alpha_{2}, \alpha_{3}\right) \in D_{\alpha}$, the eigne values $\sigma_{1+}$ and $\sigma_{1-}$ of $H_{1}$ are real always, a fact consistent with the fact that the eigenvalues of the real symmetric matrix $H_{1}$ must be real always [Ref.9, p. 222].

Next, with the aid of Eqs.(3.139) and (3.168)-(3.170), one has (i)

$$
\begin{aligned}
d_{1} \cdot f_{1}-\left(e_{1}\right)^{2} & =\frac{\left(\sin ^{2} \alpha_{2}+\sin ^{2} \alpha_{3}\right)^{2}-4\left(\cos ^{2} \alpha_{1}\right)\left(\sin ^{2} \alpha_{2}\right)\left(\sin ^{2} \alpha_{3}\right)-\left(\cos ^{2} \alpha_{1}\right)\left(\sin ^{2} \alpha_{3}-\sin ^{2} \alpha_{2}\right)^{2}}{\left(\sin ^{2} \alpha_{1}\right)\left(\sin ^{2} \alpha_{2}+\sin ^{2} \alpha_{3}\right)^{2}\left(\sin ^{2} \alpha_{1}+\sin ^{2} \alpha_{2}+\sin ^{2} \alpha_{3}\right)} \\
& =\frac{\left(\sin ^{2} \alpha_{2}+\sin ^{2} \alpha_{3}\right)^{2}-\left(\cos ^{2} \alpha_{1}\right)\left(\sin ^{2} \alpha_{2}+\sin ^{2} \alpha_{3}\right)^{2}}{\left(\sin ^{2} \alpha_{1}\right)\left(\sin ^{2} \alpha_{2}+\sin ^{2} \alpha_{3}\right)^{2}\left(\sin ^{2} \alpha_{1}+\sin ^{2} \alpha_{2}+\sin ^{2} \alpha_{3}\right)} \\
& =\frac{\left(\sin ^{2} \alpha_{1}\right)\left(\sin ^{2} \alpha_{2}+\sin ^{2} \alpha_{3}\right)^{2}}{\left(\sin ^{2} \alpha_{1}\right)\left(\sin ^{2} \alpha_{2}+\sin ^{2} \alpha_{3}\right)^{2}\left(\sin ^{2} \alpha_{1}+\sin ^{2} \alpha_{2}+\sin ^{2} \alpha_{3}\right)} \\
& =\frac{1}{\left(\sin ^{2} \alpha_{1}+\sin ^{2} \alpha_{2}+\sin ^{2} \alpha_{3}\right)}=\frac{1}{\gamma}, \quad\left(\alpha_{1}, \alpha_{2}, \alpha_{3}\right) \in D_{\alpha}
\end{aligned}
$$

and (ii)

$$
d_{1}+f_{1}=\frac{q_{1}}{\gamma\left(\sin ^{2} \alpha_{1}\right)\left(\sin ^{2} \alpha_{2}+\sin ^{2} \alpha_{3}\right)}
$$

where.

$$
\begin{aligned}
q_{1} & \stackrel{\text { def }}{=}\left(\sin ^{2} \alpha_{1}\right)\left[\sin ^{2} \alpha_{2}+\sin ^{2} \alpha_{3}+2\left(\cos \alpha_{1}\right)\left(\sin \alpha_{2}\right)\left(\sin \alpha_{3}\right)\right] \\
& +\left(\sin ^{2} \alpha_{1}+\sin ^{2} \alpha_{2}+\sin ^{2} \alpha_{3}\right)\left[\sin ^{2} \alpha_{2}+\sin ^{2} \alpha_{3}-2\left(\cos \alpha_{1}\right)\left(\sin \alpha_{2}\right)\left(\sin \alpha_{3}\right)\right] \\
& =\left(\sin ^{2} \alpha_{1}\right)\left(\sin ^{2} \alpha_{2}+\sin ^{2} \alpha_{3}\right)+2\left(\sin ^{2} \alpha_{1}\right)\left(\cos \alpha_{1}\right)\left(\sin \alpha_{2}\right)\left(\sin \alpha_{3}\right)+ \\
& \left(\sin ^{2} \alpha_{1}+\sin ^{2} \alpha_{2}+\sin ^{2} \alpha_{3}\right)\left(\sin ^{2} \alpha_{2}+\sin ^{2} \alpha_{3}\right)-2\left(\sin ^{2} \alpha_{1}\right)\left(\cos \alpha_{1}\right)\left(\sin \alpha_{2}\right)\left(\sin \alpha_{3}\right) \\
& -2\left(\sin ^{2} \alpha_{2}+\sin ^{2} \alpha_{3}\right)\left(\cos \alpha_{1}\right)\left(\sin \alpha_{2}\right)\left(\sin \alpha_{3}\right) \\
& =\left(\sin ^{2} \alpha_{2}+\sin ^{2} \alpha_{3}\right)\left[2 \sin ^{2} \alpha_{1}+\sin ^{2} \alpha_{2}+\sin ^{2} \alpha_{3}-2\left(\cos \alpha_{1}\right)\left(\sin \alpha_{2}\right)\left(\sin \alpha_{3}\right)\right]
\end{aligned}
$$

To simplify Eq.(3.178), next we will prove the identity:

$$
\sin ^{2} \alpha_{2}+\sin ^{2} \alpha_{3}-2\left(\cos \alpha_{1}\right)\left(\sin \alpha_{2}\right)\left(\sin \alpha_{3}\right)=\sin ^{2} \alpha_{1},\left(\alpha_{1}, \alpha_{2}, \alpha_{3}\right) \in D_{\alpha}
$$


Proof of Eq.(3.179): By using Eq.(3.24) and Fig.3(a), the law of cosines can be expressed as

$$
\left(s_{1,3}\right)^{2}+\left(s_{1,2}\right)^{2}-2\left(\cos \alpha_{1}\right) s_{1,3} \cdot s_{1,2}=\left(s_{2,3}\right)^{2}, \quad\left(\alpha_{1}, \alpha_{2}, \alpha_{3}\right) \in D_{\alpha}
$$

Because $s_{3,1}=s_{1,3}$ and $\sigma>0$, Eq.(3.179) now follows directly from Eqs.(3.88) and (3.180). Eq.(3.179) can also be proved directly without invoking the law of cosines. Because, $\left(\alpha_{1}, \alpha_{2}, \alpha_{3}\right) \in D_{\alpha} \Rightarrow \alpha_{1}=\pi-\alpha_{2}-\alpha_{3}$, we have

$$
\begin{aligned}
\sin ^{2} \alpha_{1} & =\sin ^{2}\left(\alpha_{2}+\alpha_{3}\right)=\left(\sin \alpha_{2} \cos \alpha_{3}+\sin \alpha_{3} \cos \alpha_{2}\right)^{2} \\
& =\left(\sin ^{2} \alpha_{2}\right)\left(\cos ^{2} \alpha_{3}\right)+\left(\sin ^{2} \alpha_{3}\right)\left(\cos ^{2} \alpha_{2}\right)+2\left(\sin \alpha_{2}\right)\left(\sin \alpha_{3}\right)\left(\cos \alpha_{2}\right)\left(\cos \alpha_{3}\right) \\
& =\left(\sin ^{2} \alpha_{2}\right)\left(1-\sin ^{2} \alpha_{3}\right)+\left(\sin ^{2} \alpha_{3}\right)\left(1-\sin ^{2} \alpha_{2}\right)+2\left(\sin \alpha_{2}\right)\left(\sin \alpha_{3}\right)\left(\cos \alpha_{2}\right)\left(\cos \alpha_{3}\right) \\
& =\sin ^{2} \alpha_{2}+\sin ^{2} \alpha_{3}+2\left(\sin \alpha_{2}\right)\left(\sin \alpha_{3}\right) \cos \left(\alpha_{2}+\alpha_{3}\right)=\sin ^{2} \alpha_{2}+\sin ^{2} \alpha_{3}-2\left(\cos \alpha_{1}\right)\left(\sin \alpha_{2}\right)\left(\sin \alpha_{3}\right)
\end{aligned}
$$

i.e., Eq.(3.179) has been proved. QED. With the aid of Eq.(3.179), Eq.(3.178) now implies that

$$
q_{1}=3\left(\sin ^{2} \alpha_{1}\right)\left(\sin ^{2} \alpha_{2}+\sin ^{2} \alpha_{3}\right)
$$

In turn, by substituting Eq.(3.181) into Eq.(3.177), one has

$$
d_{1}+f_{1}=\frac{3}{\gamma}, \quad\left(\alpha_{1}, \alpha_{2}, \alpha_{3}\right) \in D_{\alpha}
$$

Next, with the aid of Eqs.(3.176) and (3.182), Eqs.(3.174) and (3.175) imply that, for any given $\left(\alpha_{1}, \alpha_{2}, \alpha_{3}\right) \in D_{\alpha},(\mathrm{i})$

$$
\sigma_{1 \pm}\left(\alpha_{1}, \alpha_{2}, \alpha_{3}\right)=\sigma_{ \pm}(\gamma) \stackrel{\text { def }}{=} \frac{3}{4 \gamma}[3 \pm 2 \sqrt{(9 / 4)-\gamma}],\left(\alpha_{1}, \alpha_{2}, \alpha_{3}\right) \in D_{\alpha}
$$

and (ii)

$$
\frac{4}{\gamma^{2}}\left(\frac{9}{4}-\gamma\right)=\left(d_{1}-f_{1}\right)^{2}+4\left(e_{1}\right)^{2} \geq 0, \quad\left(\alpha_{1}, \alpha_{2}, \alpha_{3}\right) \in D_{\alpha}
$$

In turn, Eqs.(3.139) and (3.184) $\Rightarrow$

$$
0<\gamma \leq \frac{9}{4}, \quad\left(\alpha_{1}, \alpha_{2}, \alpha_{3}\right) \in D_{\alpha}
$$

Note that, it was shown earlier that $H_{1}, H_{2}$ and $H_{3}$ are similar and thus they must have the same eigenvalues. As such, Eq. (3.183) implies that $\sigma_{+}(\gamma)$ and $\sigma_{-}(\gamma)$ must be invariant under the index permutation of Eq. (3.44) - A fact that leads to the expectation that they must be a function of parameters (such as $\gamma$ ) which are invariant under the permutation.

Moreover, it is shown in Appendix B that, by using Eq.(3.139), one can prove directly that, (i) Eq.(3.185) represents the range of $\gamma$ over $D_{\alpha}$; (ii)

$$
\gamma=\frac{9}{4} \Leftrightarrow \alpha_{1}=\alpha_{2}=\alpha_{3}=\frac{\pi}{3} \text { if }\left(\alpha_{1}, \alpha_{2}, \alpha_{3}\right) \in D_{\alpha}
$$

i.e., the shape factor $\gamma$ reaches its maximal value $\frac{9}{4}$ if and only if $\Delta P_{1} P_{2} P_{3}$ is equilateral; and (iii) for the case $\left(\alpha_{1}, \alpha_{2}, \alpha_{3}\right) \in D_{\alpha}$

$$
\begin{aligned}
\gamma \rightarrow 0^{+} \Leftrightarrow \min \left\{\alpha_{1}, \alpha_{2}, \alpha_{3}\right\} & \rightarrow 0^{+} \\
\text {and } \max \left\{\alpha_{1}, \alpha_{2}, \alpha_{3}\right\} & \rightarrow \pi^{-}
\end{aligned}
$$

i.e., for the case $\left(\alpha_{1}, \alpha_{2}, \alpha_{3}\right) \in D_{\alpha}, \gamma \rightarrow 0^{+} \Leftrightarrow$ the value of the largest internal angle approaches $\pi^{-}$while those of the other two approach $0^{+}$.

Moreover, by using Eqs.(3.183), (3.186) and (3.167)-(3.170), one can show that, for the case $\left(\alpha_{1}, \alpha_{2}, \alpha_{3}\right) \in$ $D_{\alpha},(\mathrm{i})$

$$
\sigma_{+}(\gamma)=\sigma_{-}(\gamma) \Leftrightarrow \gamma=\frac{9}{4} \Leftrightarrow \alpha_{1}=\alpha_{2}=\alpha_{3}=\frac{\pi}{3} \Leftrightarrow H_{1}=I_{2} \text { for the case }\left(\alpha_{1}, \alpha_{2}, \alpha_{3}\right) \in D_{\alpha}
$$


(ii)

$$
\sigma_{ \pm}\left(\frac{9}{4}\right)=1 \text { and } \lim _{\gamma \rightarrow 0^{+}} \sigma_{+}(\gamma)=+\infty
$$

(iii)

$$
\frac{d \sigma_{+}(\gamma)}{d \gamma}\left\{\begin{array}{l}
\text { is undefined at } \gamma=\frac{9}{4} \\
=-\frac{3}{8}\left[\frac{9-2 \gamma+6 \sqrt{(9 / 4)-\gamma}}{\left(\gamma^{2}\right) \sqrt{(9 / 4)-\gamma}}\right] \text { if } 0<\gamma<\frac{9}{4}
\end{array}\right.
$$

and (iv)

$$
\frac{d \sigma_{-}(\gamma)}{d \gamma}\left\{\begin{array}{l}
\text { is undefined at } \gamma=\frac{9}{4} \\
=\frac{3}{8}\left[\frac{9-2 \gamma-6 \sqrt{(9 / 4)-\gamma}}{\left(\gamma^{2}\right) \sqrt{(9 / 4)-\gamma}}\right] \text { if } 0<\gamma<\frac{9}{4}
\end{array}\right.
$$

Because,

$$
9-2 \gamma+6 \sqrt{(9 / 4)-\gamma}=4\left(\frac{9}{4}-\gamma\right)+2 \gamma+6 \sqrt{(9 / 4)-\gamma}>0,0<\gamma<\frac{9}{4}
$$

Eq. $(3.190) \Rightarrow$

$$
\frac{d \sigma_{+}(\gamma)}{d \gamma}<0 \quad \text { if } 0<\gamma<\frac{9}{4}
$$

On the other hand, because

$$
\begin{array}{r}
9-2 \gamma-6 \sqrt{(9 / 4)-\gamma}=\frac{(9-2 \gamma-6 \sqrt{(9 / 4)-\gamma})(9-2 \gamma+6 \sqrt{(9 / 4)-\gamma})}{9-2 \gamma+6 \sqrt{(9 / 4)-\gamma}} \\
=\frac{4 \gamma^{2}}{(9-2 \gamma+6 \sqrt{(9 / 4)-\gamma})}>0 \text { if } 0<\gamma<\frac{9}{4}
\end{array}
$$

Eq.(3.191) $\Rightarrow$

$$
\frac{d \sigma_{-}(\gamma)}{d \gamma}>0 \text { if } 0<\gamma<\frac{9}{4}
$$

To study the behavior of $\sigma_{-}(\gamma)$ in the limit of $\gamma \rightarrow 0^{+}$, note that (i)

$$
\lim _{\gamma \rightarrow 0^{+}}(3-2 \sqrt{(9 / 4)-\gamma})=0 \text { and } \lim _{\gamma \rightarrow 0^{+}} \gamma=0
$$

and (ii)

$$
\frac{d[3-2 \sqrt{(9 / 4)-\gamma}]}{d \gamma}=\frac{1}{\sqrt{(9 / 4)-\gamma}} \quad \text { and } \quad \frac{d \gamma}{d \gamma}=1, \quad 0<\gamma<\frac{9}{4}
$$

Then, with the aid of Eqs.(3.196) and (3.197), an application of L'Hospital rule over the function $\sigma_{-}(\gamma)$ defined in Eq.(3.183) $\Rightarrow$

$$
\lim _{\gamma \rightarrow 0^{+}} \sigma_{-}(\gamma)=\frac{3}{4} \lim _{\gamma \rightarrow 0^{+}}\left(\frac{1}{\sqrt{(9 / 4)-\gamma}}\right)=\frac{3}{4} \times \frac{2}{3}=\frac{1}{2}
$$

By using Eqs.(3.189)-(3.191), (3.193), (3.195) and (3.198), one concludes that: as the value of $\gamma$ decreases from $\frac{9}{4}$ to $0^{+}$, (i) the value of $\sigma_{+}(\gamma)$ increases monotonically from 1 to $+\infty$; and (ii) the value of $\sigma_{-}(\gamma)$ decreases monotonically from 1 towards the limit value $1 / 2$. Thus, one has (i)

$$
\sigma_{-}(\gamma)=1 \Leftrightarrow \sigma_{+}(\gamma)=1 \Leftrightarrow \gamma=\frac{9}{4}
$$


(ii)

$$
\frac{1}{2}<\sigma_{-}(\gamma)<1<\sigma_{+}(\gamma) \quad \text { if } 0<\gamma<\frac{9}{4}
$$

and (iii)

$$
\sigma_{-}(\gamma) \rightarrow\left(\frac{1}{2}\right)^{+} \Leftrightarrow \sigma_{+}(\gamma) \rightarrow+\infty \Leftrightarrow \gamma \rightarrow 0^{+}
$$

Note that Eq.(3.139) $\Rightarrow$

$$
\gamma=2 \text { if } \max \left\{\alpha_{1}, \alpha_{2}, \alpha_{3}\right\}=\frac{\pi}{2} \text { and }\left(\alpha_{1}, \alpha_{2}, \alpha_{3}\right) \in D_{\alpha}
$$

Thus, with the aid of Eq.(3.183), one concludes that

$$
\begin{gathered}
\sigma_{+}(\gamma)=\sigma_{+}(2)=\frac{3}{2} \text { and } \sigma_{-}(\gamma)=\sigma_{-}(2)=\frac{3}{4} \\
\text { if } \max \left\{\alpha_{1}, \alpha_{2}, \alpha_{3}\right\}=\frac{\pi}{2} \text { and }\left(\alpha_{1}, \alpha_{2}, \alpha_{3}\right) \in D_{\alpha}
\end{gathered}
$$

At this juncture, note that Eqs.(3.139), (3.183), (3.185)-(3.187) and (3.199)-(3.201) imply that: (i)

$$
\sigma_{1-}\left(\frac{\pi}{3}, \frac{\pi}{3}, \frac{\pi}{3}\right)=\sigma_{1+}\left(\frac{\pi}{3}, \frac{\pi}{3}, \frac{\pi}{3}\right)=\sigma_{-}\left(\frac{9}{4}\right)=\sigma_{+}\left(\frac{9}{4}\right)=1
$$

(ii)

$$
\begin{array}{r}
\frac{1}{2}<\sigma_{1-}\left(\alpha_{1}, \alpha_{2}, \alpha_{3}\right)=\sigma_{-}(\gamma)<1<\sigma_{1+}\left(\alpha_{1}, \alpha_{2}, \alpha_{3}\right)=\sigma_{+}(\gamma) \\
\text { if } \left.\left(\alpha_{1}, \alpha_{2}, \alpha_{3}\right) \neq\left(\frac{\pi}{3}, \frac{\pi}{3}, \frac{\pi}{3}\right) \text { (i.e., } 0<\gamma<\frac{9}{4}\right) \text { and }\left(\alpha_{1}, \alpha_{2}, \alpha_{3}\right) \in D_{\alpha}
\end{array}
$$

and (iii)

$$
\begin{array}{r}
\sigma_{1-}\left(\alpha_{1}, \alpha_{2}, \alpha_{3}\right)=\sigma_{-}(\gamma) \rightarrow\left(\frac{1}{2}\right)^{-} \Leftrightarrow \sigma_{1+}\left(\alpha_{1}, \alpha_{2}, \alpha_{3}\right)=\sigma_{+}(\gamma) \rightarrow+\infty \Leftrightarrow \\
\gamma \rightarrow 0^{+} \Leftrightarrow \min \left\{\alpha_{1}, \alpha_{2}, \alpha_{3}\right\} \rightarrow 0^{+} \text {and } \max \left\{\alpha_{1}, \alpha_{2}, \alpha_{3}\right\} \rightarrow \pi^{-}, \\
\text {if }\left(\alpha_{1}, \alpha_{2}, \alpha_{3}\right) \in D_{\alpha}
\end{array}
$$

However, by using the results presented above and the fact that an $n \times n$ real symmetric matrix possesses a set of $n$ linearly independent real eigenvectors associated with its exclusive real eigenvalues [Ref.8, p.306], one concludes that, for each $\left(\alpha_{1}, \alpha_{2}, \alpha_{3}\right) \in D_{\alpha}$, there exist two linearly independent eigenvectors $\hat{\psi}_{1-}(\gamma)$ and $\hat{\psi}_{1+}(\gamma)$ such that

$$
\begin{array}{r}
H_{1} \hat{\psi}_{1-}(\gamma)=\sigma_{-}(\gamma) \hat{\psi}_{1-}(\gamma) \quad \text { and } \quad H_{1} \hat{\psi}_{1+}(\gamma)=\sigma_{+}(\gamma) \hat{\psi}_{1+}(\gamma) \\
\text { for each } \gamma \text { with } 0<\gamma \leq \frac{9}{4} \text { and each }\left(\alpha_{1}, \alpha_{2}, \alpha_{3}\right) \in D_{\alpha}
\end{array}
$$

With the above preliminaries, we are ready to tackle the central question that motivates the current study. First, note that, by using Eqs.(3.51) and (3.105)-(3.107)

$$
R \stackrel{\text { def }}{=} \sqrt{\frac{3}{2}} \frac{|\overrightarrow{\Delta \nu}|}{\epsilon^{*}}=\sqrt{\frac{\left[\left(\Delta \nu_{x}\right)^{2}+\left(\Delta \nu_{y}\right)^{2}\right] / 2}{\left[\left(\epsilon_{1}\right)^{2}+\left(\epsilon_{2}\right)^{2}+\left(\epsilon_{3}\right)^{2}\right] / 3}} \geq 0,\left(\epsilon_{1}, \epsilon_{2}, \epsilon_{3}\right) \in \Lambda^{\prime}\left(\alpha_{1}, \alpha_{2}, \alpha_{3}\right)
$$

By definition, $R$ is the square root of the ratio of the two simple averages, i.e., the simple average of $\left(\Delta \nu_{x}\right)^{2}$ and $\left(\Delta \nu_{y}\right)^{2}$, and that of $\left(\epsilon_{1}\right)^{2},\left(\epsilon_{2}\right)^{2}$ and $\left(\epsilon_{3}\right)^{2}$. As such $R$ is a measure of the relative magnitudes of the error norms $\epsilon^{*} / \sqrt{3}$ and $|\overrightarrow{\Delta \nu}| / \sqrt{2}$. According to Eqs.(3.35)-(3.37), (3.40) and (3.105), $\epsilon^{*} / \sqrt{3}$ is an error norm associated with the numerical errors of the directional derivatives of the scalar function $\phi$ evaluated along the three sides of $\Delta P_{1} P_{2} P_{3}$. On the other hand, according to Eqs.(3.45),(3.46) and (3.51), $|\overrightarrow{\Delta \nu}| / \sqrt{2}$ is an error norm associated with the numerical error of the constant gradient vector $\phi$ on the plane $\Gamma$ in the $x-y-\phi$ space, defined by Eqs.(3.5) and (3.6). As such $R$ is a measure of how large the error norm associated with the gradient vector $\phi$ on 
$\Gamma$ is amplified from that associated with the directional derivatives of $\phi$ evaluated along the three sides of $\Delta P_{1} P_{2} P_{3}$. Thus, by its definition, $R$ represents an error amplification factor.

Numerical experiments reveal that the value of $R$ could (but not necessary) become very large if $\Delta P_{1} P_{2} P_{3}$ has a large aspect ratio. In the following, we will provide a mathematically rigorous explanation of this pitfall and also a way to avoid it even in a case in which use of a triangular grid with a large aspect ratio is unavoidable.

To proceed, note that it was shown earlier that the assumption $\left(\epsilon_{1}, \epsilon_{2}, \epsilon_{3}\right) \in \Lambda^{\prime}\left(\alpha_{1}, \alpha_{2}, \alpha_{3}\right) \Leftrightarrow$ Eq.(3.156). Thus, by combining Eqs.(3.158), (3.165) and (3.208), one has

$$
R^{2}=\frac{(3 / 2)(|\overrightarrow{\Delta \nu}|)^{2}}{\left(\epsilon^{*}\right)^{2}}=\frac{\left(\hat{\psi}_{1}\right)^{t} H_{1} \hat{\psi}_{1}}{\left(\hat{\psi}_{1}\right)^{t} \hat{\psi}_{1}} \text { for any } \hat{\psi}_{1} \neq\left(\begin{array}{l}
0 \\
0
\end{array}\right)\left(\text { i.e., }\left(\hat{\psi}_{1}\right)^{t} \hat{\psi}_{1}>0\right)
$$

In turn, with the aid of (i) Eq.(3.209), (ii) the fact that $H_{1}$ is a real symmetric matrix, and (iii) Rayleigh-Ritz Theorem [Ref.8, p.431], one concludes that, given any $\left(\alpha_{1}, \alpha_{2}, \alpha_{3}\right) \in D_{\alpha}$

$$
\sigma_{-}(\gamma) \leq R^{2} \leq \sigma_{+}(\gamma) \text { for any } \hat{\psi}_{1} \neq\left(\begin{array}{l}
0 \\
0
\end{array}\right)
$$

Because, Eqs.(3.199), (3.200) and (3.208) $\Rightarrow$

$$
\frac{1}{2}<\sigma_{-}(\gamma) \leq 1 \leq \sigma_{+}(\gamma) \text { and } R \geq 0 \text { for any } \hat{\psi}_{1} \neq\left(\begin{array}{l}
0 \\
0
\end{array}\right) \text { and any }\left(\alpha_{1}, \alpha_{2}, \alpha_{3}\right) \in D_{\alpha}
$$

Eq. $(3.210) \Leftrightarrow$

$$
\sqrt{\sigma_{-}(\gamma)} \leq R \leq \sqrt{\sigma_{+}(\gamma)} \text { for any } \hat{\psi}_{1} \neq\left(\begin{array}{l}
0 \\
0
\end{array}\right) \text { and any }\left(\alpha_{1}, \alpha_{2}, \alpha_{3}\right) \in D_{\alpha}
$$

Moreover, Eq.(3.211) and the Rayleigh-Ritz theorem also $\Leftrightarrow$ for any $\left(\alpha_{1}, \alpha_{2}, \alpha_{3}\right) \in D_{\alpha}$,

(i) $R=\sqrt{\sigma_{-}(\gamma)}$ for any $\hat{\psi}_{1}$ with $H_{1} \hat{\psi}_{1}=\sigma_{-}(\gamma) \hat{\psi}_{1}$ and $\hat{\psi}_{1} \neq\left(\begin{array}{l}0 \\ 0\end{array}\right)\left[\left(\alpha_{1}, \alpha_{2}, \alpha_{3}\right) \in D_{\alpha}\right]$

and

(ii) $R=\sqrt{\sigma_{+}(\gamma)}$ for any $\hat{\psi}_{1}$ with $H_{1} \hat{\psi}_{1}=\sigma_{+}(\gamma) \hat{\psi}_{1}$ and $\hat{\psi}_{1} \neq\left(\begin{array}{l}0 \\ 0\end{array}\right)\left[\left(\alpha_{1}, \alpha_{2}, \alpha_{3}\right) \in D_{\alpha}\right]$

To study the implication of Eqs.(3.212)-(3.214), first consider the special case in which $\alpha_{1}=\alpha_{2}=\alpha_{3}=\frac{\pi}{3}$, i.e., $\Delta P_{1} P_{2} P_{3}$ is equilateral. According to Eqs.(3.188) and (3.199), for this case, one has

$$
\gamma=\frac{9}{4}, \sigma_{-}\left(\frac{9}{4}\right)=\sigma_{+}\left(\frac{9}{4}\right)=1 \text { and } H_{1}=I_{2} \quad\left(\alpha_{1}=\alpha_{2}=\alpha_{3}=\frac{\pi}{3}\right)
$$

As such, for this special case, (i) Eq.(3.212) $\Rightarrow$

$$
R=1 \text { for any } \hat{\psi}_{1} \neq\left(\begin{array}{l}
0 \\
0
\end{array}\right)\left(\alpha_{1}=\alpha_{2}=\alpha_{3}=\frac{\pi}{3}\right)
$$

and (ii)

$$
H_{1} \hat{\psi}_{1}=I_{2} \hat{\psi}_{1}=\hat{\psi}_{1} \text { for any } \hat{\psi}_{1} \neq\left(\begin{array}{l}
0 \\
0
\end{array}\right)\left(\alpha_{1}=\alpha_{2}=\alpha_{3}=\frac{\pi}{3}\right)
$$

i.e., any $\hat{\psi}_{1} \neq\left(\begin{array}{l}0 \\ 0\end{array}\right)$ is an eigenvector of $H_{1}$ with unit eigenvalue if $\Delta P_{1} P_{2} P_{3}$ is equilateral. 
Next consider the case in which

$$
\left(\alpha_{1}, \alpha_{2}, \alpha_{3}\right) \neq\left(\frac{\pi}{3}, \frac{\pi}{3}, \frac{\pi}{3}\right) \text { and }\left(\alpha_{1}, \alpha_{2}, \alpha_{3}\right) \in D_{\alpha}
$$

According to Eqs.(3.185) and (3.188), Eq.(3.218) $\Leftrightarrow$

$$
0<\gamma<\frac{9}{4}
$$

Because (i) Eq.(3.200) implies that $\sigma_{-}(\gamma)<\sigma_{+}(\gamma)$ for each $\gamma$ with $0<\gamma<\frac{9}{4}$, (ii) two eigenvectors of $H_{1}$ associated with two distinct eigenvalues, respectively, must be linearly independent [Ref.8, p.268], and (iii) the $2 \times 2$ matrix $H_{1}$ can have at most two linearly independent eigenvectors, one concludes that, for each $\gamma$ with $0<\gamma<\frac{9}{4}$, the eigenspace of $H_{1}$ associated with either of the eigenvalues $\sigma_{-}(\gamma)$ and $\sigma_{+}(\gamma)$ is one-dimensional. As such, for a given $\gamma$ with $0<\gamma<\frac{9}{4}$, (i) $\hat{\psi}_{1}$, which is $2 \times 1$ nonzero real column matrix by definition, is an eigenvector of $H_{1}$ associated with the eigenvalue $\sigma_{-}(\gamma)$ if and only if

$$
\hat{\psi}_{1}=r_{-} \hat{\psi}_{1-}(\gamma), r_{-} \neq 0 \text { for any } \gamma \text { with } 0<\gamma<\frac{9}{4}
$$

where $r_{-}$is any real number $\neq 0$, and $\hat{\psi}_{1-}(\gamma)$ is the real eigenvector of $H_{1}$ introduced in Eq.(3.207), and (ii) $\hat{\psi}_{1}$ is the real eigenvector of $H_{1}$ associated with the eigenvalue $\sigma_{+}(\sigma)$ if and only if

$$
\hat{\psi}_{1}=r_{+} \hat{\psi}_{1+}(\gamma), r_{+} \neq 0 \text { for any } \gamma \text { with } 0<\gamma<\frac{9}{4}
$$

where $r_{+}$is any real number $\neq 0$, and $\hat{\psi}_{1+}(\gamma)$ is the real eigenvector of $H_{1}$ introduced in Eq.(3.207). In turn, by combining Eqs.(3.212)-(3.214) with the observations given in the above items (i) and (ii), one concludes that, for any $\gamma$ with $0<\gamma<\frac{9}{4}$, (ii)the lower bound $\sqrt{\sigma_{-}(\gamma)}$ is attained by $R$ if and only if $\hat{\psi}_{1}$ is in the form specified by Eqs.(3.220) and (ii) the upper bound of $\sqrt{\sigma_{+}(\gamma)}$ is attained by $R$ if and only if $\hat{\psi}_{1}$ is in the form specified by Eqs.(3.221). As such, for the case Eq.(3.218) (or equivalently the case Eq.(3.219)), Eq.(3.212) implies that $\sqrt{\sigma_{+}(\gamma)}$ and $\sqrt{\sigma_{-}(\gamma)}$, respectively, are the least upper bound and the greatest lower bound of $R$ for each $\gamma$ with $0<\gamma<\frac{9}{4}$.

As noted earlier, by its definition Eq.(3.208), $R$ is a measure of how large the numerical error norm associated with the gradient vector $\phi$ on the plane $\Gamma$ is amplified from that associated with the directional derivatives of $\phi$ evaluated along the three sides of $\Delta P_{1} P_{2} P_{3}$. On the other hand, for each given $\gamma$ with $0<\gamma<\frac{9}{4}$, Eq.(3.212) states that the value of $R$ can vary between the greatest lower bound $\sqrt{\sigma_{-}(\gamma)}$ and the least upper bound $\sqrt{\sigma_{+}(\gamma)}$. To minimize the possible value of the numerical error norm $|\overrightarrow{\Delta \nu}| / \sqrt{2}$ associated with the gradient vector of $\phi$ on $\Gamma$, against a given error norm $\epsilon^{*} / \sqrt{3}$ associated with the directional derivatives of $\phi$ evaluated along the three sides of $\Delta P_{1} P_{2} P_{3}$, it is imperative to minimize the least upper bound $\sqrt{\sigma_{+}(\gamma)}$.

According to the discussion given preceeding Eqs.(3.199)-(3.201), within its domain $0<\gamma<\frac{9}{4}$, the value of $\sigma_{+}(\gamma)$ increases monotonically from 1 to $+\infty$ as $\gamma$ decreases from $\frac{9}{4}$ to $0^{+}$. As such $\sqrt{\sigma_{+}(\gamma)}$ (i) reaches its minimal value 1 if and only of $\gamma=\frac{9}{4}$, and (ii) approaches $+\infty$ if and only if in the limit of $\gamma \rightarrow 0^{+}$.

Moreover, according to Eq.(3.186), $\gamma=\frac{9}{4}$ if and only if $\Delta P_{1} P_{2} P_{3}$ is equilateral. Thus $\sqrt{\sigma_{+}(\gamma)}$ reaches its minimal value 1 if and only if $\Delta P_{1} P_{2} P_{3}$ is equilateral, a result one would expect intutively. On the other hand, according to Eq.(3.187), $\gamma \rightarrow 0^{+}$and therefore $\sqrt{\sigma_{+}(\gamma)}$ approaches $+\infty$, if and only if the values of the two smaller internal angles of $\Delta P_{1} P_{2} P_{3}$ are both much less than one, as the case depicted in Fig.2(a). Luckily, even though $\Delta P_{1} P_{2} P_{3}$ depicted there must have a high aspect ratio, by no means the values of two of its internal angles must both be much less than one for a triangle with high aspect ratio. In fact, according to a discussion given in Sec.2, $\Delta P_{1} P_{2} P_{3}$ has a high aspect ratio if and only if the value of one of its internal angle is much less than one. As such, a triangle with the value of only one of its internal angle being much less than one, such as the case depicted in Fig.2(b), is also associated with a high aspect ratio, In fact, according to Eq.(3.202), $\gamma=2$ for any right triangle even if the value of its smallest angle is much less than one and thus it has a high aspect ratio. Moreover, according to Eq.(3.203), for this case,

$$
\text { the least bound of } \mathrm{R}=\sqrt{\sigma_{+}(2)}=\sqrt{\frac{3}{2}} \approx 1.22 \text { ( for a right triangle). }
$$


In other words, the least upper bound of $R$ can be quiet close to its minimal value 1 even if the right triangle $\Delta P_{1} P_{2} P_{3}$ has a high aspect ratio. Thus, as long as one of its internal angles is close to the right angle, the least upper bound of the error amplification factor $R$ associated with $\Delta P_{1} P_{2} P_{3}$ is quiet close to the minimal value 1 even if the triangle has a large aspect ratio.

From the above theoretical discussions, one concludes that the accuracy of gradient calculation of a solution variable over a triangular spatial grid is tied to the shape factor $\gamma$ of individual triangles from which the grid is built. Specifically, if the values of $\gamma$ associated with the component triangles are close to its maximal value $\frac{9}{4}$ (which is exactly attained if and only if the triangle is equilateral), the upper bounds of the error amplification factors $R$ associated with the component triangles will be relatively close to its minimal value 1. It is also shown that the value of $\gamma$ associated with a triangle with a large aspect ratio can be close to the ideal maximal value $\frac{9}{4}$ as long as one of its internal angles is close to the right triangle (i.e., a 90 degree angle). As such, accuracy deterioration of gradient calculation due to the high aspect ratio associated with the triangular grid used can be avoided if the grid is built from triangles with each of them possessing the property that one of its internal angles is close to the right angle. As will be shown in the following sections, the above theoretical predictions have been confirmed numerically.

\section{Results and Discussion}

Numerical algorithms to circumvent numerical instability and accuracy issues for high aspect ratio triangular/tetrahedral meshes are being implemented in the software framework. The in-house CFD code framework $e z 4 d^{3}$ based on various schemes that belong to the CESE family has been continuously under development since 2004. This software framework has been written using a combined object-oriented and generic programming paradigms in the $\mathrm{C}++$ programming language. Light-weight object-oriented hierarchy is used in conjunction with heavy use of template classes and functions to allow compile time polymorphism. Different conservation laws can be plugged in with templates that represent physics. Currently, the software supports either triangular/tetrahedral or quadrilateral/hexahedral unstructured meshes. Both multi-thread (based on low-level POSIX thread) and message passing interface (MPI) paradigms are used to facilitate large-scale parallel computations. Each MPI process within a computational node can be executed in multithread mode to further enhance parallel performance, especially for a memory bound multi-domain layout. A communication map is used for data transfer among interface zones. For a large mesh, of the order of a billion elements, each unstructured block can be built with its own connectivity and nodes. A global communication map is then used to join all the independent blocks in the parallel computations. This arrangement allows the grid generation process to always have a low memory requirement. The interfaces among blocks can be continuous or discontinuous. A continuous interface mesh ensures better solution accuracy for unsteady flow computations. Both second- and fourth-order CESE numerical schemes are implemented for general conservation laws including Euler and Navier-Stokes equations in the software framework. The time accurate local time-stepping (TALTS) scheme, ${ }^{3-6}$ that allows all the elements in a mesh to march at an approximately uniform CFL number without violating flux conservation in time, is used to enhance parallel performance (smaller size elements do more computations than the larger ones) and numerical accuracy.

High aspect ratio meshes are used routinely in viscous flow computations. In this section, several practical test examples are discussed to demonstrate the effectiveness of the CESE method to deal with high-aspect ratio meshes inside the boundary layer without any degradation in accuracy or numerical stability. The gradient evaluation procedures that enables CESE to use high-aspect ratio meshes are detailed in a previous paper. ${ }^{4}$ Furthermore, all the results featured here were computed with the use of the TALTS scheme, implemented in $e z 4 d$, to maintain computational efficiency and accuracy at the same time.

\section{IV.A. Acoustic Wave Propagation along High-Aspect Ratio Boundary-Layer Mesh}

The TALTS method is designed to handle large grid size disparities in a discretized domain. One frequently encountered problem in flow-physics is to simulate acoustic waves propagating through a viscous mesh. Computing acoustic waves using such a non-uniform mesh is quite challenging. Figure (6) shows a rectangular domain with a clustered triangular mesh with an aspect ratio $(\eta)$ around 225 near the bottom boundary. A constant time step computation results in significant phase error due to the large CFL number disparities. The wave amplitude is also incorrectly damped. In contrast, the TALTS computation maintains a rather 


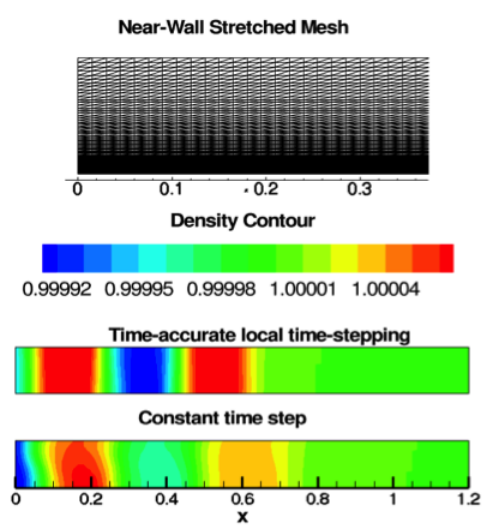

Figure 6. Acoustic waves propagating through a domain with clustered mesh, showing density contours at a time instant.

uniform CFL number (around 0.8) and thus results in much improved accuracy in both amplitude and phase throughout the domain. The results indicate that high aspect ratio triangular mesh poses no numerical issues for acoustic wave propagation. Capability to resolve acoustic or other physical waves inside the boundary layer is essential for direct numerical simulations of transitional or turbulent boundary layers.

\section{IV.B. Mach 3 Isothermal Laminar Boundary Layer}

Surface heating prediction has been one of the most important topics in many supersonic or hypersonic computational investigations. Accurately predicting the surface heat flux in a highly cooled boundary layer has important implications in vehicle thermal shield and aerodynamic design. Of interest is the isothermal boundary layer development behind the leading-edge bow shock of a blunt body. To isolate the boundary layer from shocks for numerical accuracy studies, a Mach 3 flow (with a free-stream temperature of 200 $\mathrm{K}$ ) over a highly cooled boundary layer with $T_{\text {wall }} / T_{\text {wall,adiabatic }}=0.2$ is numerically computed. Due to the presence of the boundary layer, there is a weak leading edge shock that would make the free-stream conditions of the boundary layer deviate slightly from the Mach 3 conditions. The computational domain is a $1.2 \mathrm{~m} \times 0.1 \mathrm{~m}$ rectangle (the height is about 5 boundary-layer thicknesses at the exit). A structured quadrilateral mesh with different sizes is sliced to form triangular elements for computations. The computed velocity and temperature profiles (with a mesh of 128,000 triangular elements and a maximum aspect ratio $(\eta)$ of about 1500) at the location of $R e=10^{5}$ are compared with compressible similarity boundary layer solutions in Fig.7, along with the surface heat flux distribution along the streamwise direction. The results show good agreement for laminar surface heat flux predictions.

\section{IV.C. RANS Computations for a Mach 2 Adiabatic Boundary Layer}

Meshes with high aspect ratio elements are most frequently used for Reynolds Averaged Navier-Stokes (RANS) computations. To resolve the viscous sublayer, log layer, and all the way up to the boundary layer edge, the near wall $\mathrm{y}^{+}$value has to be kept around or smaller than one. This step size is several orders of magnitude smaller than that along the strewamwise direction, which implies a very large aspect ratio. RANS computations are carried out using both Sparlart-Allmaras and Mentor's SST models. ${ }^{10}$ Both skin friction coefficients and turbulent mean streamwise profiles are compared with results from the widely used NASA CFL3D code. A triangular mesh with about 105,000 elements and an aspect ratio $(\eta)$ of around 3000 obtained by slicing the quad mesh downloaded from the turbulent resource website, ${ }^{10}$ was used for a Mach 2 adiabatic boundary layer. The agreement with structured mesh solutions is quite good (see Fig.8). It indicates the numerical dissipation treatment for the high aspect ratio unstructured meshes is adequate in resolving RANS equations.

\section{IV.D. Mach 11 Double Cone Benchmark Problem}

The CUBRC wind tunnel tests ${ }^{11}$ for hypersonic flows over a $15^{\circ}-25^{\circ}$ double cone is often used to validate surface heating and the capability of CFD codes in predicting laminar or turbulent shock boundary 


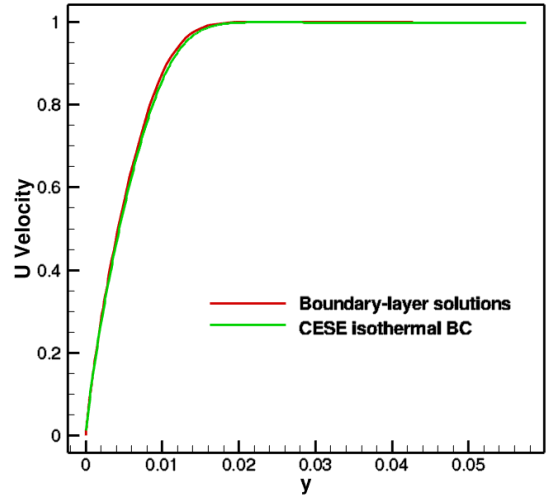

(a)

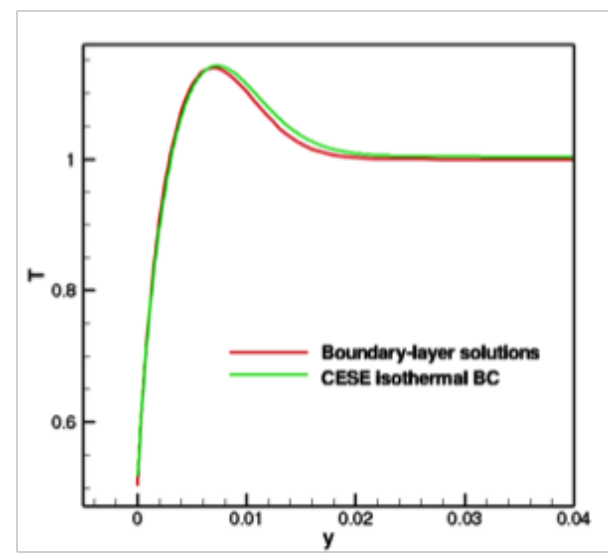

(b)

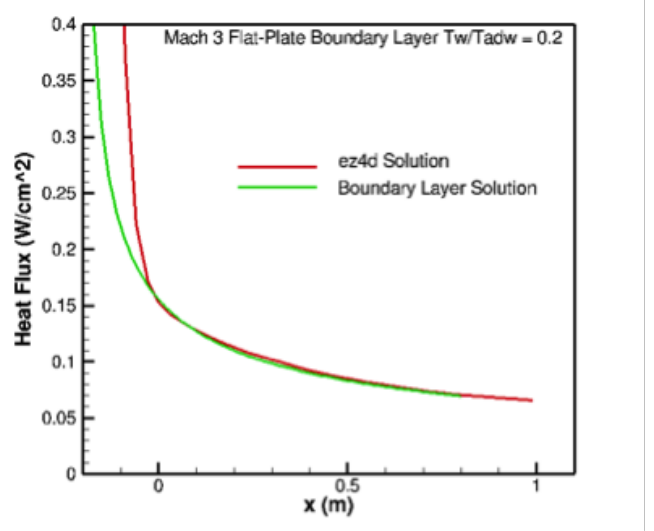

(c)

Figure 7. Comparison of Navier-Stokes solutions with compressible boundary-layer solutions at Re $=10^{5}$ for a Mach 3 flate-plate flow with $T_{\text {wall }} / T_{\text {wall, adiabatic }}=0.2:$ (a) streamwise velocity (non-dimensionalized by $u_{\infty}$ ) (b) temperature (non-dimensionalized by $T_{\infty}$ ) and (c) heat flux distribution along the streamwise direction.

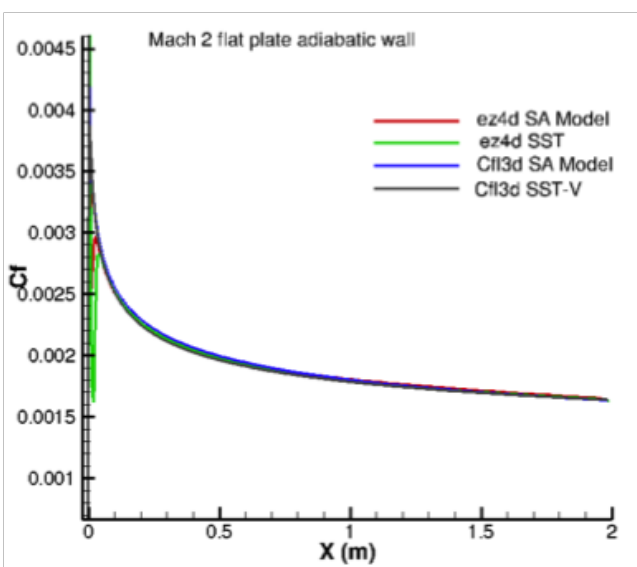

(a)

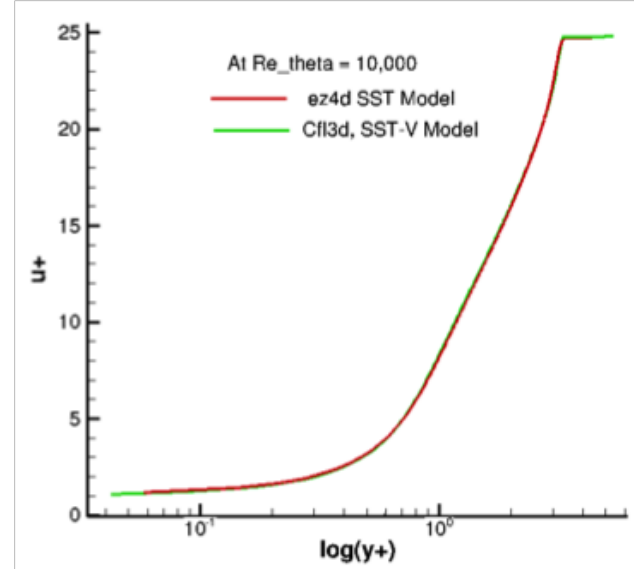

(b)

Figure 8. Comparison of RANS solutions with results from CFL3D for a Mach 2 flat-plate turbulent boundary layer flow with adiabatic walls: (a) skin friction coefficient (b) turbulent velocity profiles at $R e_{\theta}=10,000$. 
layer interaction. Laminar calculations for the Run 35 case in Ref.[11] are carried out and the computed Mach number contours are shown in Fig.9 below. In all of the plots shown, the lengths have been nondimensionalized with the length $L$ of the first section of the cone. A triangular mesh with about 313,000 elements and an aspect ratio $(\eta)$ of about 400 near the wall was used for the computations. The separation bubble around the corner and the big subsonic bubble around the second ramp are clearly captured. The cone surface pressure coefficient and Stanton number are compared with the experimental data in Fig.10. The agreement of $C_{p}$ is quite good, while the current results under-predict the surface heat flux. A grid sensitivity study is currently underway to sort out the heat flux discrepancies.

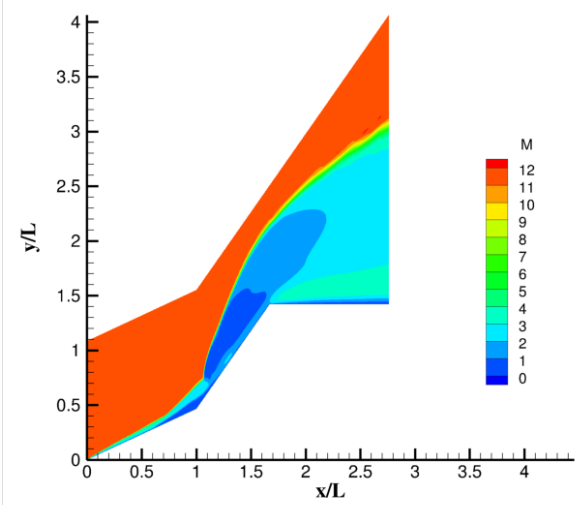

Figure 9. Computed Mach number contours for the Mach 11.3 flow over the CUBRC 15-25 double cone.

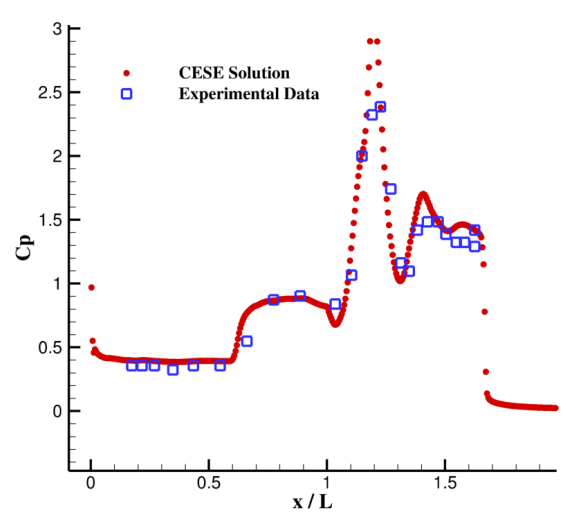

(a)

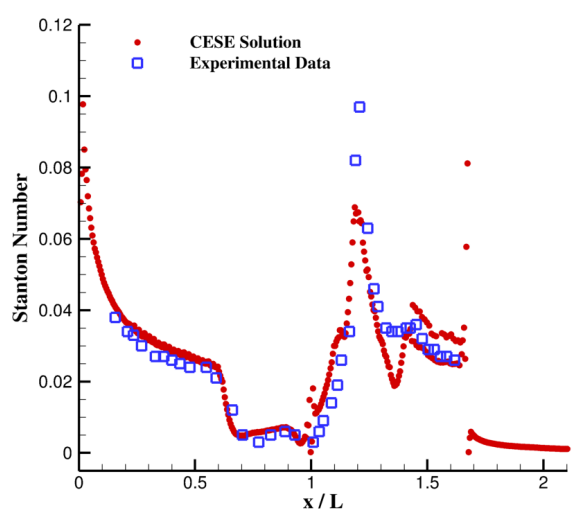

(b)

Figure 10. Comparison of computed surface pressure coffeicent and Stanton number with experimental data for the Mach 11.3 flow over a double cone: (a) $C_{p}$ (b) Stanton number.

\section{Conclusion}

This work discusses the fundamental accuracy issues when a mesh with very large aspect ratio elements is used in CFD simulations, especially inside the viscous boundary layer. Sources of inaccuracy as an outcome of triangular shapes are identified theoretically, followed by a discussion on potential remedies. The current status about the application of the CESE method in steady or unsteady computations for acoustic waves or flow over a viscous boundary layer with large aspect ratio triangular meshes is briefly discussed, to demonstrate the effectiveness of the gradient evaluation procedures to deal with mesh-related geometrical difficulties. 


\section{Appendix A}

Proposition 1 presented in Section II will be proved here. To prove Eqs.(2.20) and (2.21), first note that Eq.(2.17) implies that each $\left(\alpha_{k 1}, \alpha_{k 2}, \alpha_{k 3}\right)$ must meet one of the following two mutually exclusive conditions:

$$
\text { (a) } \frac{\pi}{2}>\max \left\{\alpha_{k 1}, \alpha_{k 2}, \alpha_{k 3}\right\}>0 \text { with }\left\{k_{1}, k_{2}, k_{3}\right\}=\{1,2,3\}
$$

and

$$
\text { (b) } \pi>\max \left\{\alpha_{k 1}, \alpha_{k 2}, \alpha_{k 3}\right\} \geq \frac{\pi}{2} \text { with }\left\{k_{1}, k_{2}, k_{3}\right\}=\{1,2,3\}
$$

Because, in the interval $0 \leq \alpha \leq \pi / 2, \sin \alpha$ increases monotonically from 0 to 1 as $\alpha$ increases from 0 to $\pi / 2$, it is seen that, for the case Eq.(A.1), Eq.(2.20) is a direct result of Eqs.(A.1) and (2.15). In the following, for the case Eq.(A.2), we will prove the Eq.(2.21) is also a result of Eqs.(2.15) and (2.17).

First we will prove that, for the case Eq.(A.2),

$$
\alpha_{k 2} \neq \max \left\{\alpha_{k 1}, \alpha_{k 2}, \alpha_{k 3}\right\} \text { and } \alpha_{k 3} \neq \max \left\{\alpha_{k 1}, \alpha_{k 2}, \alpha_{k 3}\right\} \quad \text { ( case Eq.(A.2)) }
$$

To prove the first part of Eq.(A.3) by contradiction, assume

$$
\pi>\alpha_{k 2}=\max \left\{\alpha_{k 1}, \alpha_{k 2}, \alpha_{k 3}\right\} \geq \frac{\pi}{2} \text { and case Eq.(A.2) }
$$

and let

$$
\overline{\alpha_{k 2}} \stackrel{\text { def }}{=} \pi-\alpha_{k 2}
$$

Because Eq.(A.5) $\Leftrightarrow \alpha_{k 2}=\pi-\overline{\alpha_{k 2}}$, Eq.(A.4) and the second part of Eq.(2.17), respectively, imply that

$$
\frac{\pi}{2} \geq \overline{\alpha_{k 2}}>0 \quad \text { for case Eq.(A.4) }
$$

and

$$
\overline{\alpha_{k 2}}=\alpha_{k 1}+\alpha_{k 3}
$$

In turn, Eqs.(A.6) and (A.7) $\Rightarrow$

$$
\frac{\pi}{2} \geq \alpha_{k 1}+\alpha_{k 3}>0 \quad \text { for case Eq.(A.4) }
$$

On the other hand, according to the first part of Eq.(2.17), $\alpha_{k 1}>0$ and $\alpha_{k 3}>0$. Thus, Eq.(A.8) implies that

$$
\frac{\pi}{2}>\alpha_{k 1}>0 \text { and } \frac{\pi}{2}>\alpha_{k 3}>0 \quad \text { for case Eq.(A.4) }
$$

Next, because $\sin (\pi-\alpha)=\sin \alpha$ for any real number $\alpha$, Eq.(A.5) implies that

$$
\sin \alpha_{k 2}=\sin \overline{\alpha_{k 2}}
$$

In turn, with the aid of Eq.(A.10), Eq.(2.15) implies that

$$
1 \geq \sin \alpha_{k 1} \geq \sin \overline{\alpha_{k 2}} \geq \sin \alpha_{k 3}>0
$$

On the other hand, because, in the interval $0 \leq \alpha \leq \pi / 2, \sin \alpha$ increases monotonically from 0 to 1 as $\alpha$ increases from 0 to $\pi / 2$, for the current case Eqs.(A.6) and (A.9), Eq.(A.11) $\Leftrightarrow$

$$
\frac{\pi}{2} \geq \alpha_{k 1} \geq \overline{\alpha_{k 2}} \geq \alpha_{k 3}>0 \quad \text { (case Eqs.(A.6) and (A.9)) }
$$

By substituting Eq.(A.7) into a result of Eq.(A.12), we have

$$
\alpha_{k 1} \geq \overline{\alpha_{k 2}}=\alpha_{k 1}+\alpha_{k 3}>0 \quad \text { (case Eqs.(A.6) and (A.9)) }
$$

which leads to the result $0 \geq \alpha_{k 3}$ and thus a contradiction to the first part of Eq.(2.17). As such the assumption Eq.(A.4) is false and therefore the proposition given in the first part of Eq.(A.3) is required for consistency to Eqs.(2.15) and (2.17). 
Because of Eq.(A.3), for the case Eq.(A.2), consistency to Eqs.(2.15) and (2.17) requires that

$$
\pi>\alpha_{k 1}=\max \left\{\alpha_{k 1}, \alpha_{k 2}, \alpha_{k 3}\right\} \geq \frac{\pi}{2} \text { for case Eq.(A.2) }
$$

Because,

$$
\pi>\alpha_{k 1} \geq \frac{\pi}{2} \Leftrightarrow \frac{\pi}{2} \geq \overline{\alpha_{k 1}} \stackrel{\text { def }}{=} \pi-\alpha_{k 1}>0
$$

one can show that, for the case Eq.(A.4), Eq.(2.17) $\Leftrightarrow$

$$
\left.\begin{array}{l}
\frac{\pi}{2} \geq \overline{\alpha_{k 1}}=\pi-\alpha_{k 1}=\alpha_{k 2}+\alpha_{k 3}>\alpha_{k 2}>0 \\
\text { and } \\
\frac{\pi}{2} \geq \overline{\alpha_{k 1}}=\pi-\alpha_{k 1}=\alpha_{k 2}+\alpha_{k 3}>\alpha_{k 3}>0
\end{array}\right\} \quad \text { for case Eq.(A.14) }
$$

To search for the extra conditions on $\alpha_{k 1}, \alpha_{k 2}$ and $\alpha_{k 3}$ required for consistency to Eq.(2.15), note that, by using the definition of $\overline{\alpha_{k 1}}$ given above, and the relation $\sin (\pi-\alpha)=\sin \alpha$ for any real number $\alpha$, it is seen that Eq. $(2.15) \Leftrightarrow$

$$
1 \geq \sin \overline{\alpha_{k 1}} \geq \sin \alpha_{k 2} \geq \sin \alpha_{k 3}>0 \quad(\Leftrightarrow \text { Eq. (2.15)) }
$$

On the other hand, because (i) in the interval $0 \leq \alpha \leq \frac{\pi}{2}, \sin \alpha$ increases monotonically from 0 to 1 as $\alpha$ increases from 0 to $\frac{\pi}{2}$, and (ii) Eq.(A.16) $\Rightarrow \frac{\pi}{2} \geq \overline{\alpha_{k 1}}>0, \frac{\pi}{2}>\alpha_{k 2}>0$ and $\frac{\pi}{2}>\alpha_{k 3}>0$, for the case Eq.(A.16), Eq.(A.17) $\Leftrightarrow$

$$
\frac{\pi}{2} \geq \overline{\alpha_{k 1}}=\pi-\alpha_{k 1}>\alpha_{k 2} \geq \alpha_{k 3}>0 \quad \text { case Eq.(A.16) }
$$

As such, for the case Eq.(A.14), Eqs.(A.16) and (A.18) $\Leftrightarrow$ Eqs.(2.15) and (2.17). On the other hand, it can be shown that Eqs.(A.16) and (A.18) $\Leftrightarrow$

$$
\frac{\pi}{2} \geq \pi-\alpha_{k 1}=\alpha_{k 2}+\alpha_{k 3}>\alpha_{k 2} \geq \alpha_{k 3}>0 \quad \text { case Eq.(A.14) }
$$

As such, for the case Eq.(A.14), it has been shown that Eqs.(2.15) and (2.17) $\Leftrightarrow$ Eq.(A.19). Because, it was shown earlier that, the case Eq.(A.14) is the only subcase of Eq.(A.2) which can be consistent with Eqs.(2.15) and (2.17), one concludes that, for the case Eq.(A.2), Eqs.(2.15) and (2.17) $\Leftrightarrow$ Eq.(A.19). Because, Eqs.(A.19) $\Leftrightarrow$ (i) Eq.(2.21) and (ii) the second part of Eq.(2.17), it has been proved that, for the case Eq.(A.2), Eq.(2.21) is a result of Eqs.(2.15) and (2.17).

It was shown earlier that, (i) as a result of Eq.(2.17), each $\left(\alpha_{k 1}, \alpha_{k 2}, \alpha_{k 3}\right)$ must meet one of the two mutually exclusive conditions Eqs.(A.1) and (A.2); and (ii) for the case Eq.(A.1), Eq.(2.20) is a result of Eq.(2.15). By combining the results with the result presented in the last paragraph, one concludes that Eq.(2.20) and (2.21) imply either Eq.(2.20) or Eq.(2.21). In the following, Eqs.(2.22)-(2.25) will be proved using Eqs.(2.16) and (2.19)-(2.21).

Note that: (i) Eqs. $(2.17)$ and $(2.20) \Leftrightarrow$

$$
\frac{\pi}{2}>\alpha_{k 1} \geq \alpha_{k 2}>0 \text { and } \frac{\pi}{3} \geq \alpha_{k 3}>0 \quad \text { case Eqs.(2.17) and (2.20) }
$$

and (ii) Eqs.(2.17) and (2.21) $\Leftrightarrow$

$$
\frac{\pi}{2}>\geq \pi-\alpha_{k 1}>\alpha_{k 2}>0 \text { and } \frac{\pi}{4}>\alpha_{k 3}>0 \quad \text { case Eqs.(2.17) and (2.21) }
$$

Because (i) in the interval $0 \leq \alpha<\frac{\pi}{2}$, $\sin \alpha$ increases monotonically from 0 to 1 as $\alpha$ increases from 0 to $\frac{\pi}{2}$; and (ii) in the interval $0<\alpha \leq \frac{\pi}{2}, 0<\sin \alpha \leq 1$; and (iii) $\sin (\pi-\alpha)=\sin \alpha$ for any real number $\alpha$, one concludes that (i) Eq.(A.20) $\Rightarrow$

$$
\frac{\sin \alpha_{k 1}}{\sin \alpha_{k 2}} \geq 1 \text { and } \sin \left(\frac{\pi}{3}\right)=\frac{\sqrt{3}}{2} \geq \sin \alpha_{k 3}>0 \quad \text { (case Eq.(A.20)) }
$$

and (ii) Eq.(A.21) $\Rightarrow$

$$
\frac{\sin \alpha_{k 1}}{\sin \alpha_{k 2}}=\frac{\sin \left(\pi-\alpha_{k 1}\right)}{\sin \alpha_{k 2}}>1 \text { and } \sin \left(\frac{\pi}{4}\right)=\frac{1}{\sqrt{2}}>\sin \alpha_{k 3}>0 \quad \text { (case Eq.(A.21)) }
$$


As such, for the case Eq.(A.20), Eqs.(2.16) and (A.22) $\Rightarrow$ (i)

$$
\eta \geq \frac{1}{\sin \alpha_{k 3}} \geq \frac{2}{\sqrt{3}} \approx 1.155 \quad \text { (case Eq.(A.20)) }
$$

and (ii)

$$
\begin{gathered}
\eta=\frac{2}{\sqrt{3}} \text { if and only if } \alpha_{k 1}=\alpha_{k 2}=\alpha_{k 3}=\frac{\pi}{3} \\
\text { i.e., } \alpha_{1}=\alpha_{2}=\alpha_{3}=\frac{\pi}{3} \quad \text { (case Eq.(A.20)) }
\end{gathered}
$$

On the other hand, for the case Eq.(A.21), Eqs.(2.16) and (A.23) $\Rightarrow$

$$
\eta>\frac{1}{\sin \alpha_{k 3}}>\sqrt{2} \approx 1.414>\frac{2}{\sqrt{3}} \quad(\text { case Eq.(A.21) }
$$

Eqs.(2.22) and (2.23) now follow directly from Eqs.(A.20)-(A.26).

To prove Eqs.(2.24) and (2.25) note that both Eqs.(2.20) and (2.21) $\Rightarrow$

$$
\frac{\pi}{2}>\alpha_{k 2} \geq \alpha_{k 3}>0 \quad \text { for case Eqs.(2.20) or case Eq.(2.21) }
$$

By using the fact that, in the interval $0<\alpha \leq \frac{\pi}{2}, \cot \alpha$ increases monotonically from 0 to $+\infty$ as $\alpha$ decreases from $\frac{\pi}{2}$ to 0 , (i) Eq.(A.27) $\Rightarrow$

$$
\cot \alpha_{k 3} \geq \cot \alpha_{k 2}>0 \quad \text { for case Eqs.(2.20) or case Eq.(2.21) }
$$

and (ii)

$$
\lim _{\alpha \rightarrow 0^{+}} \cot \alpha=+\infty
$$

On the other hand, by combining Eqs.(2.19) and (A.28), one has

$$
2 \cot \alpha_{k 3} \geq \eta>\cot \alpha_{k 3}>0 \quad \text { for case Eqs.(2.20) or case Eq.(2.21) }
$$

In turn, with the aid of Eqs.(A.27) and (A.29), Eq.(A.30) $\Rightarrow$ both Eqs.(2.24) and (2.25). QED.

\section{Appendix B}

In this appendix, Eqs.(3.185)-(3.187) will be proved using Eq.(3.139).

To proceed, note that, with the aid of Eq.(3.50), Eq.(3.139) $\Rightarrow$

$$
\begin{aligned}
& \gamma\left(\alpha_{1}, \alpha_{2}, \alpha_{3}\right)=\gamma\left(\alpha_{1}, \alpha_{2}, \pi-\alpha_{1}-\alpha_{2}\right) \\
& =g\left(\alpha_{1}, \alpha_{2}\right) \stackrel{\text { def }}{=} \sin ^{2} \alpha_{1}+\sin ^{2} \alpha_{2}+\sin ^{2}\left(\alpha_{1}+\alpha_{2}\right),\left(\alpha_{1}, \alpha_{2}, \alpha_{3}\right) \in D_{\alpha}
\end{aligned}
$$

where the domain of the function $g$ is

$$
D(g) \stackrel{\text { def }}{=}\left\{\left(\alpha_{1}, \alpha_{2}\right) \mid \alpha_{1}>0, \alpha_{2}>0 \text { and } \alpha_{1}+\alpha_{2}<\pi\right\}
$$

Given Eqs.(B.1) and (B.2), next we will prove Proposition B-1:

Proposition B-1: Within its domain $D(g)$, the global maximum of the function $g$ is $\frac{9}{4}$, and it is attained at and only at

$$
\left(\alpha_{1}, \alpha_{2}\right)=(\pi / 3, \pi / 3)
$$

Proof: By using Eq.(B.1) and some trigonometric identities, one has

$$
\begin{gathered}
\frac{\partial g}{\partial \alpha_{1}}=\sin \left(2 \alpha_{1}\right)+\sin \left[2\left(\alpha_{1}+\alpha_{2}\right)\right]=2\left[\sin \left(2 \alpha_{1}+\alpha_{2}\right)\right]\left(\cos \alpha_{2}\right) \\
\frac{\partial g}{\partial \alpha_{2}}=\sin \left(2 \alpha_{2}\right)+\sin \left[2\left(\alpha_{1}+\alpha_{2}\right)\right]=2\left[\sin \left(\alpha_{1}+2 \alpha_{2}\right)\right]\left(\cos \alpha_{1}\right)
\end{gathered}
$$




$$
\begin{aligned}
& \frac{\partial^{2} g}{\partial \alpha_{1}^{2}}=4 \cos \left(2 \alpha_{1}+\alpha_{2}\right)\left(\cos \alpha_{2}\right) \\
& \frac{\partial^{2} g}{\partial \alpha_{2}^{2}}=4 \cos \left(\alpha_{1}+2 \alpha_{2}\right)\left(\cos \alpha_{1}\right)
\end{aligned}
$$

and

$$
\frac{\partial^{2} g}{\partial \alpha_{1} \partial \alpha_{2}}=2 \cos \left[2\left(\alpha_{1}+\alpha_{2}\right)\right]
$$

By using the last expression in each of Eq.(B.4) and (B.5), it is seen that, within $D(g)$, the extremum condition [Ref.12, p. 304]

$$
\frac{\partial g}{\partial \alpha_{1}}=\frac{\partial g}{\partial \alpha_{2}}=0, \quad\left(\alpha_{1}, \alpha_{2}\right) \in D(g)
$$

$\Leftrightarrow$ one of the following four possible cases:

(a) $\cos \alpha_{1}=\cos \alpha_{2}=0$; (b) $\cos \alpha_{2}=0$ and $\sin \left(\alpha_{1}+2 \alpha_{2}\right)=0$; (c) $\cos \alpha_{1}=0$ and $\sin \left(2 \alpha_{1}+\alpha_{2}\right)=0$; and (d) $\sin \left(2 \alpha_{1}+\alpha_{2}\right)=\sin \left(\alpha_{1}+2 \alpha_{2}\right)=0$

In $D(g), 0<\alpha_{1}, \alpha_{2}<\pi$. Thus case (a) $\Leftrightarrow \alpha_{1}=\alpha_{2}=\pi / 2 \Rightarrow \alpha_{1}+\alpha_{2}=\pi$. On the other hand, according to Eq.(B.2), $\alpha_{1}+\alpha_{2}=\pi \Rightarrow\left(\alpha_{1}, \alpha_{2}\right) \notin D(g)$. Thus case (a) cannot occur for any $\left(\alpha_{1}, \alpha_{2}\right) \in D(g)$.

For any $\left(\alpha_{1}, \alpha_{2}\right) \in D(g), \cos \alpha_{2}=0 \Leftrightarrow \alpha_{2}=\pi / 2$. On the other hand, with $\alpha_{2}=\frac{\pi}{2}, \sin \left(\alpha_{1}+2 \alpha_{2}\right)=$ $\sin \left(\pi+\alpha_{1}\right)=-\sin \alpha_{1}$. Because $\sin \alpha_{1}>0$ for any $\left(\alpha_{1}, \alpha_{2}\right) \in D(g)$, case (b) also cannot occur for any $\left(\alpha_{1}, \alpha_{2}\right) \in D(g)$. Similarly one can show that case (c) also cannot occur for any $\left(\alpha_{1}, \alpha_{2}\right) \in D(g)$.

Because $2 \alpha_{1}+\alpha_{2}=\left(\alpha_{1}+\alpha_{2}\right)+\alpha_{1}$ and $\alpha_{1}+2 \alpha_{2}=\left(\alpha_{1}+\alpha_{2}\right)+\alpha_{2}$, it is seen that, for any $\left(\alpha_{1}, \alpha_{2}\right) \in$ $D(g), 0<2 \alpha_{1}+\alpha_{2}<2 \pi$ and $0<\alpha_{1}+2 \alpha_{2}<2 \pi$. Thus case $(\mathrm{d}) \Leftrightarrow$

$$
2 \alpha_{1}+\alpha_{2}=\alpha_{1}+2 \alpha_{2}=\pi
$$

for any $\left(\alpha_{1}, \alpha_{2}\right) \in D(g)$. Because Eq.(B.10) $\Leftrightarrow$ Eq.(B.3), one concludes that, within $D(g)$, the extremum condition Eq.(B.9) is met if and only if $\alpha_{1}=\alpha_{2}=\frac{\pi}{3}$.

Let $\alpha_{1}=\alpha_{2}=\frac{\pi}{3}$. Then, Eqs.(B.5)-(B.7) $\Rightarrow$

$$
\frac{\partial^{2} g}{\partial \alpha_{1}^{2}}=\frac{\partial^{2} g}{\partial \alpha_{2}^{2}}=-2 \text { and } \frac{\partial^{2} g}{\partial \alpha_{1} \partial \alpha_{2}}=-1 \quad\left(\alpha_{1}=\alpha_{2}=\pi / 3\right)
$$

In turn, Eq.(B.11) $\Rightarrow$

$$
\frac{\partial^{2} g}{\partial \alpha_{1}^{2}} \frac{\partial^{2} g}{\partial \alpha_{2}^{2}}-\left(\frac{\partial^{2} g}{\partial \alpha_{1} \partial \alpha_{2}}\right)^{2}=3>0 \quad\left(\alpha_{1}=\alpha_{2}=\pi / 3\right)
$$

and

$$
\frac{\partial^{2} g}{\partial \alpha_{1}^{2}}=-2<0 \quad\left(\alpha_{1}=\alpha_{2}=\pi / 3\right)
$$

when $\alpha_{1}=\alpha_{2}=\pi / 3$. Because (i) within $D(g)$, the extremum condition, Eq.(B.9) is met if and only if $\alpha_{1}=\alpha_{2}=\pi / 3$; (ii) Eqs.(B.12) and (B.13) $\Rightarrow$ a relative maximum of $g$ occurs when $\alpha_{1}=\alpha_{2}=\pi / 3$ [Ref.12, p. 312]; (iii) $D(g)$ is an open domain [Ref.13, p.28]; and (iv)

$$
g(\pi / 3, \pi / 3)=\frac{9}{4}
$$

one concludes that the global maximum of $g$ in $D(g)$ is $9 / 4$ and it is attained at and only at $\left(\alpha_{1}, \alpha_{2}\right)=$ $(\pi / 3, \pi / 3)$. QED.

With the aid of Proposition B-1. Eqs.(B.1) and (B.2) now imply (i) Eq.(3.186), (ii)

$$
0<g\left(\alpha_{1}, \alpha_{2}\right)<9 / 4 \text { if }\left(\alpha_{1}, \alpha_{2}\right) \in D(g) \text { and }\left(\alpha_{1}, \alpha_{2}\right) \neq(\pi / 3, \pi / 3)
$$

and (iii)

$$
\lim _{\left(\alpha_{1}, \alpha_{2}\right) \rightarrow\left(0^{+}, 0^{+}\right)} g\left(\alpha_{1}, \alpha_{2}\right)=\lim _{\left(\alpha_{1}, \alpha_{2}\right) \rightarrow\left(0^{+}, \pi^{-}\right)} g\left(\alpha_{1}, \alpha_{2}\right)=\lim _{\left(\alpha_{1}, \alpha_{2}\right) \rightarrow\left(\pi^{-}, 0^{+}\right)} g\left(\alpha_{1}, \alpha_{2}\right)=0
$$

Moreover, because (i) $g$ is continuous over $D(g)$, and (ii) points $(0,0),(0, \pi)$ and $(\pi, 0)$ on the $\alpha_{1}-\alpha_{2}$ plane (see Fig.11) are limit points [Ref.13, p.28] of $D(g)$ one concludes from Eqs.(B.14)-(B.16) that, given 


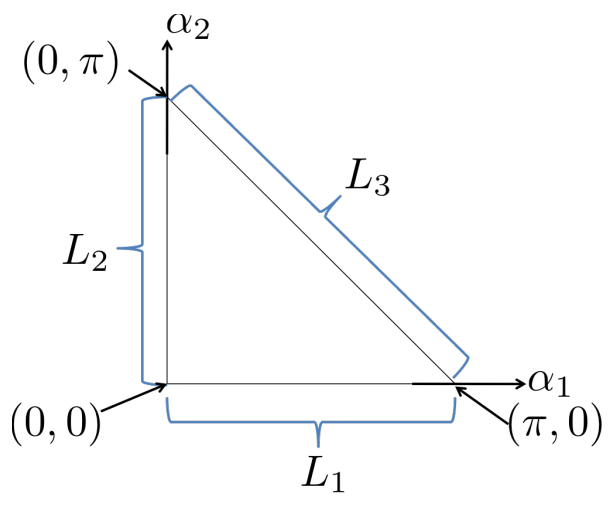

Figure 11. Representation of a triangular element in $\alpha_{1}-\alpha_{2}$ space.

any number $x$ with $0<x \leq 9 / 4$, there exists an $\left(\alpha_{2}, \alpha_{2}\right) \in D(g)$ such that $g\left(\alpha_{2}, \alpha_{2}\right)=x$. Thus, the range of $g$ over $D(g)$ is $0<g \leq 9 / 4$. In turn, with the aid of Eqs.(B.1) and (B.2), one concludes that the range of $\gamma$ over $D_{\alpha}$ is defined by Eq.(3.185).

To prove Eq.(3.187), first note that continuity of $g$ over $D(g)$ coupled with Eqs.(B.14) and (B.15) implies that, for any given fixed point $\left(\alpha_{1}^{0}, \alpha_{2}^{0}\right) \in D(g)$, (i) $g\left(\alpha_{1}^{0}, \alpha_{2}^{0}\right)$ is a fixed number $>0$, and (ii)

$$
\lim _{\left(\alpha_{2}, \alpha_{2}\right) \rightarrow\left(\alpha_{1}^{0}, \alpha_{2}^{0}\right)} g\left(\alpha_{1}, \alpha_{2}\right)=g\left(\alpha_{1}^{0}, \alpha_{2}^{0}\right)>0,\left(\alpha_{1}, \alpha_{2}\right) \in D(g) \text { and }\left(\alpha_{1}^{0}, \alpha_{2}^{0}\right) \in D(g)
$$

In turn, Eqs.(B.1), (B.2) and (B.17) imply that, as long as $\left(\alpha_{1}^{0}, \alpha_{2}^{0}\right)$ is a fixed poin $\in D(g)$, it is impossible that $\gamma \rightarrow 0^{+}$as $\left(\alpha_{2}, \alpha_{2}\right) \rightarrow\left(\alpha_{1}^{0}, \alpha_{2}^{0}\right)$ with $\left(\alpha_{1}, \alpha_{2}\right) \in D(g)$. It becomes possible only if $\left(\alpha_{1}^{0}, \alpha_{2}^{0}\right)$ is replaced by some special fixed point that lies on the boundary of open domain $D(g)$ so that it lies outside of $D(g)$ and yet is a limit point of $D(g)$.

To search for all the special limit points referred to above, let (i)

$$
\bar{g}\left(\alpha_{1}, \alpha_{2}\right) \stackrel{\text { def }}{=} \sin ^{2} \alpha_{1}+\sin ^{2} \alpha_{2}+\sin ^{2}\left(\alpha_{1}+\alpha_{2}\right) \quad\left(\alpha_{1}, \alpha_{2}\right) \in D(\bar{g})
$$

where,

$$
D(\bar{g}) \stackrel{\text { def }}{=}\left\{\left(\alpha_{1}, \alpha_{2}\right) \mid \alpha_{1} \geq 0, \alpha_{2} \geq 0, \text { and } \alpha_{1}+\alpha_{2} \leq \pi\right\}
$$

and (iii) the line segments $L_{1}, L_{2}$ and $L_{3}$ depicted in Fig.11 be defined by

$$
\begin{aligned}
& L_{1} \stackrel{\text { def }}{=}\left\{\left(\alpha_{1}, \alpha_{2}\right) \mid 0 \leq \alpha_{1} \leq \pi \text { and } \alpha_{2}=0\right\} \\
& L_{2} \stackrel{\text { def }}{=}\left\{\left(\alpha_{1}, \alpha_{2}\right) \mid 0 \leq \alpha_{2} \leq \pi \text { and } \alpha_{1}=0\right\}
\end{aligned}
$$

and

$$
L_{3} \stackrel{\text { def }}{=}\left\{\left(\alpha_{1}, \alpha_{2}\right) \mid 0 \leq \alpha_{1} \leq \pi \text { and } \alpha_{1}+\alpha_{2}=\pi\right\}
$$

Then, (i) one can conclude from Eqs.(B.1), (B.2), (B.18) and (B.19) that the functions of $g$ and $\bar{g}$ are identical except that the domain $D(g)$ is only a proper subset of $D(\bar{g})$; (ii)

$$
D(\bar{g})=D(g) \cup(D(g))^{\prime}
$$

where $(D(g))^{\prime}$ denotes the boundary of $D(g)$, i.e., the set of all the limit points of the open domain $D(g)$ which lie outside of it, i.e.,

$$
D(g) \cap(D(g))^{\prime}=\varnothing
$$

where, $\varnothing$ denotes the empty set; (iii) By its definition Eq.(B.19), $D(\bar{g})$ contains all its own limit points and thus it is a closed domain [Ref.13, p.28]; and (iv) with the aid of Eqs.(B.20)-(B.22), the definition of $(D(g))^{\prime}$ and Fig.11, one concludes that

$$
(D(g))^{\prime}=L_{1} \cup L_{2} \cup L_{3}
$$

and

$$
L_{1} \cap L_{2}=\{(0,0)\}, L_{1} \cap L_{3}=\{(\pi, 0)\}, \text { and } L_{2} \cap L_{3}=\{(0, \pi)\}
$$


Next, let

$$
s(\alpha) \stackrel{\text { def }}{=} 2 \sin ^{2} \alpha, \quad 0 \leq \alpha \leq \pi
$$

Then, by using Eqs.(B.18)-(B.22), one has

$$
\begin{aligned}
& \bar{g}\left(\alpha_{1}, \alpha_{2}\right)=s\left(\alpha_{1}\right), 0 \leq \alpha_{1} \leq \pi \text { if }\left(\alpha_{1}, \alpha_{2}\right) \in L_{1} \\
& \bar{g}\left(\alpha_{1}, \alpha_{2}\right)=s\left(\alpha_{2}\right), 0 \leq \alpha_{2} \leq \pi \text { if }\left(\alpha_{1}, \alpha_{2}\right) \in L_{2}
\end{aligned}
$$

and

$$
\bar{g}\left(\alpha_{1}, \alpha_{2}\right)=s\left(\alpha_{1}\right), 0 \leq \alpha_{1} \leq \pi \text { if }\left(\alpha_{1}, \alpha_{2}\right) \in L_{3}
$$

On the other hand, Eq.(B.27) and the definitions

$$
\begin{gathered}
\dot{s}(\alpha) \stackrel{\text { def }}{=} \frac{d s}{d \alpha} \text { and } \ddot{s}(\alpha) \stackrel{\text { def }}{=} \frac{d^{2} s}{d \alpha^{2}}, 0<\alpha<\pi \\
\Rightarrow \quad \dot{s}(\alpha)=2 \sin (2 \alpha) \text { and } \ddot{s}(\alpha)=4 \cos (2 \alpha), 0<\alpha<\pi
\end{gathered}
$$

Eqs.(B.32), in turn, implies that

$$
\dot{s}(\alpha)=0 \Leftrightarrow \alpha=\pi / 2 \quad \text { if } 0<\alpha<\pi
$$

and

$$
\ddot{s}(\pi / 2)=-4<0
$$

By combining Eqs.(B.27), (B.33) and (B.34), one concludes that, (i) no local (relative) minimum of $s$ exists in the interval $0<\alpha<\pi$, (ii) the global maximal value of $s$ in the interval $0<\alpha<\pi$ is $s(\pi / 2)=2$, and it is attained if and only if $\alpha=\pi / 2$, i.e.,

$$
s(\pi / 2)=2>s(\alpha)>0 \text { if } 0<\alpha<\pi / 2 \text { or } \pi / 2<\alpha<\pi
$$

and (iii)

$$
s(0)=s(\pi)=0<s(\alpha), 0<\alpha<\pi
$$

With the aid of the results presented in the above items (i)-(iii), Eqs.(B.25) and (B.28)-Eqs.(B.30) imply that, over the boundary $(D(g))^{\prime}$ of the open domain $D(g)$, (i) the maximal value of $\bar{g}$ is 2 , and it is attained at and only at $\left(\alpha_{1}, \alpha_{2}\right)=(\pi / 2,0)$, or $\left(\alpha_{1}, \alpha_{2}\right)=(0, \pi / 2)$, or $\left(\alpha_{1}, \alpha_{2}\right)=(\pi / 2, \pi / 2)$; and (ii) the minimal value of $\bar{g}$ is $\mathbf{0}$, and it is attained at and only at $\left(\alpha_{1}, \alpha_{2}\right)=(0,0)$ or $\left(\alpha_{1}, \alpha_{2}\right)=$ $(\pi, 0)$ or $\left(\alpha_{1}, \alpha_{2}\right)=(0, \pi)$. Because (a) the function $\bar{g}$ is continuous over its domain $D(\bar{g})$, and (b) the points $(0,0),(\pi, 0)$ and $(0, \pi)$ on the $\alpha_{1}-\alpha_{2}$ plane are the only limit points of the open domain $D(g)$ which lie in $(D(g))^{\prime}$ and yet the function $\bar{g}$ attains its minimal value 0 at these points, Eq.(3.187) now follows directly from Eqs.(B.1), (B.2), (B.15)-(B.19) and (B.23). Note that the fact that the maximal value of $\bar{g}$ attained in $(D(g))^{\prime}$ is 2 , is consistent with Eq.(3.186).

\section{Acknowledgments}

The authors would like to acknowledge the support from Transformational Tools and Technologies (TTT) project under NASA's Transformative Aeronautics Concepts Program (TACP).

\section{References}

\footnotetext{
${ }^{1}$ Chang, S.-C., "The Method of Space-Time Conservation Element and Solution Element-A New Approach for Solving the Navier-Stokes and Euler Equations," J. Comput. Phys., Vol. 119, pp. 295-324, 1995.

${ }^{2}$ Chang, S., "A New Approach for Constructing Highly Stable High Order CESE Schemes," AIAA Paper 2010-543, 2010.

${ }^{3}$ Chang, C.-L., "Three-Dimensional Navier-Stokes Calculations Using the Modified Space-Time CESE Method," AIAA Paper 2007-5818, 2007.

${ }^{4}$ Chang, C.-L., Venkatachari, B., and Cheng, G, "Time-Accurate Local Time Stepping and High-Order Space-Time CESE Methods for Multi-Dimensional Flows with Unstructured Meshes," AIAA Paper 2013-3069, 2013.

${ }^{5}$ Chang, S.C., Wu, Y., Yang, V., and Wang, X.Y., "Local Time-Stepping Procedures for the Space-Time Conservation Element and Solution Element Method," Inter. J. of Comput. Fluid Dyn., Vol. 19, No. 5, pp. 359-380, 2005.
} 
${ }^{6}$ Yen, J. C., "Demonstration of a Multi-Dimensional Time-Accurate Local Time Stepping CESE Method," AIAA Paper 2011-2755, 2011.

${ }^{7}$ Candler, G., Barnhardt, M., Drayna, T., Nompelis, I., Peterson, D., and Subbareddy, P., "Unstructured Grid Approaches for Accurate Aeroheating Simulations," AIAA Paper 2007-3959, 2007.

${ }^{8}$ Nobel, B., and Daniel,J.W., Applied Linear Algebra, Prentice Hall Inc, 1977.

${ }^{9}$ Strang, G., Linear Algebra and Its Applications, Academic Press Inc, 1980.

10 “Turbulence Modeling Resource," NASA Langley Research Center, URL: http://turbmodels.larc.nasa.gov/ [cited 1 May $2017]$.

${ }^{11}$ Harvey, J. K., Holden, M.S, and Wadhams, T.P., "Code Validation Study Of Laminar Shock/Boundary Layer And Shock/Shock Interactions In Hypersonic Flow Part A: Experimental Measurements," AIAA Paper 2001-1031, 2001.

${ }^{12}$ Apostol, T.M., Calculus, Vol. 2: Multi-Variable Calculus and Linear Algebra with Applications to Differential Equations and Probability, 2nd Edition, John Wiley and Sons, Inc., 1969.

${ }^{13}$ Rudin, W., Principles of Mathematical Analysis, 2nd Edition, McGraw-Hill Book Company, 1964. 